\title{
Catalytic Asymmetric Reductive Amination of Aldehydes via Dynamic Kinetic Resolution
}

\author{
Sebastian Hoffmann, Marcello Nicoletti, and Benjamin List \\ Max-Planck-Institut für Kohlenforschung, Kaiser-Wilhelm-Platz 1, \\ D-45470 Mülheim an der Ruhr, Germany \\ list@mpi-muelheim.mpg.de
}

\section{Supporting Information}

General: All reactions were carried out under argon atmosphere in oven dried glassware with magnetic stirring. Unless otherwise stated, all reagents were purchased from commercial suppliers and used without further purification. All solvents used in the reactions were distilled from appropriate drying agents prior to use. Benzene was deoxygenated before it was used in reactions. Analytical thin-layer chromatography (TLC) was performed on silica gel precoated glass plates $(0.25 \mathrm{~mm}$ thickness, 60F-254, E. Merck). Visualization was accomplished by irradiation with a UV light at $254 \mathrm{~nm}$. Flash chromatography was performed using silica gel $60(0.040-0.063 \mathrm{~mm})$ from Merck.

Proton and carbon NMR spectra were recorded on a Bruker AV-400 and DX-300 spectrometer in $\mathrm{CDCl}_{3}$. Proton chemical shifts are reported in $\mathrm{ppm}(\delta)$ relative to tetramethylsilane (TMS) with the solvent resonance employed as the internal standard $\left(\mathrm{CDCl}_{3}, \delta 7.26 \mathrm{ppm}\right)$. Data are reported as follows: chemical shift, multiplicity ( $\mathrm{s}=$ singlet, $\mathrm{d}$ $=$ doublet, $\mathrm{q}=$ quartet, $\mathrm{m}=$ multiplet $)$, coupling constants $(\mathrm{Hz})$ and integration. ${ }^{13} \mathrm{C}$ chemical shifts are reported in ppm from tetramethylsilane (TMS) with the solvent resonance as the internal standard $\left(\mathrm{CDCl}_{3}, \delta 77.0 \mathrm{ppm}\right)$. Mass spectra were obtained on a Finnigan MAT 8200 (70 eV), accurate mass determinations were done on a Bruker APEX III FT-MS (7 T magnet). The enantiomeric excesses were determined by HPLC analysis employing a chiral stationary phase column (Daicel Co. Chiralcel unless otherwise noted) specified in the individual experiment, by comparing the samples with the appropriate racemic mixtures.

Preparation of Aldehydes: Aldehydes 1a-n are known compounds and the spectroscopic data are in agreement with the literature. Aldehydes $\mathbf{1 a}, \mathbf{1 1}$ and $\mathbf{1 m}$ are commercially available. Other aldehydes can easily be prepared from the corresponding ketones via Darzen 
reaction and subsequent hydrolysis/decarboxylation $(\mathbf{1 b}, \mathbf{1 d}-\mathbf{1 h}, \mathbf{1 j})$ or by Wittig reaction with methoxymethyltriphenylphosphonium chloride and cleavage of the resulting enol ether with $\operatorname{HBr}(\mathbf{1 c}, \mathbf{1 i}) .{ }^{1,2}$ The aldehydes $\mathbf{1 k}$ and $\mathbf{1 n}$ were obtained by Dess Martin and PCC oxidation of the corresponding alcohols. ${ }^{3,4}$

Typical procedure for the preparation of aldehydes 1 via Darzen reaction and subsequent hydrolysis/decarboxylation, illustrated with aldehyde 1e:

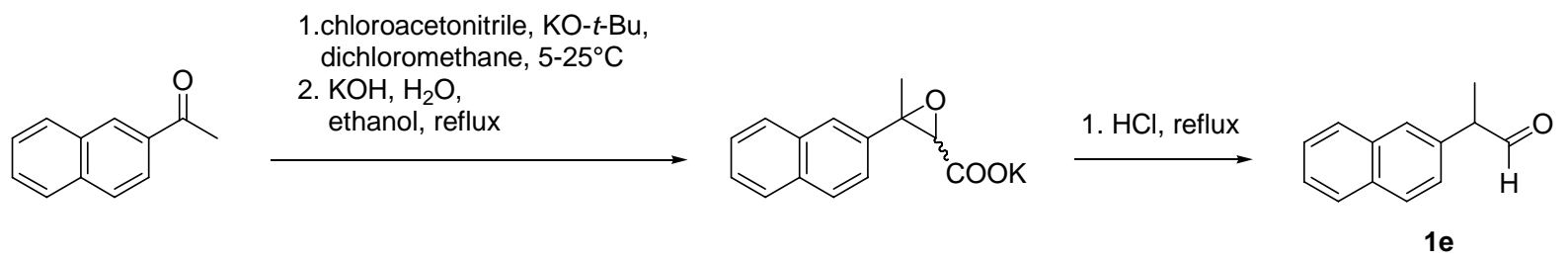

A mixture of $2^{2}$-Acetonaphtone $(5.95 \mathrm{~g}, 35.0 \mathrm{mmol})$ and chloroacetonitrile $(2.2 \mathrm{ml}, 35.0$ mmol) were dissolved in dichloromethane $(30 \mathrm{ml})$. A solution of potassium tert-butoxide in tert-butyl alcohol $(1.0 \mathrm{M}, 34.0 \mathrm{ml})$ was added dropwise under argon at $5{ }^{\circ} \mathrm{C}$ and stirred at 5-25 ${ }^{\circ} \mathrm{C}$ overnight. The reaction mixture was diluted with ice-cold water $(200 \mathrm{ml})$ and extracted with diethylether $(3 \times 50 \mathrm{ml})$. After washing the ethereal solution with ice-cold water $(2 \mathrm{x} 50$ $\mathrm{ml})$ and brine $(50 \mathrm{ml})$, the solution was dried over sodium sulfate. The solvent was removed under reduced pressure to yield the crude epoxy nitrile as a mixture of $(E)$ - and $(Z)$-isomers.

The crude epoxy nitrile was dissolved in ethanol $(46 \mathrm{ml})$ and 10\% potassium hydroxide solution $(34 \mathrm{ml})$ and refluxed for $15 \mathrm{~h}$. After evaporation to dryness, the residue was diluted with water $(50 \mathrm{ml})$ and washed with diethylether $(4 \mathrm{x} 50 \mathrm{ml})$. The aqueous solution was acidified with concentrated hydrochloric acid $(5 \mathrm{ml})$ and refluxed for $4 \mathrm{~h}$. After cooling to room temperature, the aqueous phase was extracted with diethylether $(3 \mathrm{x} 50 \mathrm{ml})$. The ethereal solution was washed with sodium bicarbonate, water, brine and dried over sodium sulphate, before removing the solvent. Distillation afforded aldehyde 1 e (1.5 g, 23\% yield).

\footnotetext{
${ }^{1}$ Svoboda, J.; Kocfeldova, Z.; Palecek, J. Collection Czechoslovak Chem. Commun. 1988, 53, 822-832.

${ }^{2}$ Danishefsky, S.; Harvey, D. F. J. Am. Chem. Soc. 1985, 107, 6647-6652.

${ }^{3}$ Stratakis, M.; Kalaitzakis, D.; Stavroulakis, D.; Kosmas, G.; Tsangarakis, C. Org. Lett. 2003, 5, 3471-3474.

${ }^{4}$ Wroblewski, A. E.; Piotrowska, D. G. Tetrahedron: Asymmetry 2002, 13, 2509-2512.
} 


\section{Typical procedure for the preparation of aldehydes 1 via Wittig reaction, illustrated}

with compound 1i:

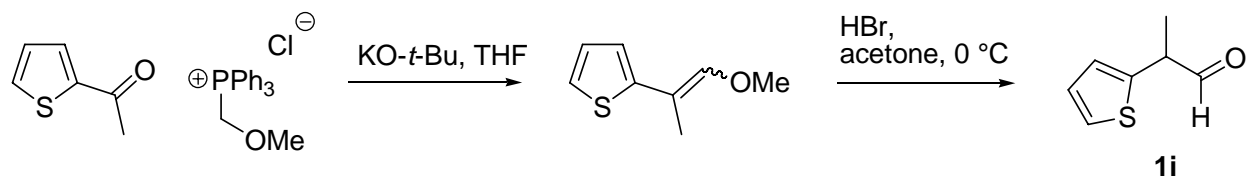

To a suspension of (methoxymethyl)-triphenylphosphonium chloride (25.9 g, $75.4 \mathrm{mmol})$ in dry THF $(130 \mathrm{ml})$ was added $\mathrm{KO}-\mathrm{t}-\mathrm{Bu}(8.1 \mathrm{~g}, 72.0 \mathrm{mmol})$ portion wise under an argon atmosphere at room temperature. The red suspension was stirred for 30 minutes at room temperature. A solution of 2-acetylthiophene $(5.84 \mathrm{~g}, 46.0 \mathrm{mmol})$ in THF $(100 \mathrm{ml})$ was then added dropwise, and the reaction mixture was stirred overnight. The solvent was removed under reduced pressure and hexane $(150 \mathrm{ml})$ was added to the residue. The resulting suspension was stirred for 30 minutes, and the $\mathrm{Ph}_{3} \mathrm{PO}$ was removed by filtration. After removing the solvent, the product was purified by distillation, yielding 2-(1-methoxyprop-1en-2-yl)thiophene $(5.7 \mathrm{~g}, 80 \%)$ as an E/Z-mixture: Colourless liquid; boiling point $120{ }^{\circ} \mathrm{C}, 10$ mbar; ${ }^{1} \mathrm{H}-\mathrm{NMR}\left(400 \mathrm{MHz}, \mathrm{CDCl}_{3}\right): \delta=1.99$ (s, 3H), 2.00 (s, 3H), 3.71 (s, 3H), 3.78 (s, 3H), $6.09(\mathrm{~s}, 1 \mathrm{H}), 6.57(\mathrm{~s}, 1 \mathrm{H}), 6.85(\mathrm{~m}, 1 \mathrm{H}), 6.94(\mathrm{~m}, 1 \mathrm{H}), 7.01(\mathrm{~m}, 2 \mathrm{H}), 7.07(\mathrm{~m}, 1 \mathrm{H}), 7.23(\mathrm{~m}$, $1 \mathrm{H}) ;{ }^{13} \mathrm{C}-\mathrm{NMR}\left(100 \mathrm{MHz}, \mathrm{CDCl}_{3}\right): \delta=13.0,17.3,60.0,107.3,109.9,121.0,121.1,122.9$, 124.1, 125.9, 127.1, 140.8, 143.3, 144.4; MS (EI):m/z (\%) = 154 (100), 139 (38), 126 (21), 111 (86); HRMS: Calculated for $\left[\mathrm{C}_{8} \mathrm{H}_{10} \mathrm{OS}\right]:$ 154.045237, found 154.045367.

To a solution of 2-(1-methoxyprop-1-en-2-yl)thiophene $(2.0 \mathrm{~g}, 13.0 \mathrm{mmol})$ in acetone $(12 \mathrm{ml})$ and water $(3 \mathrm{ml})$ was added concentrated $\mathrm{HBr}(48 \%, 0.45 \mathrm{ml})$ at $0{ }^{\circ} \mathrm{C}$. The reaction mixture was stirred for 2 days at room temperature. After neutralization with saturated $\mathrm{NaHCO}_{3}$ solution and extraction with diethylether $(3 \times 25 \mathrm{ml})$, the combined organic layers were dried over $\mathrm{Na}_{2} \mathrm{SO}_{4}$ and the solvent was removed under reduced pressure. The residue was purified by vacuum distillation, to give 2-(thiophen-2-yl)propanal ( $0.8 \mathrm{~g}, 5.7 \mathrm{mmol})$.

\section{1-Methoxy-2-(4-methoxyphenyl)propene:}

${ }^{1} \mathrm{H}-\mathrm{NMR}\left(300 \mathrm{MHz}, \mathrm{CDCl}_{3}\right)$ : $(E)$-isomer; $\delta=1.97$ (d, $\left.J=1.4 \mathrm{~Hz}, 3 \mathrm{H}\right), 3.70(\mathrm{~s}, 3 \mathrm{H}), 3.80$ (s, $3 \mathrm{H}), 6.32(\mathrm{q}, J=1.4 \mathrm{~Hz}, 1 \mathrm{H}), 6.83-6.89(\mathrm{~m}, 2 \mathrm{H}), 7.22-7.26(\mathrm{~m}, 2 \mathrm{H})$. (Z)-isomer; $\delta=1.90(\mathrm{~d}, J$ $=1.4 \mathrm{~Hz}, 2 \mathrm{H}), 3.66(\mathrm{~s}, 1 \mathrm{H}), 3.81(\mathrm{~s}, 3 \mathrm{H}), 6.06(\mathrm{q}, J=1.4 \mathrm{~Hz}, 1 \mathrm{H}), 6.83-6.89(\mathrm{~m}, 2 \mathrm{H}), 7.55-$ $7.58(\mathrm{~m}, 2 \mathrm{H}) .{ }^{13} \mathrm{C}-\mathrm{NMR}\left(75.5 \mathrm{MHz}, \mathrm{CDCl}_{3}\right):(E)$-isomer; $\delta=12.7,55.2,59.8,113.8,114.1$, 126.1, 133.2, 144.0, 158.0. (Z)-isomer; $\delta=18.3,55.2,60.0,110.4,113.3,128.6,131.0,143.5$, 
157.7. MS (EI): $\mathrm{m} / \mathrm{z}(\%)=178(100), 163$ (59), 135 (56); HRMS: Calculated for $\left[\mathrm{C}_{11} \mathrm{H}_{14} \mathrm{O}_{2}\right]$ : 178.099378, found 178.099317. The analytical data were consistent with the literature. ${ }^{5}$

2-(4-Methylphenyl)-propanal (1b): Colourless liquid; boiling point $110{ }^{\circ} \mathrm{C}, 10 \mathrm{mbar}$; ${ }^{1} \mathrm{H}$ $\operatorname{NMR}\left(400 \mathrm{MHz}, \mathrm{CDCl}_{3}\right): \delta=1.45(\mathrm{~d}, J=7.1 \mathrm{~Hz}, 3 \mathrm{H}), 2.38(\mathrm{~s}, 3 \mathrm{H}), 3.62$ (q, $\left.J=7.1 \mathrm{~Hz}, 1 \mathrm{H}\right)$, $7.13(\mathrm{~d}, J=8.0 \mathrm{~Hz}, 2 \mathrm{H}), 7.22(\mathrm{~d}, J=8.0 \mathrm{~Hz}, 2 \mathrm{H}), 9.69(\mathrm{~s}, 1 \mathrm{H}) ;{ }^{13} \mathrm{C}-\mathrm{NMR}(100 \mathrm{MHz}$, $\left.\mathrm{CDCl}_{3}\right): \delta=14.6,21.0,52.6,128.2,129.8,134.6,137.2,201.2 ; \mathrm{MS}(\mathrm{EI}): \mathrm{m} / \mathrm{z}(\%)=148(16)$, 119 (100), 103 (5), 91 (17), 77 (6); HRMS: Calculated for $\left[\mathrm{C}_{10} \mathrm{H}_{12} \mathrm{O}\right]$ : 148.088815, found 148.088968. The analytical data were consistent with the literature. ${ }^{1}$

2-(4-Methoxyphenyl)-propanal (1c): Colourless liquid; boiling point $120{ }^{\circ} \mathrm{C}, 10^{-1}$ mbar. ${ }^{1} \mathrm{H}$ $\operatorname{NMR}\left(300 \mathrm{MHz}, \mathrm{CDCl}_{3}\right): \delta=1.41(\mathrm{~d}, J=7.2 \mathrm{~Hz}, 3 \mathrm{H}), 3.58$ (q, $\left.J=7.2 \mathrm{~Hz}, 1 \mathrm{H}\right), 3.80(\mathrm{~s}, 3 \mathrm{H})$, $6.91(\mathrm{~m}, 2 \mathrm{H}), 7.12(\mathrm{~m}, 2 \mathrm{H}), 9.64(\mathrm{~s}, 1 \mathrm{H}) .{ }^{13} \mathrm{C}-\mathrm{NMR}\left(75.5 \mathrm{MHz}, \mathrm{CDCl}_{3}\right): \delta=14.6,52.1,55.2$, 114.5, 129.3, 129.6, 159.0, 201.1. MS (EI): m/z (\%) = 164 (14), 135 (100), 135 (100), 105 (16); HRMS: Calculated for $\left[\mathrm{C}_{10} \mathrm{H}_{12} \mathrm{O}_{2}\right]$ : 164.083734, found 164.083886. The analytical data were consistent with the literature. ${ }^{6}$

2-( $\alpha$-Naphthyl)-propionaldehyde (1d): white solid; boiling point $120{ }^{\circ} \mathrm{C}, 10^{-1} \mathrm{mbar} ;{ }^{1} \mathrm{H}$ $\operatorname{NMR}\left(400 \mathrm{MHz}, \mathrm{CDCl}_{3}\right): \delta=1.59(\mathrm{~d}, J=7.0 \mathrm{~Hz}, 3 \mathrm{H}), 4.38(\mathrm{q}, J=7.0 \mathrm{~Hz}, 1 \mathrm{H}), 7.28(\mathrm{~d}, J=$ $7.4 \mathrm{~Hz}, 1 \mathrm{H}), 7.53(\mathrm{~m}, 3 \mathrm{H}), 7.85(\mathrm{~d}, J=8.1 \mathrm{~Hz}, 1 \mathrm{H}), 7.90$ (d, $J=8.1 \mathrm{~Hz}, 1 \mathrm{H}), 8.02(\mathrm{~d}, J=8.4$ $\mathrm{Hz}, 1 \mathrm{H}), 9.77(\mathrm{~s}, 1 \mathrm{H}) ;{ }^{13} \mathrm{C}-\mathrm{NMR}\left(100 \mathrm{MHz}, \mathrm{CDCl}_{3}\right): \delta=14.6,48.8,122.9,125.6,125.6,125.9$, 126.6, 128.3, 129.1, 131.7, 134.1, 201.2; MS (EI):m/z (\%) = 184 (23), 155 (100), 141 (6), 128 (9), 115 (7); HRMS: Calculated for $\left[\mathrm{C}_{13} \mathrm{H}_{12} \mathrm{O}\right]$ : 184.088819, found 184.088636. The analytical data were consistent with the literature. ${ }^{7}$

2-( $\beta$-Naphthyl)-propionaldehyde (1e): colourless solid; boiling point $110{ }^{\circ} \mathrm{C}, 10^{-1} \mathrm{mbar} ;{ }^{1} \mathrm{H}$ $\operatorname{NMR}\left(400 \mathrm{MHz}, \mathrm{CDCl}_{3}\right): \delta=1.56(\mathrm{~d}, J=7.0 \mathrm{~Hz}, 3 \mathrm{H}), 3.81$ (q, $\left.J=7.0 \mathrm{~Hz}, 1 \mathrm{H}\right), 7.33(\mathrm{~d}, J=$ $8.6 \mathrm{~Hz}, 1 \mathrm{H}), 7.52(\mathrm{~m}, 2 \mathrm{H}), 7.70(\mathrm{~s}, 1 \mathrm{H}), 7.85(\mathrm{~m}, 3 \mathrm{H}), 9.78(\mathrm{~s}, 1 \mathrm{H}) ;{ }^{13} \mathrm{C}-\mathrm{NMR}(100 \mathrm{MHz}$, $\left.\mathrm{CDCl}_{3}\right): \delta=14.6,53.0,126.1,126.1,126.4,127.1,127.7,128.8,132.6,133.6,135.1,200.9$; MS (EI):m/z (\%) = 184 (24), 155 (100), 141 (4), 128 (8), 115 (6); HRMS: Calculated for

\footnotetext{
${ }^{5}$ Tokunaga, M; Shirogane, Y.; Aoyama, H.; Obora, Y.; Tsuji, Y. J. Organomet. Chem. 2005, 690, 5378-5382.

${ }^{6}$ Meyers, A. I.; Walkup, R. D. Tetrahedron 1985, 41, 5089-5106.

${ }^{7}$ Fieser, L. F.; Joshel, L. M.; Seligman, A. M. J. Am. Chem. Soc. 1939, 61, 2134-2139.
} 
$\left[\mathrm{C}_{13} \mathrm{H}_{12} \mathrm{O}\right]:$ : 184.088815, found 184.088891. The analytical data were consistent with the literature. $^{8}$

2-(4-Bromophenyl)-propanal (1f): Colourless liquid; boiling point $70{ }^{\circ} \mathrm{C}, 10^{-1} \mathrm{mbar} ;{ }^{1} \mathrm{H}$ NMR (400 MHz, $\left.\mathrm{CDCl}_{3}\right): \delta=1.43(\mathrm{~d}, J=7.1 \mathrm{~Hz}, 3 \mathrm{H}), 3.61(\mathrm{q}, J=7.1 \mathrm{~Hz}, 1 \mathrm{H}), 7.08(\mathrm{~d}, J=$ $8.4 \mathrm{~Hz}, 2 \mathrm{H}), 7.50(\mathrm{~d}, J=8.4 \mathrm{~Hz}, 2 \mathrm{H}), 9.66(\mathrm{~s}, 1 \mathrm{H}) ;{ }^{13} \mathrm{C}-\mathrm{NMR}\left(100 \mathrm{MHz}, \mathrm{CDCl}_{3}\right): \delta=14.5$, 52.3, 121.6, 129.9, 132.2, 136.7, 200.3; MS (EI):m/z (\%) = 214 (18), 212 (18), 185 (88), 183 (91), 104 (100), 103 (25), 77 (22); HRMS: Calculated for [ $\mathrm{C}_{9} \mathrm{H}_{9} \mathrm{BrO}$ ]: 211.983691, found 211.983943. The analytical data were consistent with the literature. ${ }^{1}$

2-(2-Fluorophenyl)-propanal (1g): Colourless liquid; boiling point $90{ }^{\circ} \mathrm{C}, 10 \mathrm{mbar} ;{ }^{1} \mathrm{H}$ NMR (400 MHz, $\left.\mathrm{CDCl}_{3}\right): \delta=1.48(\mathrm{~d}, J=7.2 \mathrm{~Hz}, 3 \mathrm{H}), 3.93(\mathrm{q}, J=7.2 \mathrm{~Hz}, 1 \mathrm{H}), 7.21(\mathrm{~m}, 4 \mathrm{H})$, $9.76(\mathrm{~s}, 1 \mathrm{H}) ;{ }^{13} \mathrm{C}-\mathrm{NMR}\left(100 \mathrm{MHz}, \mathrm{CDCl}_{3}\right): \delta=13.7,46.4,115.7\left(\mathrm{~d}, J_{\mathrm{CF}}=21 \mathrm{~Hz}\right), 124.6(\mathrm{~d}$, $\left.J_{\mathrm{CF}}=3 \mathrm{~Hz}\right), 125.3\left(\mathrm{~d}, J_{\mathrm{CF}}=15 \mathrm{~Hz}\right), 129.2\left(\mathrm{~d}, J_{\mathrm{CF}}=8 \mathrm{~Hz}\right), 129.4\left(\mathrm{~d}, J_{\mathrm{CF}}=4 \mathrm{~Hz}\right), 160.8\left(\mathrm{~d}, J_{\mathrm{CF}}\right.$ = $246 \mathrm{~Hz}), 200.1 ; \mathrm{MS}(\mathrm{EI}): \mathrm{m} / \mathrm{z}(\%)=152$ (22), 124 (10), 123 (100), 109 (10), 103 (47), 97 (6), 77 (15); HRMS: Calculated for [ $\left.\mathrm{C}_{9} \mathrm{H}_{9} \mathrm{FO}\right]$ : 152.063741, found 152.063772. The analytical data were consistent with the literature. ${ }^{9}$

2-(3-Fluorophenyl)-propanal (1h): Colourless liquid; boiling point $100{ }^{\circ} \mathrm{C}, 10 \mathrm{mbar} ;{ }^{1} \mathrm{H}$ NMR (400 MHz, $\left.\mathrm{CDCl}_{3}\right): \delta=1.45(\mathrm{~d}, J=7.1 \mathrm{~Hz}, 3 \mathrm{H}), 3.64$ (q, $\left.J=7.1 \mathrm{~Hz}, 1 \mathrm{H}\right), 6.97(\mathrm{~m}, 3 \mathrm{H})$, $7.30(\mathrm{~m}, 1 \mathrm{H}), 9.67(\mathrm{~s}, 1 \mathrm{H}) ;{ }^{13} \mathrm{C}-\mathrm{NMR}\left(100 \mathrm{MHz}, \mathrm{CDCl}_{3}\right): \delta=14.5,52.6,114.5\left(\mathrm{~d}, J_{\mathrm{CF}}=21\right.$ $\mathrm{Hz}), 115.2\left(\mathrm{~d}, J_{\mathrm{CF}}=21 \mathrm{~Hz}\right), 123.9\left(\mathrm{~d}, J_{\mathrm{CF}}=3 \mathrm{~Hz}\right), 130.5\left(\mathrm{~d}, J_{\mathrm{CF}}=8 \mathrm{~Hz}\right), 140.1\left(\mathrm{~d}, J_{\mathrm{CF}}=7\right.$ $\mathrm{Hz}), 163.2\left(\mathrm{~d}, J_{\mathrm{CF}}=248 \mathrm{~Hz}\right), 200.3$; MS (EI):m/z (\%) = 152 (26), 124 (17), 123 (100), 109 (17), 103 (49), 97 (9), 77 (15); HRMS: Calculated for [ $\left.\mathrm{C}_{9} \mathrm{H}_{9} \mathrm{FO}\right]$ : 152.063742, found 152.063696. The analytical data were consistent with the literature. ${ }^{10}$

2-(2-Thiophenyl)-propanal (1i): Colourless liquid; boiling point $120{ }^{\circ} \mathrm{C}, 10 \mathrm{mbar}$; ${ }^{1} \mathrm{H}-\mathrm{NMR}$ $\left(400 \mathrm{MHz} \mathrm{CDCl}_{3}\right): \delta=1.52(\mathrm{~d}, J=7.1 \mathrm{~Hz}, 3 \mathrm{H}), 3.88(\mathrm{q}, J=7.1 \mathrm{~Hz}, 1 \mathrm{H}), 6.93(\mathrm{~d}, J=3.5 \mathrm{~Hz}$, 1H), $7.04(\mathrm{dd}, J=3.5$ and $5.1 \mathrm{~Hz}, 1 \mathrm{H}), 7.28(\mathrm{~d}, J=5.1 \mathrm{~Hz}, 1 \mathrm{H}), 9.64(\mathrm{~s}, 1 \mathrm{H}) ;{ }^{13} \mathrm{C}-\mathrm{NMR}(100$ $\left.\mathrm{MHz}, \mathrm{CDCl}_{3}\right): \delta=15.3,47.8,125.1,125.4,127.4,140.1,199.1 ; \mathrm{MS}(\mathrm{EI}): \mathrm{m} / \mathrm{z}(\%)=140(17)$,

\footnotetext{
${ }^{8}$ Krasodomski, W.; Luczynski, M. K.; Wilamowski, J.; Sepiol, J. J. Tetrahedron 2003, 59, 5677-5683.

${ }^{9}$ Nozaki, K.; Sakai, N.; Nanno, T.; Horiuchi, T.; Takaya, H. J. Am. Chem. Soc. 1997, 119, 4413-4423.

${ }^{10}$ Wang, L. L.; Guo, R. W.; Li, Y. M.; Chan, A. S. C. Tetrahedron: Asymmetry 2005, 16, 3198-3204.
} 
111 (100), 97 (4), 85 (5), 77 (14); HRMS: Calculated for $\left[\mathrm{C}_{7} \mathrm{H}_{8} \mathrm{OS}\right]$ : 140.029591, found 140.029675. The analytical data were consistent with the literature. ${ }^{11}$

2-(Cyclohexyl)-propanal (1j): Colourless liquid; boiling point $83{ }^{\circ} \mathrm{C}, 40$ mbar. ${ }^{1} \mathrm{H}-\mathrm{NMR}$ $\left(300 \mathrm{MHz}, \mathrm{CDCl}_{3}\right): \delta=1.04(\mathrm{~d}, J=6.8 \mathrm{~Hz}, 3 \mathrm{H}), 1.10-1.32(\mathrm{~m}, 5 \mathrm{H}), 1.58-1.78(\mathrm{~m}, 6 \mathrm{H}), 2.17-$ $2.24(\mathrm{~m}, 1 \mathrm{H}), 9.64(\mathrm{~d}, J=2.3 \mathrm{~Hz}, 1 \mathrm{H}) .{ }^{13} \mathrm{C}-\mathrm{NMR}\left(75.5 \mathrm{MHz}, \mathrm{CDCl}_{3}\right): \delta=9.6,26.2,26.3$, $26.4,38.5,51.8,205.8$. The analytical data were consistent with the literature. ${ }^{6}$

2-(tert.Butyl)-propanal (1k): Methyl 3,3-dimethylbutanoate (4.02 g, $30.8 \mathrm{mmol})$ was added dropwise to a solution of LDA in THF $(50 \mathrm{ml})$ at $-78^{\circ} \mathrm{C}$ and the solution was stirred for 1 hour. MeI (3.82 ml, $61.4 \mathrm{mmol})$ was then added dropwise (1 hour) and the mixture stirred at $78{ }^{\circ} \mathrm{C}$ for 2 hours and 2 hours at room temperature. The mixture was then quenched with water $(40 \mathrm{ml})$ and extracted with diethylether $(3 \times 100 \mathrm{ml})$. The organic layers were washed with $\mathrm{NH}_{4} \mathrm{Cl}$ (saturated solution), brine and water, dried $\left(\mathrm{Na}_{2} \mathrm{SO}_{4}\right)$ and evaporated to yield Methyl 2,3,3-trimethylbutanoate $(3.89 \mathrm{~g}, 89 \%)$ as a clear oil. Part of this material $(2.10 \mathrm{~g}$, $14.5 \mathrm{mmol})$ in THF $(20 \mathrm{ml})$ was added dropwise to a suspension of $\mathrm{LiAlH}_{4}(0.60 \mathrm{~g}, 15.9$ mmol) in THF $(60 \mathrm{ml})$ at $0{ }^{\circ} \mathrm{C}$. The reaction mixture was stirred at room temperature overnight and after workup, gave pure 2,3,3-trimethylbutanol (1.2 g, 71\%) as a clear oil.

Dess-Martin periodinane $(8.9 \mathrm{~g}, 21.0 \mathrm{mmol})$ was added portionwise to a solution of 2,3,3trimethylbutanol $(1.2,10.3 \mathrm{mmol})$ in DCM $(60 \mathrm{ml}) .^{12}$ The suspension was stirred at room temperature for 1 hour and the reaction monitored by GC/MS. After disappearance of the starting alcohol, diethylether $(30 \mathrm{ml})$ was added followed by a solution of sodium thiosulphate $(20 \mathrm{~g})$ in $80 \% \mathrm{NaHCO}_{3}$ saturated solution $(40 \mathrm{ml})$. The organic layers were separated and the aqueous phase was extracted with diethylether $(2 \mathrm{x} 40 \mathrm{ml})$. The combined organic solution were washed with $\mathrm{NaHCO}_{3}$, water and brine. After drying over $\mathrm{Na}_{2} \mathrm{SO}_{4}$, the solvent was evaporated to yield the product $(0.75 \mathrm{~g}, 64 \%)$ as a clear oil. ${ }^{1} \mathrm{H}-\mathrm{NMR}\left(400 \mathrm{MHz}, \mathrm{CDCl}_{3}\right): \delta=$ $1.02(\mathrm{~s}, 9 \mathrm{H}), 1.05(\mathrm{~d}, J=7.2 \mathrm{~Hz}, 3 \mathrm{H}), 2.15(\mathrm{~m}, 1 \mathrm{H}), 9.78(\mathrm{~s}, 1 \mathrm{H})$; The analytical data were consistent with the literature. ${ }^{6}$

2-Phenylbutyraldehyde (1n): 2-Phenylbutyraldehyde 1n was prepared starting from 2phenylbutanol. A solution of 2-phenylbutanol $(10.0 \mathrm{~g}, 67.0 \mathrm{mmol})$ in dichloromethane $(70 \mathrm{ml})$ was added to a suspension of pyridiniumchlorochromate $(25.0 \mathrm{~g}, 116.0 \mathrm{mmol})$ in dichloromethane $(70 \mathrm{ml})$ at $0{ }^{\circ} \mathrm{C}$. After complete conversion of the alcohol, the mixture was

\footnotetext{
${ }^{11}$ Tamaru, Y.; Yamada, Y.; Yoshida, Z. I. Tetrahedron 1979, 35, 329-340.

${ }^{12}$ Wroblewski, A. E.; Piotrowska, D. G. Tetrahedron: Asymmetry 2002, 13, 2509-2512.
} 
filtered over silica. The solvent was removed, and the remaining oil distilled under vacuum (12 mbar, $\left.105{ }^{\circ} \mathrm{C}\right)$ to yield 2-Phenylbutyraldehyde $(6.0 \mathrm{~g}, 60 \%)$. The analytical data were consistent with the literature. ${ }^{13,14}$

Colourless liquid; ${ }^{1} \mathrm{H}-\mathrm{NMR}\left(400 \mathrm{MHz}, \mathrm{CDCl}_{3}\right): \delta=0.91(\mathrm{t}, J=7.4 \mathrm{~Hz}, 3 \mathrm{H}), 1.76(\mathrm{~m}, 1 \mathrm{H})$, $2.12(\mathrm{~m}, 1 \mathrm{H}), 3.41(\mathrm{~m}, 1 \mathrm{H}), 7.29(\mathrm{~m}, 5 \mathrm{H}), 9.68(\mathrm{~d}, J=2.0 \mathrm{~Hz}, 1 \mathrm{H}) ;{ }^{13} \mathrm{C}-\mathrm{NMR}(100 \mathrm{MHz}$, $\left.\mathrm{CDCl}_{3}\right): \delta=11.7,22.9,60.8,127.5,128.8,129.0,136.3,201.0 ; \mathrm{MS}(\mathrm{EI}): \mathrm{m} / \mathrm{z}(\%)=148(17)$, 119 (45), 91 (100), 77 (7); HRMS: Calculated for $\left[\mathrm{C}_{10} \mathrm{H}_{12} \mathrm{O}\right]:$ 148.088814, found 148.088662.

\section{Dihydropyridine Screening:}
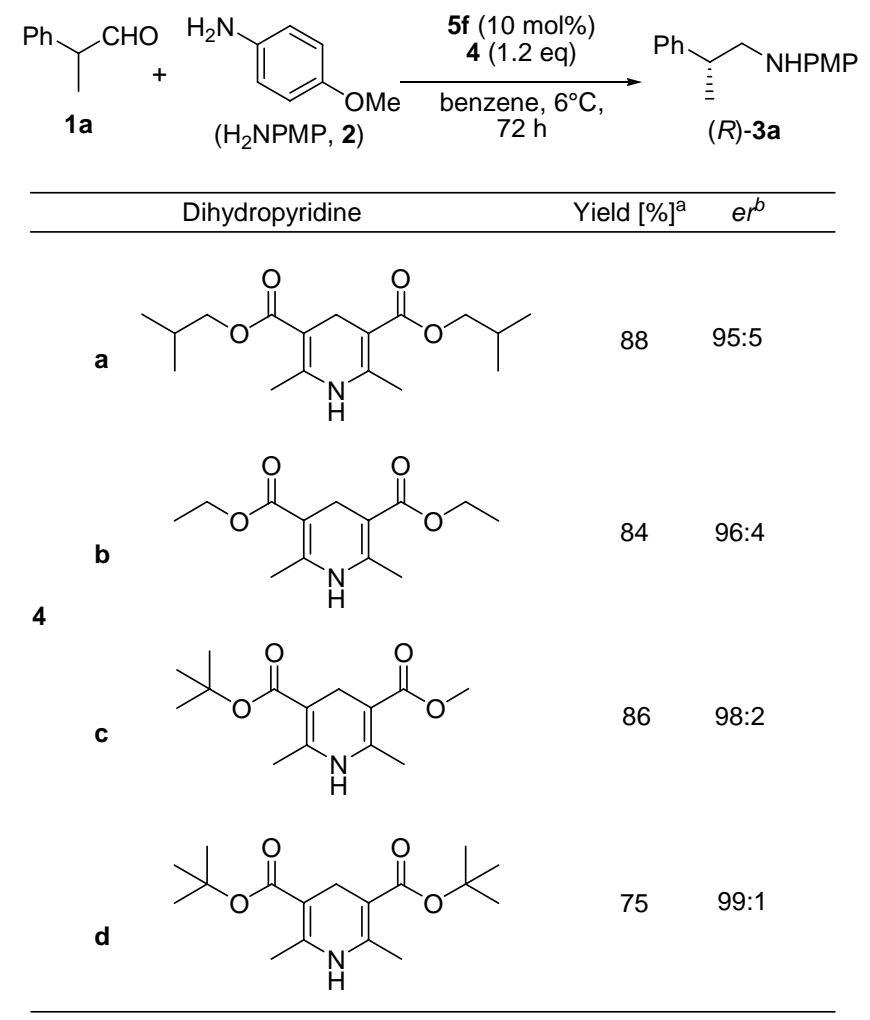

a Determined by GC with internal standard. ${ }^{\mathrm{b}}$ er determined by HPLC.

\section{General procedure for the asymmetric reductive amination:}

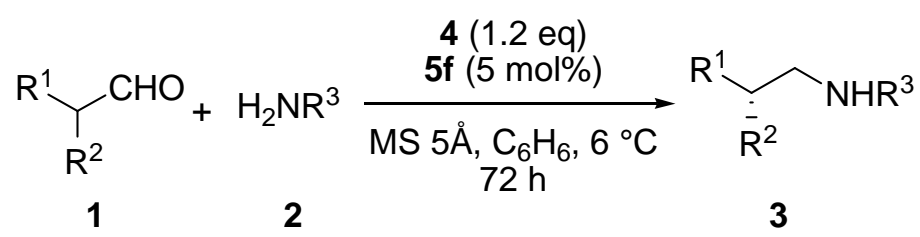

A mixture of amine 2 (0.5 mmol), Hantzsch ester 4 (160 mg, $0.6 \mathrm{mmol}, 1.2 \mathrm{eq})$, phosphoric acid $\mathbf{5 f}(19 \mathrm{mg}, 0.025 \mathrm{mmol})$, freshly activated molecular sieve $5 \AA$ (350 mg) and benzene (10 $\mathrm{mL}$ ) was cooled to $6{ }^{\circ} \mathrm{C}$ under an argon atmosphere. Aldehyde 1 was added and the reaction mixture was stirred for 3 days at $6{ }^{\circ} \mathrm{C}$. The solvent was evaporated at reduced pressure and the

\footnotetext{
${ }^{13}$ Stratakis, M.; Kalaitzakis, D.; Stavroulakis, D.; Kosmas, G.; Tsangarakis, C. Org. Lett. 2003, 5, 3471-3474.

${ }^{14}$ Lodge, E. P.; Heathcock, C. H. J. Am. Chem. Soc. 1987, 109, 3353-3361.
} 
products were isolated by flash chromatography $\left(\mathrm{SiO}_{2}\right.$, ethyl acetate/hexane) to give pure amines 3. The yields and enantiomeric excesses are given in table 2.

Amine 3a: purification by flash chromatography (5\% EtOAc in hexanes). ${ }^{1} \mathrm{H}-\mathrm{NMR}$ (400 $\left.\mathrm{MHz}, \mathrm{CDCl}_{3}\right): \delta=1.26(\mathrm{~d}, J=7.2 \mathrm{~Hz}, 3 \mathrm{H}), 2.97(\mathrm{~m}, 1 \mathrm{H}), 3.11(\mathrm{dd}, J=8.3$ and $12.2 \mathrm{~Hz}, 1 \mathrm{H})$, $3.23(\mathrm{dd}, J=6.0$ and $12.2 \mathrm{~Hz}, 1 \mathrm{H}), 3.67(\mathrm{~s}, 3 \mathrm{H}), 6.47(\mathrm{~d}, J=9.0 \mathrm{~Hz}, 2 \mathrm{H}), 6.69$ (d, $J=9.0 \mathrm{~Hz}$, 2H), $7.16(\mathrm{~m}, 3 \mathrm{H}), 7.24(\mathrm{~m}, 1 \mathrm{H}) ;{ }^{13} \mathrm{C}-\mathrm{NMR}\left(100 \mathrm{MHz}, \mathrm{CDCl}_{3}\right): \delta=19.8,39.2,52.0,55.8$, 114.4, 114.9, 126.6, 127.2, 128.6, 142.2, 144.6, 152.1; MS (EI):m/z (\%) = 241 (14), 136 (100), 121 (5), 105 (4); HRMS: Calculated for [ $\mathrm{C}_{16} \mathrm{H}_{19} \mathrm{NO}$ ]: 241.146664, found 241.146527. The enantiomers were analyzed by chiral HPLC using a OJ-H column (n-heptane/2-Propanol $=90 / 10$, flow rate $=0.5 \mathrm{ml} / \mathrm{min}$, wavelength $=220 \mathrm{~nm}$, major enantiomer: $t_{\mathrm{R}}=32.43 \mathrm{~min}$, minor enantiomer: $t_{R}=29.20 \mathrm{~min}$ ).

Amine 3b: purification by flash chromatography (25\% dichloro-methane in hexane). ${ }^{1} \mathrm{H}-$ NMR (400 MHz, $\left.\mathrm{CDCl}_{3}\right): \delta=1.32(\mathrm{~d}, J=7.0 \mathrm{~Hz}, 3 \mathrm{H}), 2.35(\mathrm{~s}, 3 \mathrm{H}), 3.03(\mathrm{~m}, 1 \mathrm{H}), 3.17$ (dd, $J$ $=8.3$ and $12.1 \mathrm{~Hz}, 1 \mathrm{H}), 3.29(\mathrm{dd}, J=6.1$ and $12.1 \mathrm{~Hz}, 1 \mathrm{H}), 3.75(\mathrm{~s}, 3 \mathrm{H}), 6.56(\mathrm{~d}, J=8.9 \mathrm{~Hz}$, 2H), $6.78(\mathrm{~d}, J=9.0 \mathrm{~Hz}, 2 \mathrm{H}), 7.13(\mathrm{~m}, 4 \mathrm{H}) ;{ }^{13} \mathrm{C}-\mathrm{NMR}\left(100 \mathrm{MHz}, \mathrm{CDCl}_{3}\right): \delta=19.9,21.0$, $38.8,52.1,55.8,114.5,114.9,127.1,129.3,136.1,141.5,142.2,152.2 ; \mathrm{MS}(\mathrm{EI}): \mathrm{m} / \mathrm{z}(\%)=$ 255 (13), 136 (100), 119 (5), 108 (4); HRMS: Calculated for $\left[\mathrm{C}_{17} \mathrm{H}_{21} \mathrm{NONa}\right]: 278.151534$, found 278.151671. The enantiomers were analyzed by chiral HPLC using a AS-H column (nheptane/2-Propanol $=99.5 / 0.5$, flow rate $=0.5 \mathrm{ml} / \mathrm{min}$, wavelength $=220 \mathrm{~nm}$, major enantiomer: $t_{R}=45.42 \mathrm{~min}$, minor enantiomer: $t_{R}=60.67 \mathrm{~min}$ ).

Amine 3c: purification by flash chromatography (dichloro-methane). ${ }^{1} \mathrm{H}-\mathrm{NMR}(400 \mathrm{MHz}$, $\left.\mathrm{CDCl}_{3}\right): \delta=1.30(\mathrm{~d}, J=6.7 \mathrm{~Hz}, 3 \mathrm{H}), 3.00(\mathrm{~m}, 1 \mathrm{H}), 3.14(\mathrm{dd}, J=8.5$ and $12.2 \mathrm{~Hz}, 1 \mathrm{H}), 3.29$ $(\mathrm{dd}, J=6.0$ and $12.2 \mathrm{~Hz}, 1 \mathrm{H}), 3.74(\mathrm{~s}, 3 \mathrm{H}), 3.80(\mathrm{~s}, 3 \mathrm{H}), 6.57(\mathrm{~d}, J=8.9 \mathrm{~Hz}, 2 \mathrm{H}), 6.77$ (d, $J=$ $8.9 \mathrm{~Hz}, 2 \mathrm{H}), 6.87(\mathrm{~d}, J=8.7 \mathrm{~Hz}, 2 \mathrm{H}), 7.14(\mathrm{~d}, J=8.7 \mathrm{~Hz}, 2 \mathrm{H}) ;{ }^{13} \mathrm{C}-\mathrm{NMR}\left(100 \mathrm{MHz}, \mathrm{CDCl}_{3}\right)$ : $\delta=20.0,38.3,52.4,55.3,55.8,114.1,114.7,114.9,128.1,136.5,141.9,152.3,158.3$; MS (EI):m/z (\%) = 271 (17), 136 (100), 121 (4), 108 (2); HRMS: Calculated for $\left[\mathrm{C}_{17} \mathrm{H}_{21} \mathrm{NO}_{2}\right]$ : 271.157230, found 271.157009. The enantiomers were analyzed by chiral HPLC using a ADH column (n-heptane $/ 2$-Propanol $=98 / 2$, flow rate $=0.5 \mathrm{ml} / \mathrm{min}$, wavelength $=220 \mathrm{~nm}$, major enantiomer: $t_{R}=18.30$ min, minor enantiomer: $t_{R}=19.56 \mathrm{~min}$ ). 
Amine 3d: purification by flash chromatography (25\% dichloro-methane in hexane). ${ }^{1} \mathrm{H}-$ NMR (400 MHz, $\left.\mathrm{CDCl}_{3}\right): \delta=1.53(\mathrm{~d}, J=6.9 \mathrm{~Hz}, 3 \mathrm{H}), 3.47(\mathrm{dd}, J=6.6$ and $12.3 \mathrm{~Hz}, 1 \mathrm{H})$, $3.55(\mathrm{dd}, J=7.2$ and $12.1 \mathrm{~Hz}, 1 \mathrm{H}), 3.80(\mathrm{~s}, 3 \mathrm{H}), 4.02(\mathrm{~m}, 1 \mathrm{H}), 6.60(\mathrm{~d}, J=8.9 \mathrm{~Hz}, 2 \mathrm{H}), 6.83$ $(\mathrm{d}, J=8.9 \mathrm{~Hz}, 2 \mathrm{H}), 7.51(\mathrm{~m}, 4 \mathrm{H}), 7.82$ (d, $J=7.9 \mathrm{~Hz}, 1 \mathrm{H}), 7.95(\mathrm{~d}, J=9.5 \mathrm{~Hz}, 1 \mathrm{H}), 8.18(\mathrm{~d}, J$ $=9.4 \mathrm{~Hz}, 1 \mathrm{H}) ;{ }^{13} \mathrm{C}-\mathrm{NMR}\left(100 \mathrm{MHz}, \mathrm{CDCl}_{3}\right): \delta=19.9,33.5,51.4,55.7,114.2,114.8,122.7$, 123.0, 125.5, 125.6, 125.9, 126.9, 128.9, 131.8, 134.0, 140.6, 142.4, 152.1; MS (EI):m/z (\%) = 291 (13), 155 (3), 136 (100), 121 (4), 108 (3); HRMS: Calculated for $\left[\mathrm{C}_{20} \mathrm{H}_{21} \mathrm{NONa}\right]$ : 314.151529, found 314.151079. The enantiomers were analyzed by chiral HPLC using a OD$\mathrm{H}$ column (n-heptane/2-Propanol $=70 / 30$, flow rate $=0.5 \mathrm{ml} / \mathrm{min}$, wavelength $=220 \mathrm{~nm}$, major enantiomer: $t_{R}=20.92 \min$, minor enantiomer: $t_{R}=16.23 \mathrm{~min}$ ).

Amine 3e: purification by flash chromatography (3\% EtOAc in hexane). ${ }^{1} \mathrm{H}-\mathrm{NMR}(400 \mathrm{MHz}$, $\left.\mathrm{CDCl}_{3}\right): \delta=1.43(\mathrm{~d}, J=6.8 \mathrm{~Hz}, 3 \mathrm{H}), 3.23(\mathrm{~m}, 1 \mathrm{H}), 3.32(\mathrm{dd}, J=8.2$ and $12.0 \mathrm{~Hz}, 1 \mathrm{H}), 3.41$ $(\mathrm{dd}, J=5.8$ and $12.0 \mathrm{~Hz}, 1 \mathrm{H}), 3.75(\mathrm{~s}, 3 \mathrm{H}), 6.56(\mathrm{~d}, J=8.9 \mathrm{~Hz}, 2 \mathrm{H}), 6.78(\mathrm{~d}, J=8.9 \mathrm{~Hz}, 2 \mathrm{H})$, $7.39(\mathrm{~d}, J=10.2 \mathrm{~Hz}, 1 \mathrm{H}), 7.48(\mathrm{~m}, 2 \mathrm{H}), 7.67(\mathrm{~s}, 1 \mathrm{H}), 7.83(\mathrm{~m}, 3 \mathrm{H}) ;{ }^{13} \mathrm{C}-\mathrm{NMR}(100 \mathrm{MHz}$, $\left.\mathrm{CDCl}_{3}\right): \delta=19.9,39.4,51.9,55.8,114.5,114.9,125.5,125.9,126.1,127.6,127.6,128.4$, 132.5, 133.6, 142.0, 142.1, 152.2; MS (EI):m/z (\%) = 291 (14), 155 (6), 136 (100), 121 (5); HRMS: Calculated for $\left[\mathrm{C}_{20} \mathrm{H}_{22} \mathrm{NO}\right]: 292.169590$ found 292.169781. The enantiomers were analyzed by chiral HPLC using a OD-H column (n-heptane/2-Propanol = 90/10, flow rate $=$ $0.5 \mathrm{ml} / \mathrm{min}$, wavelength $=254 \mathrm{~nm}$, major enantiomer: $t_{R}=16.61 \mathrm{~min}$, minor enantiomer: $t_{R}=$ $17.92 \mathrm{~min})$.

Amine 3f: purification by flash chromatography (30\% dichloro-methane in hexane). ${ }^{1} \mathrm{H}-\mathrm{NMR}$ $\left(400 \mathrm{MHz}, \mathrm{CDCl}_{3}\right): \delta=1.30(\mathrm{~d}, J=7.1 \mathrm{~Hz}, 3 \mathrm{H}), 3.02(\mathrm{~m}, 1 \mathrm{H}), 3.18(\mathrm{dd}, J=8.3$ and $12.5 \mathrm{~Hz}$, 1H), $3.30(\mathrm{dd}, J=6.0$ and $12.5 \mathrm{~Hz}, 1 \mathrm{H}), 3.75$ (s, 3H), 6.55 (d, $J=8.9 \mathrm{~Hz}, 2 \mathrm{H}), 6.77$ (d, $J=8.9$ $\mathrm{Hz}, 2 \mathrm{H}), 7.10$ (d, $J=8.3 \mathrm{~Hz}, 2 \mathrm{H}), 7.45$ (d, $J=8.3 \mathrm{~Hz}, 2 \mathrm{H}) ;{ }^{13} \mathrm{C}-\mathrm{NMR}\left(100 \mathrm{MHz}, \mathrm{CDCl}_{3}\right): \delta=$ $19.6,38.7,51.9,55.8,114.5,114.9,120.3,129.0,131.7,141.8,143.6,152.3 ; \mathrm{MS}(\mathrm{EI}): \mathrm{m} / \mathrm{z}$ $(\%)=321$ (4), 319 (4), 136 (100), 121 (4), 104 (4); HRMS: Calculated for [ $\left.\mathrm{C}_{16} \mathrm{H}_{19} \mathrm{BrNO}\right]$ : 320.064462, found 320.064714. The enantiomers were analyzed by chiral HPLC using a ODH column (n-heptane $/ 2$-Propanol $=98 / 2$, flow rate $=0.5 \mathrm{ml} / \mathrm{min}$, wavelength $=220 \mathrm{~nm}$, major enantiomer: $t_{R}=26.49$ min, minor enantiomer: $t_{R}=30.04 \mathrm{~min}$ ).

Amine 3g: purification by flash chromatography (50\% dichloro-methane in hexane). ${ }^{1} \mathrm{H}$ NMR (400 MHz, $\left.\mathrm{CDCl}_{3}\right): \delta=1.37(\mathrm{~d}, J=6.6 \mathrm{~Hz}, 3 \mathrm{H}), 3.35(\mathrm{~m}, 3 \mathrm{H}), 3.77(\mathrm{~s}, 3 \mathrm{H}), 6.61(\mathrm{~d}, J=$ 
$8.9 \mathrm{~Hz}, 2 \mathrm{H}), 6.80(\mathrm{~d}, J=8.9 \mathrm{~Hz}, 2 \mathrm{H}), 7.06(\mathrm{~m}, 1 \mathrm{H}), 7.13(\mathrm{~m}, 1 \mathrm{H}), 7.25(\mathrm{~m}, 1 \mathrm{H}) ;{ }^{13} \mathrm{C}-\mathrm{NMR}$ $\left(100 \mathrm{MHz}, \mathrm{CDCl}_{3}\right): \delta=18.4,32.8,50.8,55.8,114.4,114.9,115.6\left(\mathrm{~d}, J_{\mathrm{CF}}=23 \mathrm{~Hz}\right), 124.3(\mathrm{~d}$, $\left.J_{\mathrm{CF}}=4 \mathrm{~Hz}\right), 127.9\left(\mathrm{~d}, J_{\mathrm{CF}}=8 \mathrm{~Hz}\right), 128.2\left(\mathrm{~d}, J_{\mathrm{CF}}=5 \mathrm{~Hz}\right), 131.2\left(\mathrm{~d}, J_{\mathrm{CF}}=14 \mathrm{~Hz}\right), 142.0,152.2$, $161.1\left(\mathrm{~d}, J_{\mathrm{CF}}=246 \mathrm{~Hz}\right) ; \mathrm{MS}(\mathrm{EI}): \mathrm{m} / \mathrm{z}(\%)=259$ (11), 136 (100), 121 (5), 108 (3); HRMS: Calculated for [ $\mathrm{C}_{16} \mathrm{H}_{19} \mathrm{FNO}$ ]: 260.144517 , found 260.144509. The enantiomers were analyzed by chiral HPLC using a AS-H column (n-heptane/2-Propanol $=90 / 10$, flow rate $=0.5 \mathrm{ml} / \mathrm{min}$, wavelength $=254 \mathrm{~nm}$, major enantiomer: $t_{R}=14.67 \mathrm{~min}$, minor enantiomer: $t_{R}=18.45 \mathrm{~min}$ ).

Amine 3h: purification by flash chromatography (50\% dichloro-methane in hexane). ${ }^{1} \mathrm{H}-$ $\operatorname{NMR}\left(400 \mathrm{MHz}, \mathrm{CDCl}_{3}\right): \delta=1.33(\mathrm{~d}, J=7.2 \mathrm{~Hz}, 3 \mathrm{H}), 3.07(\mathrm{~m}, 1 \mathrm{H}), 3.20(\mathrm{dd}, J=8.1$ and $12.4 \mathrm{~Hz}, 1 \mathrm{H}), 3.30(\mathrm{dd}, J=6.1$ and $12.4 \mathrm{~Hz}, 1 \mathrm{H}), 3.75(\mathrm{~s}, 3 \mathrm{H}), 6.56(\mathrm{~d}, J=8.9 \mathrm{~Hz}, 2 \mathrm{H}), 6.78$ $(\mathrm{d}, J=8.9 \mathrm{~Hz}, 2 \mathrm{H}), 6.95(\mathrm{~m}, 2 \mathrm{H}), 7.01$ (d, $J=7.8 \mathrm{~Hz}, 1 \mathrm{H}), 7.30(\mathrm{~m}, 1 \mathrm{H}) ;{ }^{13} \mathrm{C}-\mathrm{NMR}(100$ $\left.\mathrm{MHz}, \mathrm{CDCl}_{3}\right): \delta=19.4,39.0,51.9,55.7,113.4\left(\mathrm{~d}, J_{\mathrm{CF}}=21 \mathrm{~Hz}\right), 113.9\left(\mathrm{~d}, J_{\mathrm{CF}}=21 \mathrm{~Hz}\right), 114.4$, $114.9,122.9\left(\mathrm{~d}, J_{\mathrm{CF}}=3 \mathrm{~Hz}\right), 130.0\left(\mathrm{~d}, J_{\mathrm{CF}}=8 \mathrm{~Hz}\right), 141.9,147.3\left(\mathrm{~d}, J_{\mathrm{CF}}=7 \mathrm{~Hz}\right), 152.2,163.0$ $\left(\mathrm{d}, J_{\mathrm{CF}}=244 \mathrm{~Hz}\right) ; \mathrm{MS}(\mathrm{EI}): \mathrm{m} / \mathrm{z}(\%)=259$ (11), 136 (100), 121 (5), 108 (3); HRMS: Calculated for $\left[\mathrm{C}_{16} \mathrm{H}_{18} \mathrm{FNNaO}\right]$ : 282.126459, found 282.126642. The enantiomers were analyzed by chiral HPLC using a OD-H column (n-heptane/2-Propanol $=98 / 2$, flow rate $=0.5$ $\mathrm{ml} / \mathrm{min}$, wavelength $=220 \mathrm{~nm}$, major enantiomer: $t_{R}=22.90 \min$, minor enantiomer: $t_{R}=$ $26.63 \mathrm{~min})$.

Amine 3i: purification by flash chromatography (50\% dichloromethane in hexane). ${ }^{1} \mathrm{H}-\mathrm{NMR}$ (400 MHz, $\left.\mathrm{CDCl}_{3}\right): \delta=1.42(\mathrm{~d}, J=6.5 \mathrm{~Hz}, 3 \mathrm{H}), 3.21(\mathrm{dd}, J=7.5,12.0 \mathrm{~Hz}, 1 \mathrm{H}), 3.35(\mathrm{~m}, 2 \mathrm{H})$, $3.76(\mathrm{~s}, 3 \mathrm{H}), 6.59$ (d, $J=8.9 \mathrm{~Hz}, 2 \mathrm{H}), 6.79$ (d, $J=8.9 \mathrm{~Hz}, 2 \mathrm{H}), 6.87(\mathrm{~d}, J=3.6 \mathrm{~Hz}, 1 \mathrm{H}), 6.97$ $(\mathrm{dd}, J=3.6$ and $5.1 \mathrm{~Hz}, 1 \mathrm{H}), 7.20(\mathrm{~d}, J=5.1 \mathrm{~Hz}, 1 \mathrm{H}) ;{ }^{13} \mathrm{C}-\mathrm{NMR}\left(100 \mathrm{MHz}, \mathrm{CDCl}_{3}\right): \delta=20.6$, $34.9,52.8,55.8,114.5,114.9,123.3,123.6,126.7,142.0,148.5,152.2 ; \mathrm{MS}(\mathrm{EI}): \mathrm{m} / \mathrm{z}(\%)=$ 247 (10), 136 (100), 121 (5), 108 (3); HRMS: Calculated for [ $\mathrm{C}_{14} \mathrm{H}_{17} \mathrm{NNaOS}$ ]: 270.092302, found 270.091905. The enantiomers were analyzed by chiral HPLC using a AD-H column (nheptane/2-Propanol $=99.5 / 0.5$, flow rate $=0.5 \mathrm{ml} / \mathrm{min}$, wavelength $=220 \mathrm{~nm}$, major enantiomer: $t_{R}=33.26$ min, minor enantiomer: $t_{R}=29.79 \mathrm{~min}$ ).

Amine 3j: purification by flash chromatography (3\% EtOAc in hexane). ${ }^{1} \mathrm{H}-\mathrm{NMR}(400 \mathrm{MHz}$, $\left.\mathrm{CDCl}_{3}\right): \delta=0.93(\mathrm{~d}, J=6.8 \mathrm{~Hz}, 3 \mathrm{H}), 1.20(\mathrm{~m}, 6 \mathrm{H}), 1.68(\mathrm{~m}, 6 \mathrm{H}), 2.85(\mathrm{dd}, J=7.8$ and 11.9 $\mathrm{Hz}, 1 \mathrm{H}), 3.11(\mathrm{dd}, J=5.4$ and $11.9 \mathrm{~Hz}, 1 \mathrm{H}), 3.75(\mathrm{~s}, 3 \mathrm{H}), 6.58(\mathrm{~d}, J=9.0 \mathrm{~Hz}, 2 \mathrm{H}), 6.78$ (d, $J$ $=9.0 \mathrm{~Hz}, 2 \mathrm{H}) ;{ }^{13} \mathrm{C}-\mathrm{NMR}\left(100 \mathrm{MHz}, \mathrm{CDCl}_{3}\right): \delta=14.8,26.7,26.7,26.8,28.7,30.9,38.0,40.8$, 
49.0, 55.9, 114.0, 114.9, 142.9, 151.9; MS (EI):m/z (\%) = 247 (22), 136 (100), 121 (3), 108 (3); HRMS: Calculated for $\left[\mathrm{C}_{16} \mathrm{H}_{25} \mathrm{NNaO}\right]: 270.182836$, found 270.182714. The enantiomers were analyzed by chiral HPLC using a AD-H column (n-heptane/2-Propanol = 99/1, flow rate $=0.5 \mathrm{ml} / \mathrm{min}$, wavelength $=220 \mathrm{~nm}$, major enantiomer: $t_{\mathrm{R}}=25.61 \mathrm{~min}$, minor enantiomer: $\mathrm{t}_{\mathrm{R}}$ $=19.43 \mathrm{~min})$.

Amine 3k: purification by flash chromatography (3\% EtOAc in hexane). ${ }^{1} \mathrm{H}-\mathrm{NMR}$ (400 $\left.\mathrm{MHz}, \mathrm{CDCl}_{3}\right): \delta=0.93(\mathrm{~s}, 9 \mathrm{H}), 0.97(\mathrm{~d}, J=6.6 \mathrm{~Hz}, 3 \mathrm{H}), 1.47(\mathrm{~m}, 1 \mathrm{H}), 2.67(\mathrm{dd}, J=10.0$ and $11.8 \mathrm{~Hz}, 1 \mathrm{H}), 3.31(\mathrm{dd}, J=2.9$ and $11.8 \mathrm{~Hz}, 1 \mathrm{H}), 3.75(\mathrm{~s}, 3 \mathrm{H}), 6.58(\mathrm{~d}, J=8.9 \mathrm{~Hz}, 2 \mathrm{H}), 6.79$ $(\mathrm{d}, J=8.9 \mathrm{~Hz}, 2 \mathrm{H}) ;{ }^{13} \mathrm{C}-\mathrm{NMR}\left(100 \mathrm{MHz}, \mathrm{CDCl}_{3}\right): \delta=13.5,27.5,32.5,42.7,47.3,55.9,114.0$, 114.9, 143.0, 151.8; MS (EI):m/z (\%) = 221 (20), 136 (100), 121 (3), 108 (3); HRMS: Calculated for [ $\mathrm{C}_{14} \mathrm{H}_{23} \mathrm{NO}$ ]: 221.177961, found 221.177832. The enantiomers were analyzed by chiral HPLC using a OD-H column (n-heptane/2-Propanol $=99 / 1$, flow rate $=0.5 \mathrm{ml} / \mathrm{min}$, wavelength $=220 \mathrm{~nm}$, major enantiomer: $t_{R}=14.55 \mathrm{~min}$, minor enantiomer: $t_{R}=13.04 \mathrm{~min}$ ).

Amine 31: purification by flash chromatography (4\% EtOAc in hexane). ${ }^{1} \mathrm{H}-\mathrm{NMR}(300 \mathrm{MHz}$, $\left.\mathrm{CDCl}_{3}\right): \delta=1.10(\mathrm{~d}, J=7.0 \mathrm{~Hz}, 3 \mathrm{H}), 2.33-2.53(\mathrm{~m}, 1 \mathrm{H}), 3.02(\mathrm{dd}, J=7.3$ and $13.6 \mathrm{~Hz}, 1 \mathrm{H})$, $3.37(\mathrm{dd}, J=5.5$ and $13.6 \mathrm{~Hz}, 1 \mathrm{H}), 3.68(\mathrm{~s}, 3 \mathrm{H}), 6.50(\mathrm{~d}, J=6.6 \mathrm{~Hz}, 2 \mathrm{H}), 6.71(\mathrm{~d}, J=6.6 \mathrm{~Hz}$, $2 \mathrm{H}) ;{ }^{13} \mathrm{C}-\mathrm{NMR}\left(75 \mathrm{MHz} \mathrm{CDCl}_{3}\right): \delta=11.6,37.6(\mathrm{q}, J=25.1 \mathrm{~Hz}), 44.8,55.9,114.5,115.2$, 128.0 (q, $J=277.8 \mathrm{~Hz}), 141.4,152.7$; MS (EI):m/z (\%) = 233 (35), 218 (6), 136 (100), 121 (8), 108 (5); HRMS: Calculated for $\left[\mathrm{C}_{11} \mathrm{H}_{14} \mathrm{~F}_{3} \mathrm{NO}\right]$ : 233.102748, found 233.102778. The enantiomers were analyzed by chiral HPLC using a AS-H column (n-heptane/2-Propanol = $98 / 2$, flow rate $=0.5 \mathrm{ml} / \mathrm{min}$, wavelength $=220 \mathrm{~nm}$, major enantiomer: $t_{R}=26.09 \mathrm{~min}$, minor enantiomer: $\left.\mathrm{t}_{\mathrm{R}}=22.91 \mathrm{~min}\right)$.

Amine 3m: purification by flash chromatography (3\% EtOAc in hexane). ${ }^{1} \mathrm{H}-\mathrm{NMR}$ (400 $\left.\mathrm{MHz}, \mathrm{CDCl}_{3}\right): \delta=0.93(\mathrm{t}, J=7.2 \mathrm{~Hz}, 3 \mathrm{H}), 0.97(\mathrm{~d}, J=6.7 \mathrm{~Hz}, 3 \mathrm{H}), 1.30(\mathrm{~m}, 4 \mathrm{H}), 1.76(\mathrm{~m}$, 1H), $2.85(\mathrm{dd}, J=7.2$ and $12.0 \mathrm{~Hz}, 1 \mathrm{H}), 3.03(\mathrm{dd}, J=5.8$ and $12.0 \mathrm{~Hz}, 1 \mathrm{H}), 3.76(\mathrm{~s}, 3 \mathrm{H}), 6.60$ $(\mathrm{d}, J=8.9 \mathrm{~Hz}, 2 \mathrm{H}), 6.80(\mathrm{~d}, J=8.9 \mathrm{~Hz}, 2 \mathrm{H}) ;{ }^{13} \mathrm{C}-\mathrm{NMR}\left(100 \mathrm{MHz}, \mathrm{CDCl}_{3}\right): \delta=14.3,18.0$, 20.1, 32.7, 37.1, 51.3, 55.8, 113.8, 114.9, 142.9, 151.8; MS (EI):m/z (\%) = 207 (19), 136 (100), 121 (3), 108 (3); HRMS: Calculated for [ $\mathrm{C}_{13} \mathrm{H}_{21} \mathrm{NO}$ ]: 207.162312, found 207.162495. The enantiomers were analyzed by chiral HPLC using a OJ-H column (n-heptane/2-Propanol $=99.5 / 0.5$, flow rate $=0.5 \mathrm{ml} / \mathrm{min}$, wavelength $=220 \mathrm{~nm}$, major enantiomer: $\mathrm{t}_{\mathrm{R}}=58.04 \mathrm{~min}$, minor enantiomer: $\left.t_{R}=51.71 \mathrm{~min}\right)$. 
Amine 3n: purification by flash chromatography (5\% EtOAc in hexane). ${ }^{1} \mathrm{H}-\mathrm{NMR}$ (400 $\left.\mathrm{MHz}, \mathrm{CDCl}_{3}\right): \delta=0.87(\mathrm{t}, J=7.4 \mathrm{~Hz}, 3 \mathrm{H}), 1.69(\mathrm{~m}, 1 \mathrm{H}), 1.82(\mathrm{~m}, 1 \mathrm{H}), 2.82(\mathrm{~m}, 1 \mathrm{H}), 3.20(\mathrm{dd}$, $J=9.1$ and $12.1 \mathrm{~Hz}, 1 \mathrm{H}), 3.43(\mathrm{dd}, J=5.3$ and $12.1 \mathrm{~Hz}, 1 \mathrm{H}), 3.77(\mathrm{~s}, 3 \mathrm{H}), 6.56(\mathrm{~d}, J=8.9 \mathrm{~Hz}$, 2H), $6.79(\mathrm{~d}, J=8.9 \mathrm{~Hz}, 2 \mathrm{H}), 7.30(\mathrm{~m}, 3 \mathrm{H}) ;{ }^{13} \mathrm{C}-\mathrm{NMR}\left(100 \mathrm{MHz}, \mathrm{CDCl}_{3}\right): \delta=12.0,27.2,47.2$, $50.5,55.8,114.4,114.9,126.6,127.9,128.6,142.4,143.0,152.1 ; \mathrm{MS}(\mathrm{EI}): \mathrm{m} / \mathrm{z}(\%)=255$ (13), 136 (100), 121 (4), 108 (3), 91 (5); HRMS: Calculated for $\left[\mathrm{C}_{17} \mathrm{H}_{21} \mathrm{NO}\right]$ : 255.162315, found 255.162552. The enantiomers were analyzed by chiral HPLC using a OD-H column (nheptane $/ 2$-Propanol $=98 / 2$, flow rate $=0.5 \mathrm{ml} / \mathrm{min}$, wavelength $=220 \mathrm{~nm}$, major enantiomer: $t_{R}=18.58$ min, minor enantiomer: $t_{R}=20.14$ min).

Amine 3o: purification by flash chromatography (3\% EtOAc in hexane). ${ }^{1} \mathrm{H}-\mathrm{NMR}(400 \mathrm{MHz}$, $\left.\mathrm{CDCl}_{3}\right): \delta=1.38(\mathrm{~d}, J=6.8 \mathrm{~Hz}, 3 \mathrm{H}), 3.10(\mathrm{~m}, 1 \mathrm{H}), 3.29(\mathrm{dd}, J=8.1$ and $12.4 \mathrm{~Hz}, 1 \mathrm{H}), 3.39$ (dd, $J=6.3$ and $12.4 \mathrm{~Hz}, 1 \mathrm{H}), 3.77(\mathrm{~s}, 1 \mathrm{H}), 6.62(\mathrm{~d}, J=8.4 \mathrm{~Hz}, 2 \mathrm{H}), 6.74(\mathrm{t}, J=7.3 \mathrm{~Hz}, 1 \mathrm{H})$, $7.19(\mathrm{~m}, 2 \mathrm{H}), 7.24(\mathrm{~m}, 3 \mathrm{H}), 7.37(\mathrm{~m}, 2 \mathrm{H}) ;{ }^{13} \mathrm{C}-\mathrm{NMR}\left(100 \mathrm{MHz}, \mathrm{CDCl}_{3}\right): \delta=19.7,39.2,51.0$, 113.0, 117.5, 126.6, 127.2, 128.7, 129.2, 144.5, 147.9; MS (EI):m/z (\%) = $211(11), 106$ (100), 91 (2), 77 (11); HRMS: Calculated for $\left[\mathrm{C}_{15} \mathrm{H}_{17} \mathrm{~N}\right]$ : 211.136096, found 211.136286. The enantiomers were analyzed by chiral HPLC using a AD-H column (n-heptane/2-Propanol = $95 / 5$, flow rate $=0.5 \mathrm{ml} / \mathrm{min}$, wavelength $=220 \mathrm{~nm}$, major enantiomer: $t_{\mathrm{R}}=10.63 \mathrm{~min}$, minor enantiomer: $\left.t_{R}=9.84 \mathrm{~min}\right)$.

Amine 3p: purification by flash chromatography (3\% EtOAc in hexane). ${ }^{1} \mathrm{H}-\mathrm{NMR}$ (400 $\left.\mathrm{MHz}, \mathrm{CDCl}_{3}\right): \delta=1.38(\mathrm{~d}, J=7.0 \mathrm{~Hz}, 3 \mathrm{H}), 3.10(\mathrm{~m}, 1 \mathrm{H}), 3.30(\mathrm{dd}, J=8.3$ and $12.4 \mathrm{~Hz}, 1 \mathrm{H})$, $3.41(\mathrm{dd}, J=6.2$ and $12.4 \mathrm{~Hz}, 1 \mathrm{H}), 3.94(\mathrm{~s}, 1 \mathrm{H}), 6.60(\mathrm{~d}, J=8.6 \mathrm{~Hz}, 2 \mathrm{H}), 7.27(\mathrm{~m}, 3 \mathrm{H}), 7.38$ $(\mathrm{m}, 4 \mathrm{H}) ;{ }^{13} \mathrm{C}-\mathrm{NMR}\left(100 \mathrm{MHz}, \mathrm{CDCl}_{3}\right): \delta=19.6,39.1,50.3,111.9,118.5\left(\mathrm{q}, J_{\mathrm{CF}}=33 \mathrm{~Hz}\right)$, $124.9\left(\mathrm{q}, J_{\mathrm{CF}}=270 \mathrm{~Hz}\right), 126.5\left(\mathrm{q}, J_{\mathrm{CF}}=4 \mathrm{~Hz}\right), 126.8,127.2,128.8,144.0,150.5 ; \mathrm{MS}(\mathrm{EI}): \mathrm{m} / \mathrm{z}$ $(\%)=279$ (12), 174 (100), 145 (5), 127 (2); HRMS: Calculated for $\left[\mathrm{C}_{16} \mathrm{H}_{16} \mathrm{~F}_{3} \mathrm{~N}\right]: 279.123484$, found 279.123744. The enantiomers were analyzed by chiral HPLC using a AD-H column (nheptane $/ 2$-Propanol $=95 / 5$, flow rate $=0.5 \mathrm{ml} / \mathrm{min}$, wavelength $=220 \mathrm{~nm}$, major enantiomer: $t_{R}=13.04$ min, minor enantiomer: $\left.t_{R}=11.71 \mathrm{~min}\right)$.

Preparation of dihydropyridine 4c: A mixture of ammonium acetate (10.1, $129 \mathrm{mmol})$, hexa-methylenetetramine $(18.1 \mathrm{~g}, 129 \mathrm{mmol})$, methylacetoacetate $(15,129 \mathrm{mmol})$ and tert.butylacetoacetate $(20.44 \mathrm{~g}, 129 \mathrm{mmol})$ was stirred for 30 minutes at $100{ }^{\circ} \mathrm{C}$. Volatile 
compounds were removed under reduced pressure, the reaction mixture was diluted with dichloromethane and extracted with water $(3 \times 100 \mathrm{ml})$. Then, the solvent was removed and the product was purified by flash chromatography (5\% EtOAc in hexane), to afford pure 4 $(7.1 \mathrm{~g}, 21 \%)$.

${ }^{1} \mathrm{H}-\mathrm{NMR}\left(400 \mathrm{MHz}, \mathrm{CDCl}_{3}\right): \delta=1.47(\mathrm{~s}, 9 \mathrm{H}), 2.14(\mathrm{~s}, 3 \mathrm{H}), 2.18(\mathrm{~s}, 3 \mathrm{H}), 3.20(\mathrm{~s}, 2 \mathrm{H}), 3.68(\mathrm{~s}$, $3 \mathrm{H}), 5.17$ (brs, 1H).

\section{Cleavage of the PMP-protecting group and determination of the absolute configuration} of 3a:

\section{a.) CbZ-protection/PMP-deprotection sequence ${ }^{15}$ :}

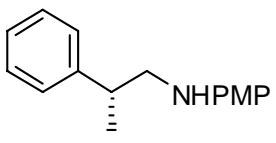

(R)-3a, 98:2 er

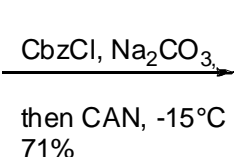

Benzyl chloroformate $(0.157 \mathrm{ml}, 1.12 \mathrm{mmol})$ and $\mathrm{Na}_{2} \mathrm{CO}_{3}(1.0 \mathrm{ml}, 4 \mathrm{M})$ were added to a mixture of $3 \mathrm{a}(0.241 \mathrm{~g}, 1.0 \mathrm{mmol})$, THF $(1 \mathrm{ml})$ and $\mathrm{Na}_{2} \mathrm{CO}_{3}(2.5 \mathrm{ml}, 4 \mathrm{M})$ at $0^{\circ} \mathrm{C}$. After stirring at room temperature for 3 hours, water $(5 \mathrm{ml})$ was added and the mixture extracted with ethyl acetate $(3 \times 5 \mathrm{ml})$. The combined extracts were dried $\left(\mathrm{Na}_{2} \mathrm{SO}_{4}\right)$ and concentrated in vacuo. The residue was dissolved in $\mathrm{CH}_{3} \mathrm{CN}(7 \mathrm{ml})$ and cooled to $-15{ }^{\circ} \mathrm{C}$. A solution of CAN $(1.64 \mathrm{~g}, 3.0 \mathrm{mmol})$ in water $(12 \mathrm{ml})$ was added dropwise $(20 \mathrm{~min})$ and the mixture stirred at $10{ }^{\circ} \mathrm{C}$ for $30 \mathrm{~min}$. Water $(20 \mathrm{ml})$ was added, and the mixture extracted with ethyl acetate $(3 \mathrm{x}$ $15 \mathrm{ml}$ ). The combined organic extracts were washed with a saturated $\mathrm{NaHCO}_{3}$-solution (30 $\mathrm{ml})$ and the aqueous solution was extracted with ethyl acetate $(2 \mathrm{x} 15 \mathrm{ml})$. The organic phases were washed with saturated $\mathrm{Na}_{2} \mathrm{SO}_{3}$-solution $(30 \mathrm{ml})$, saturated $\mathrm{NaHCO}_{3}$-solution $(30 \mathrm{ml})$, brine $(30 \mathrm{ml})$, dried $\left(\mathrm{Na}_{2} \mathrm{SO}_{4}\right)$ and concentrated in vacuo. The residue was purified by flash chromatography. (5\% EtOAc in hexane) to yield a clear oil $(0.190 \mathrm{~g}, 71 \%)$.

${ }^{1} \mathrm{H}-\mathrm{NMR}\left(300 \mathrm{MHz}, \mathrm{CDCl}_{3}\right): \delta=1.26(\mathrm{~d}, J=7.0 \mathrm{~Hz}, 3 \mathrm{H}), 2.93(\mathrm{~m}, 1 \mathrm{H}), 3.25(\mathrm{~m}, 1 \mathrm{H}), 3.48$ $(\mathrm{m}, 1 \mathrm{H}), 4.67(\mathrm{~s} b r, 1 \mathrm{H}), 5.06(\mathrm{~s}, 2 \mathrm{H}), 7.16-7.36(\mathrm{~m}, 10 \mathrm{H}) ;{ }^{13} \mathrm{C}-\mathrm{NMR}\left(100 \mathrm{MHz}, \mathrm{CDCl}_{3}\right): \delta=$ $19.6,40.0,47.7,66.6,126.7,127.2,128.1,128.5,128.7,136.5,143.9,156.3 ; \mathrm{MS}(\mathrm{EI}): \mathrm{m} / \mathrm{z}$ $(\%)=269$ (4), 208 (2), 195 (7), 178 (28), 105 (37), 91 (100); HRMS: Calculated for $\left[\mathrm{C}_{17} \mathrm{H}_{19} \mathrm{NO}_{2}\right]$ : 269.141581 , found 269.141375 . The enantiomers were analyzed by chiral

\footnotetext{
${ }^{15}$ Niwa, Y.; Takayama, K.; Shimizu, M. Bull. Chem. Soc. Jpn. 2002, 75, 1819-1825.
} 
HPLC using a OJ-H column (n-heptane/2-Propanol $=85 / 15$, flow rate $=0.5 \mathrm{ml} / \mathrm{min}$, wavelength $=210 \mathrm{~nm}$, major enantiomer: $t_{R}=32.93 \mathrm{~min}$, minor enantiomer: $t_{R}=29.55 \mathrm{~min}$ ).

\section{b.) PMP-deprotection/BOC-protection sequence:}

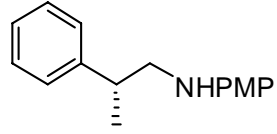

$(R)-3 \mathbf{a}, 98: 2$ er

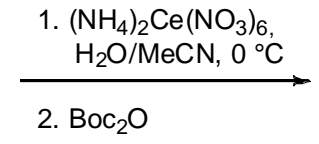

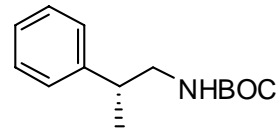

$(R)-6 \mathbf{b}, 98: 2$ er

Amine 3a (241 mg, $1.0 \mathrm{mmol})$ was dissolved in acetonitrile $(25 \mathrm{ml})$ and added to an aqueous cerium ammonium nitrate solution $\left(2.74 \mathrm{~g}, 5.0 \mathrm{mmol}, 20 \mathrm{ml} \mathrm{H}_{2} \mathrm{O}\right)$ at $0{ }^{\circ} \mathrm{C}$, the reaction mixture was then stirred for 4 hours at room temperature. $\mathrm{Boc}_{2} \mathrm{O}(2.0 \mathrm{~g}, 12.5 \mathrm{mmol})$ was added portion wise and the solution stirred overnight. The mixture was quenched with water, neutralized with a $\mathrm{NaHCO}_{3}$-solution (saturated) and extracted with ethylacetate. After removing the solvent, the crude product was purified by column chromatography to yield the title compound as a clear oil (123 mg, 52\%).

By comparison of the HPLC-retention time with a known sample, the configuration was determined. The known sample of $\mathbf{6}$ was prepared by introducing a Boc-protecting group to (R)- $\beta$-Methylphenethylamine.

${ }^{1} \mathrm{H}-\mathrm{NMR}\left(400 \mathrm{MHz}, \mathrm{CDCl}_{3}\right): \delta=1.26(\mathrm{~d}, J=7.0 \mathrm{~Hz}, 3 \mathrm{H}), 1.41(\mathrm{~s}, 9 \mathrm{H}), 2.91(\mathrm{~m}, 1 \mathrm{H}), 3.18(\mathrm{~m}$, 1H), $3.40(\mathrm{~m}, 1 \mathrm{H}), 4.44$ (brs, $1 \mathrm{H}), 7.26(\mathrm{~m}, 5 \mathrm{H}) ;{ }^{13} \mathrm{C}-\mathrm{NMR}\left(100 \mathrm{MHz}, \mathrm{CDCl}_{3}\right): \delta=19.1,28.3$, 40.1, 47.3, 78.8, 126.5, 127.2, 128.6, 144.2, 155.9; MS (EI):m/z (\%) = 235 (2), 179 (37), 130 (6), 118 (13), 105 (30), 91 (12), 57 (100); HRMS: Calculated for $\left[\mathrm{C}_{14} \mathrm{H}_{21} \mathrm{NO}_{2}\right]$ : 235.157232, found 235.156972. The enantiomers were analyzed by chiral HPLC using a AD-H column (nheptane $/ 2$-Propanol $=99 / 1$, flow rate $=0.5 \mathrm{ml} / \mathrm{min}$, wavelength $=220 \mathrm{~nm}$, major enantiomer: $t_{R}=17.16$ min, minor enantiomer: $\left.t_{R}=18.64 \mathrm{~min}\right)$. 

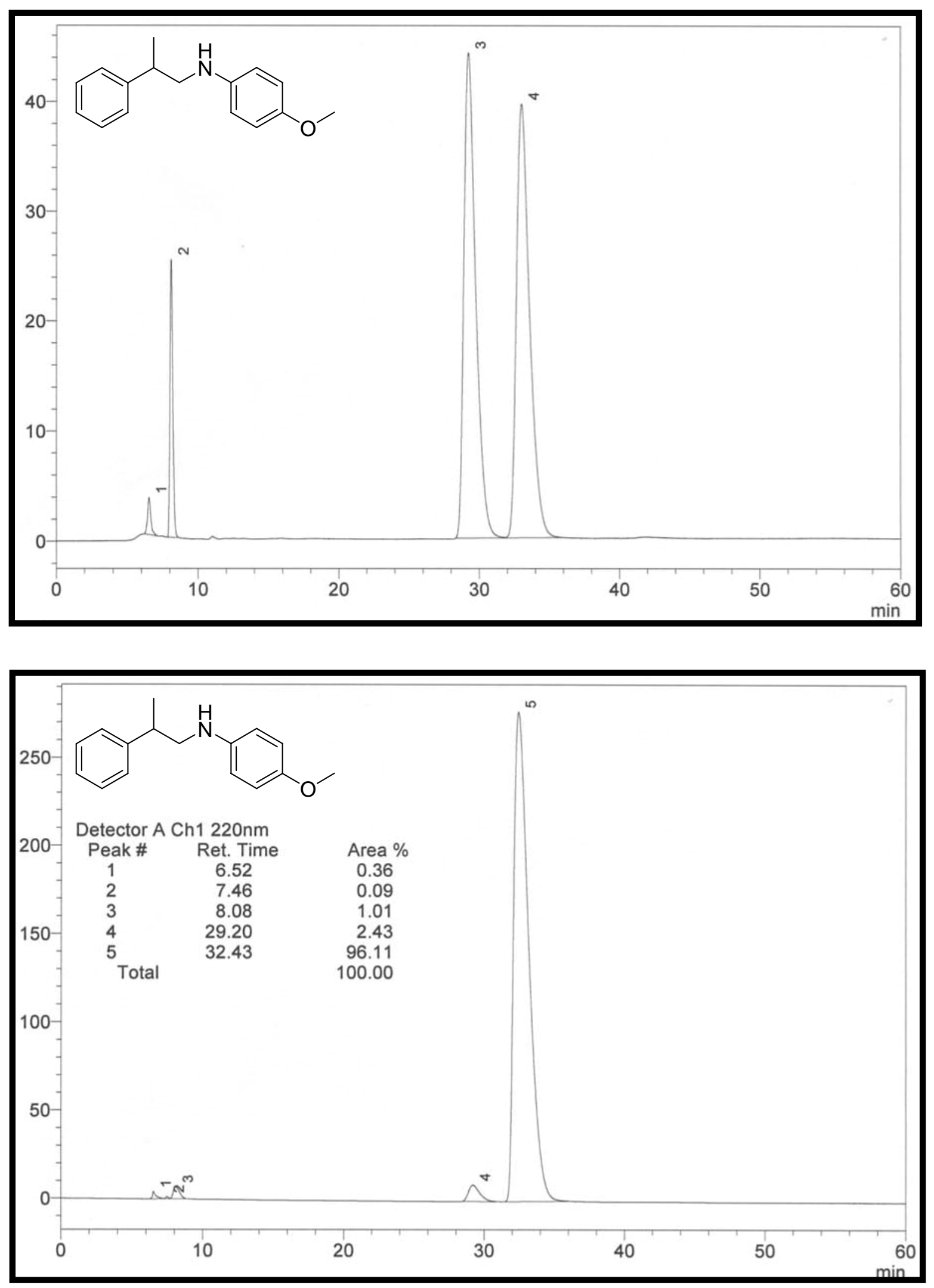


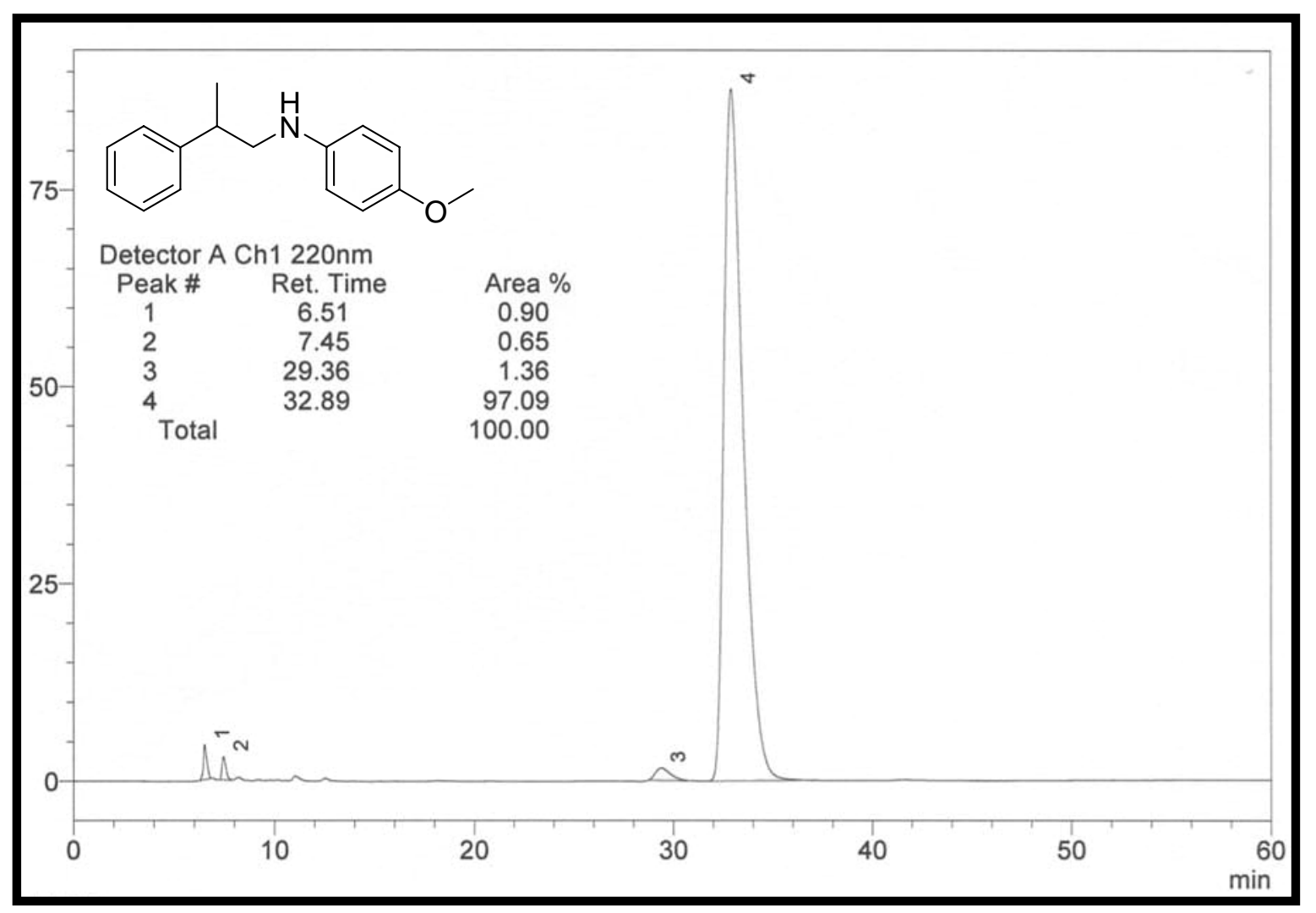



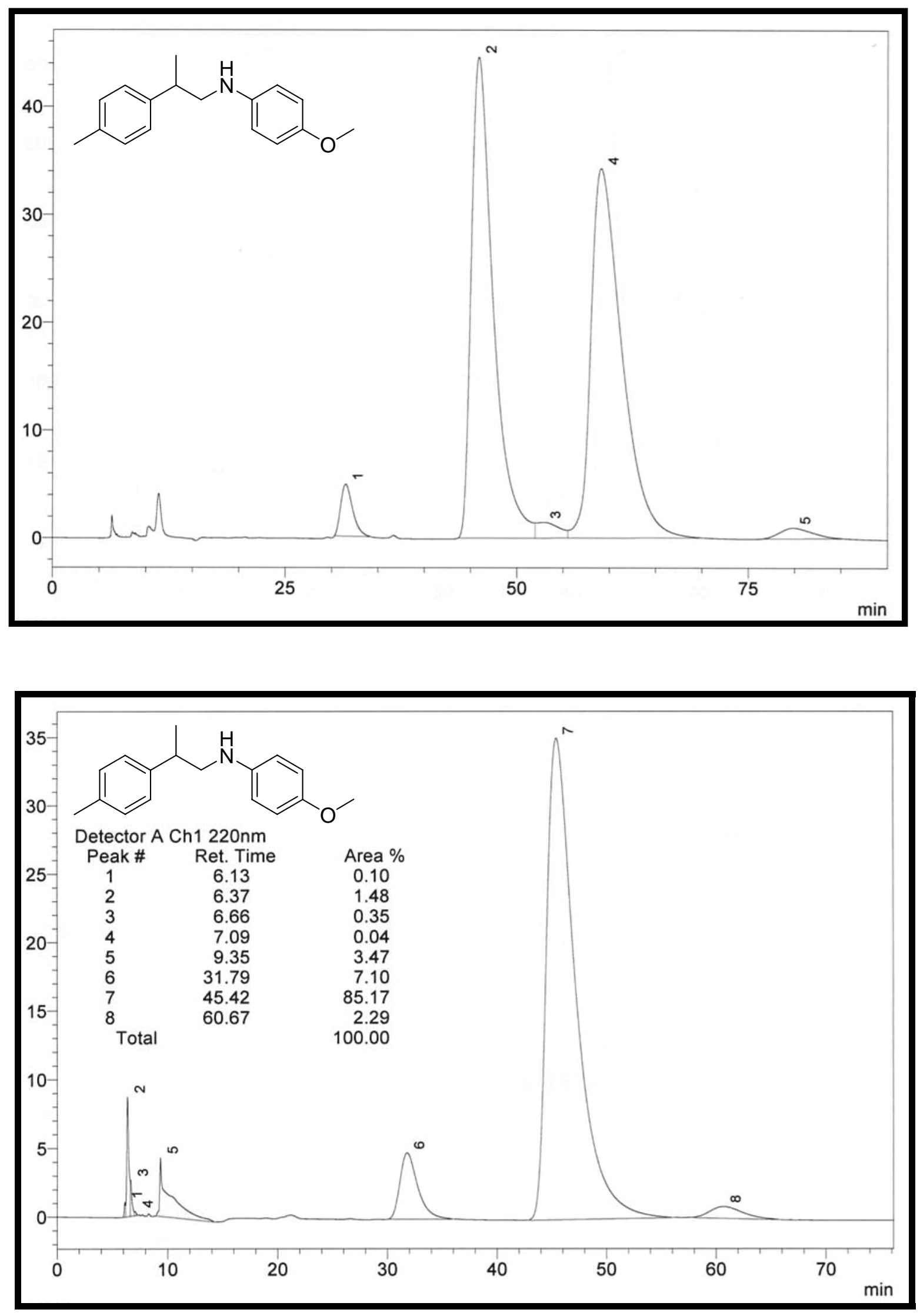

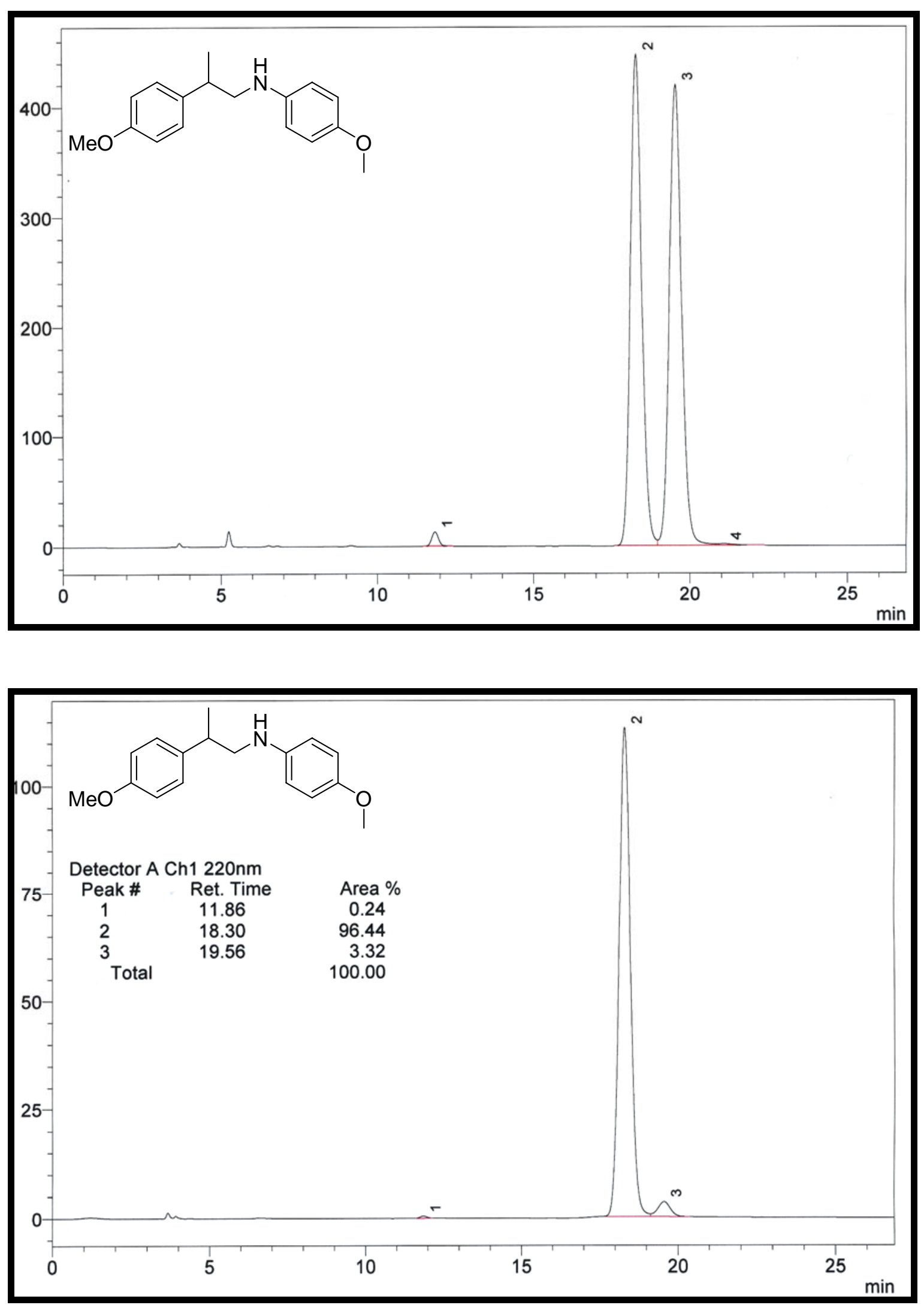

- S18 - 

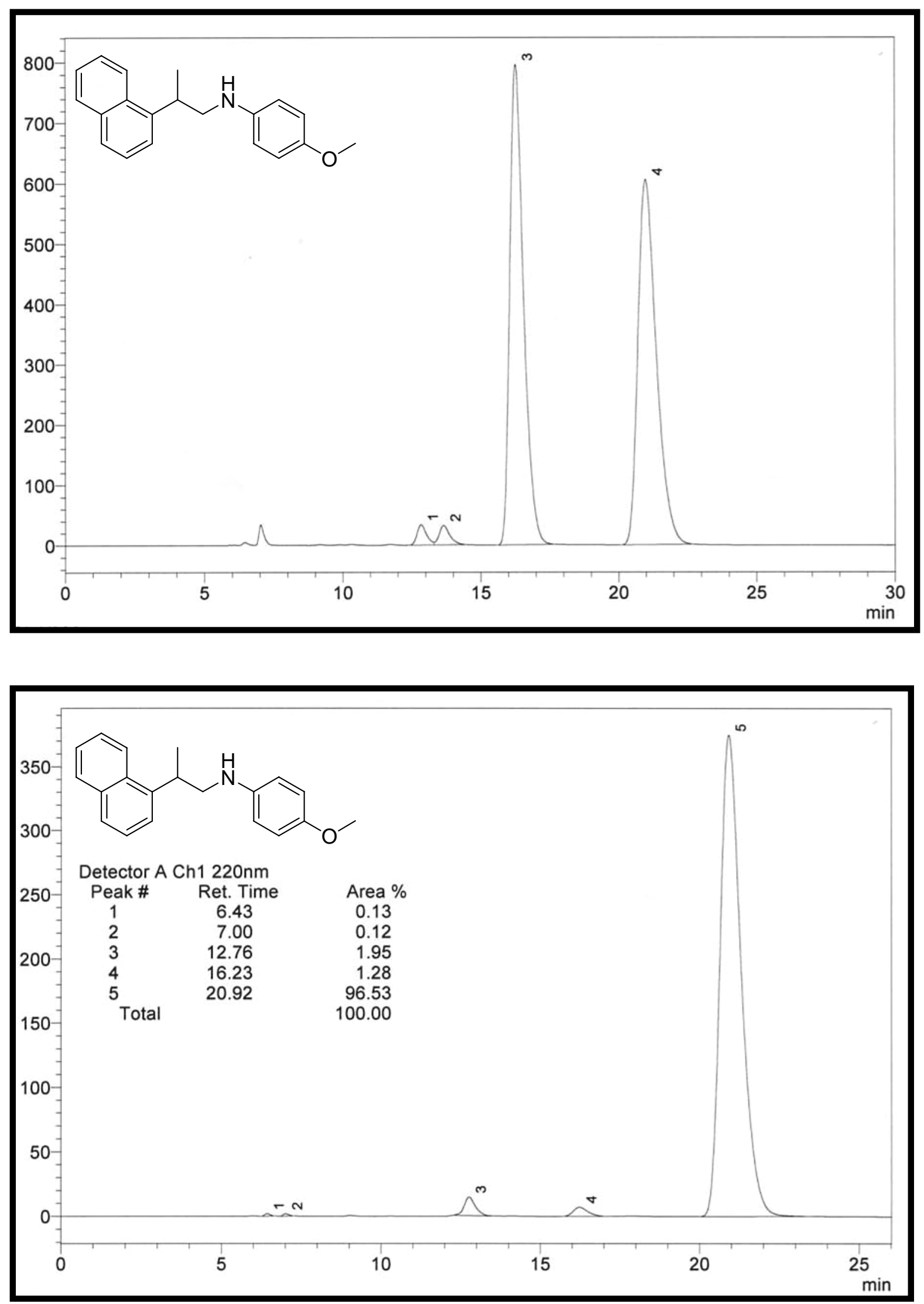

- S19 - 

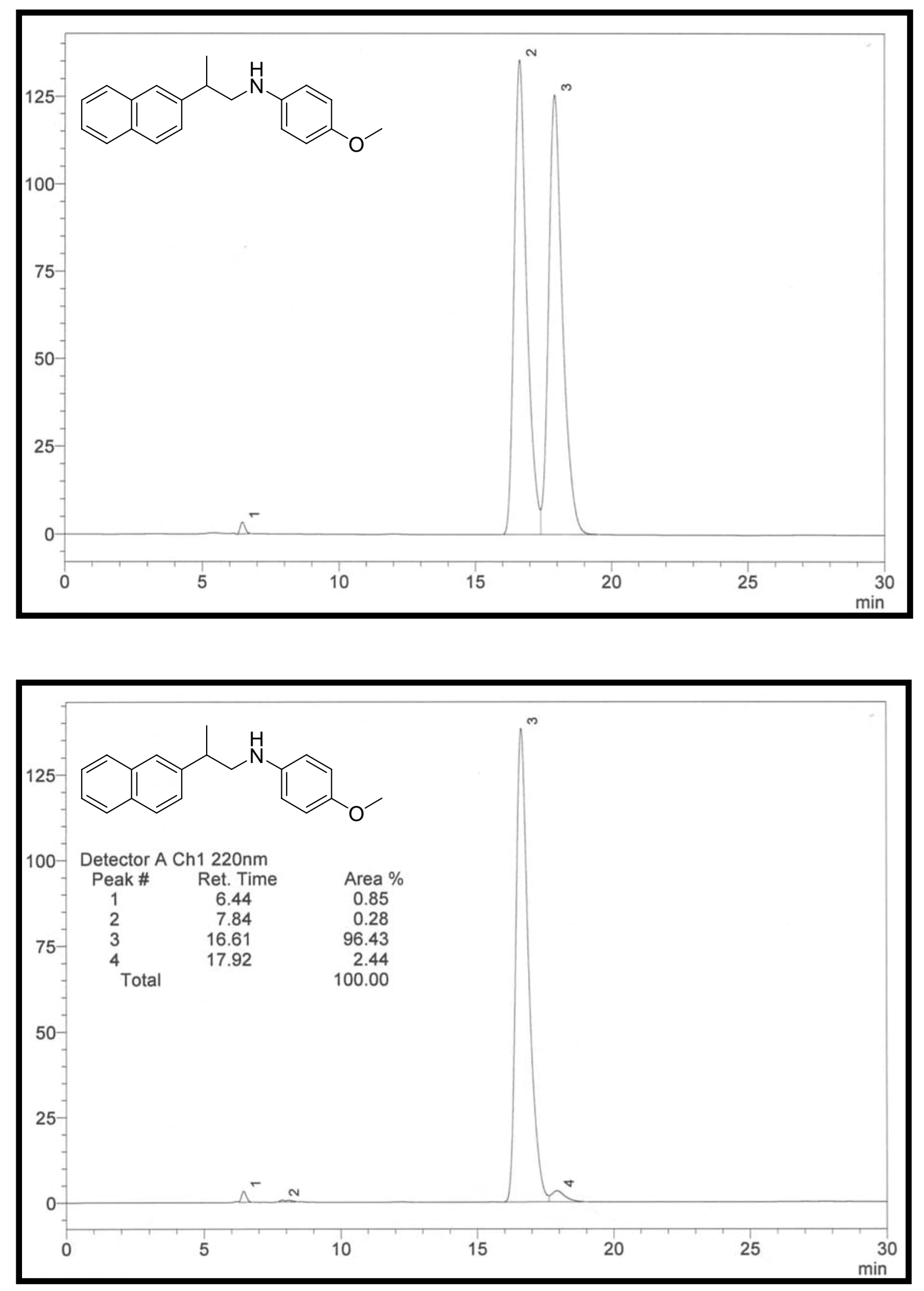

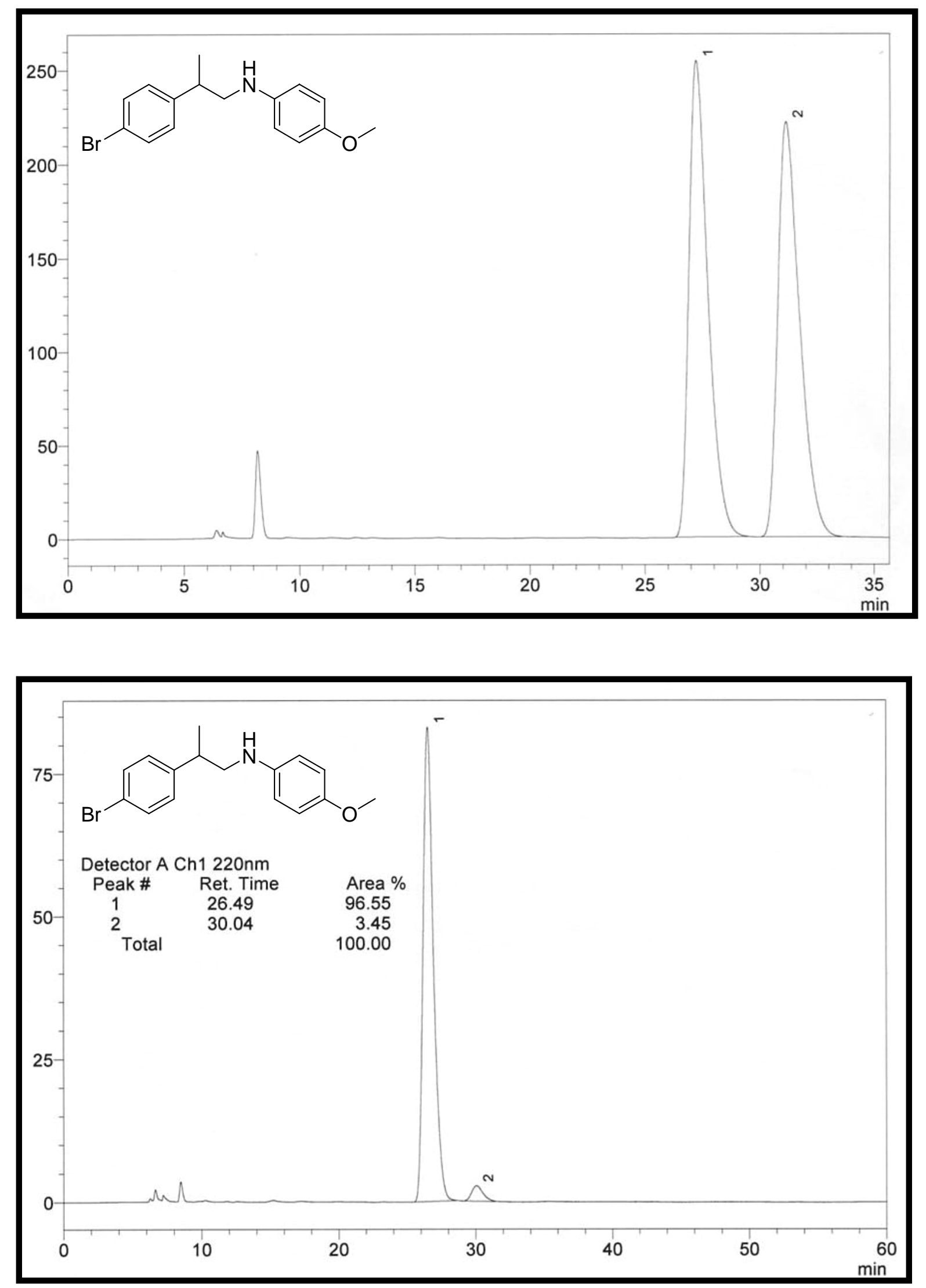

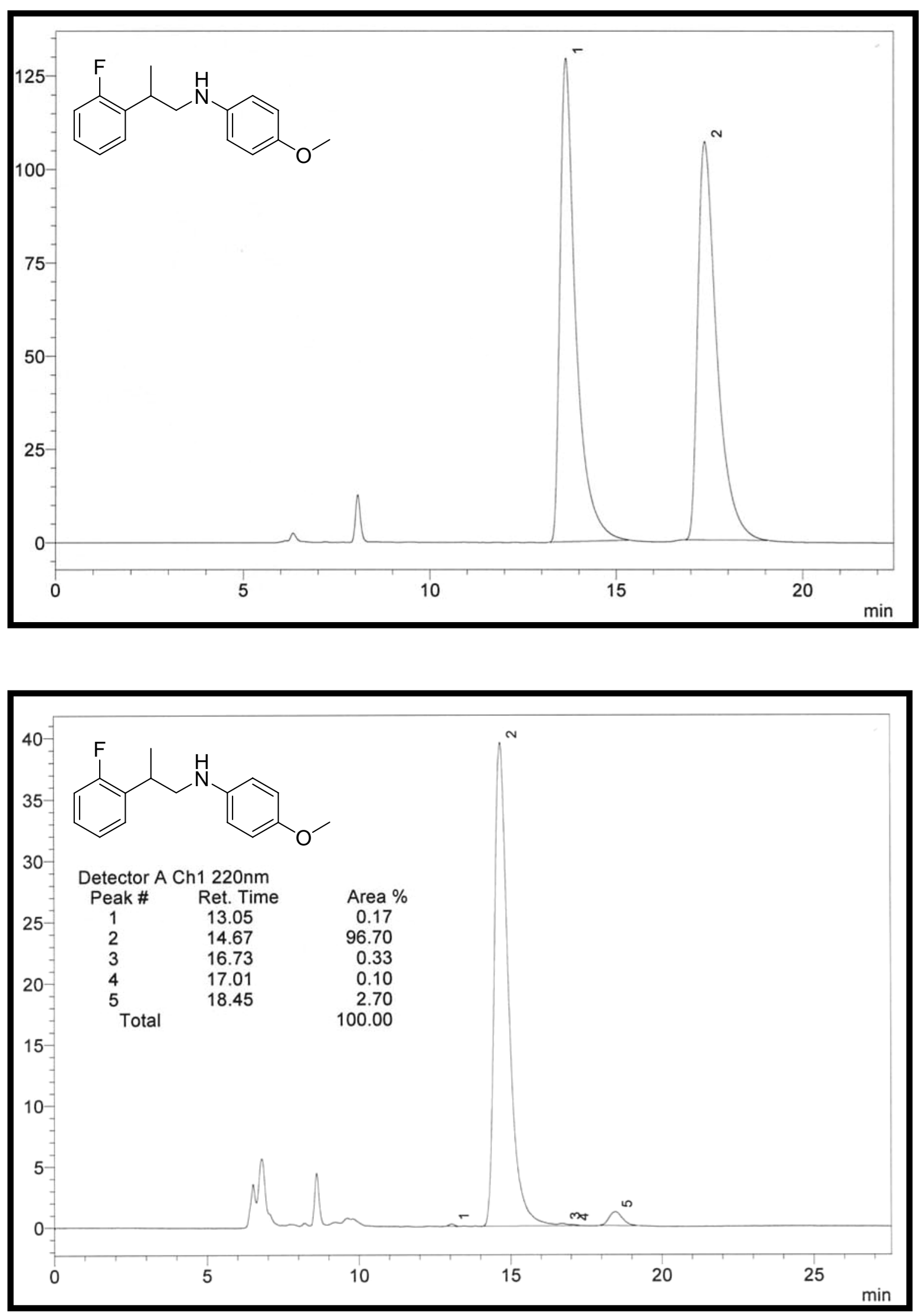

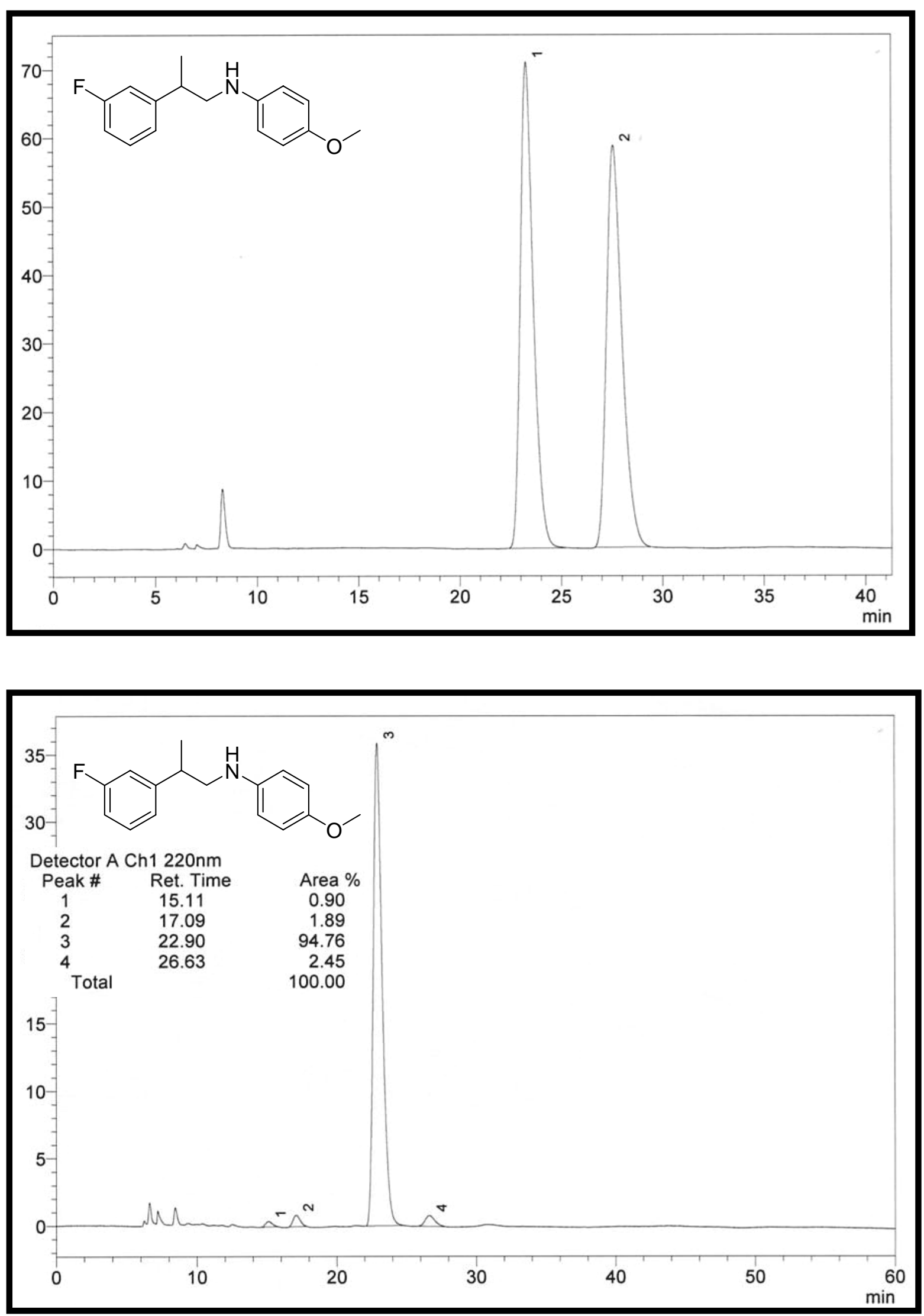

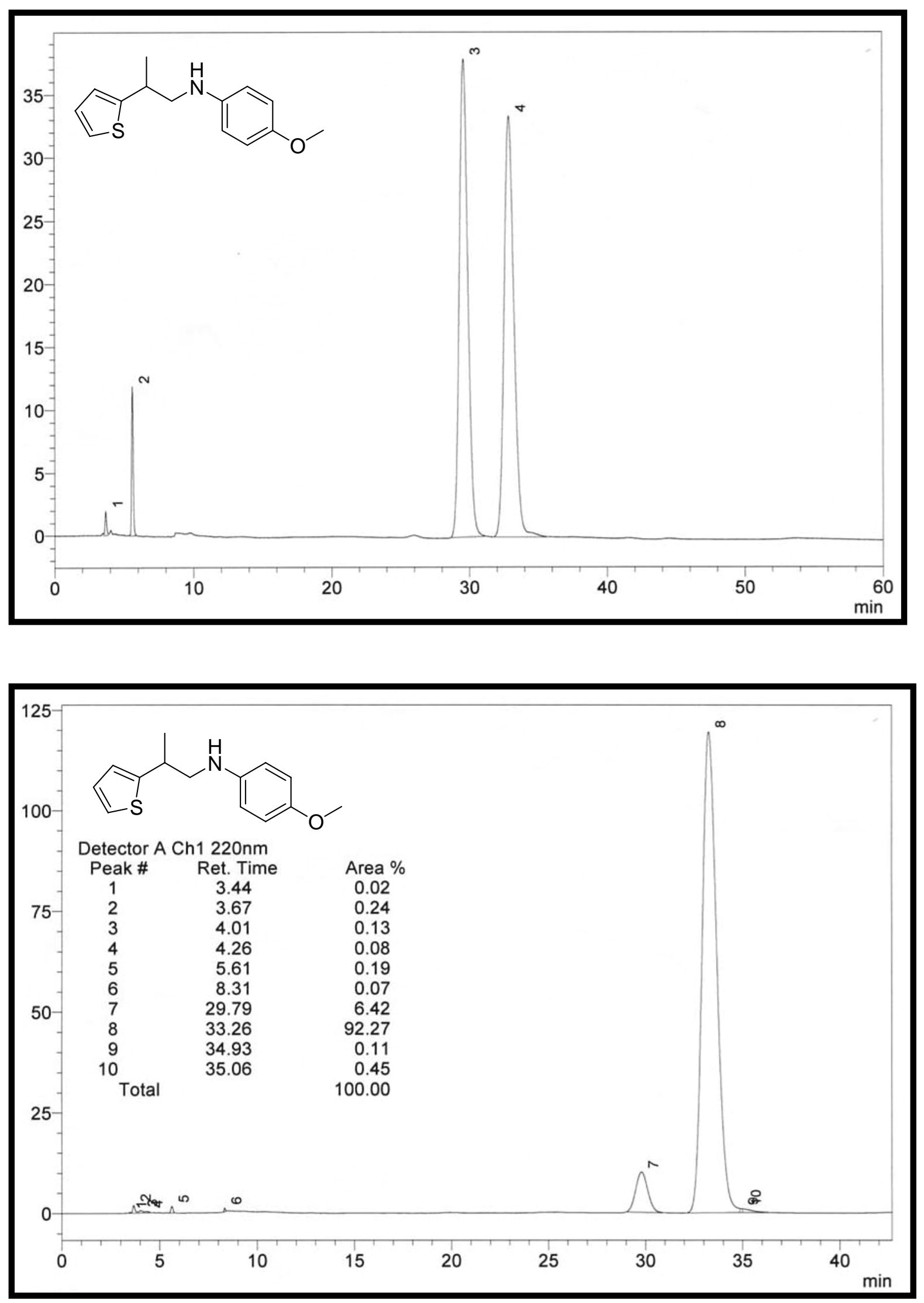

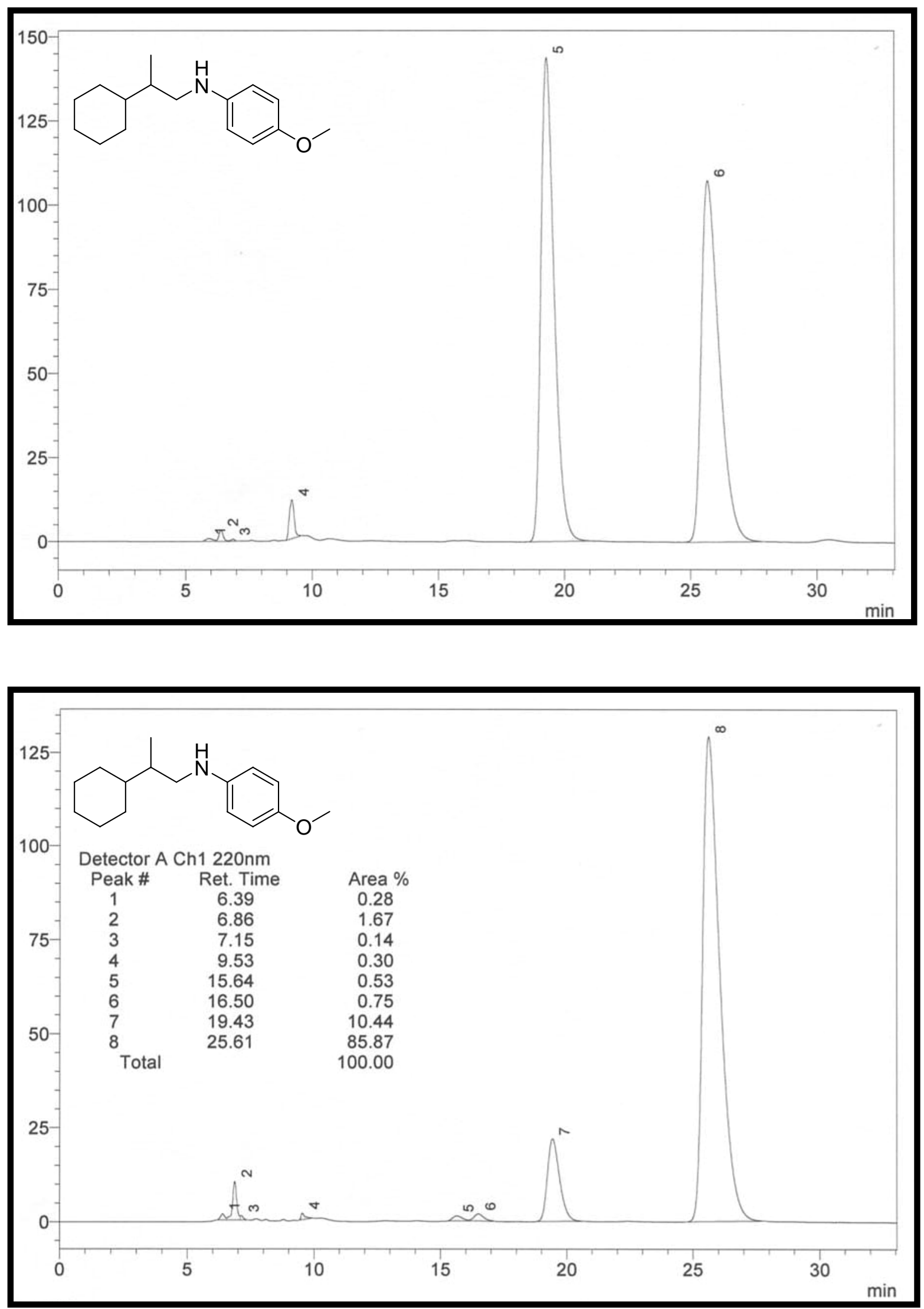

- S25 - 

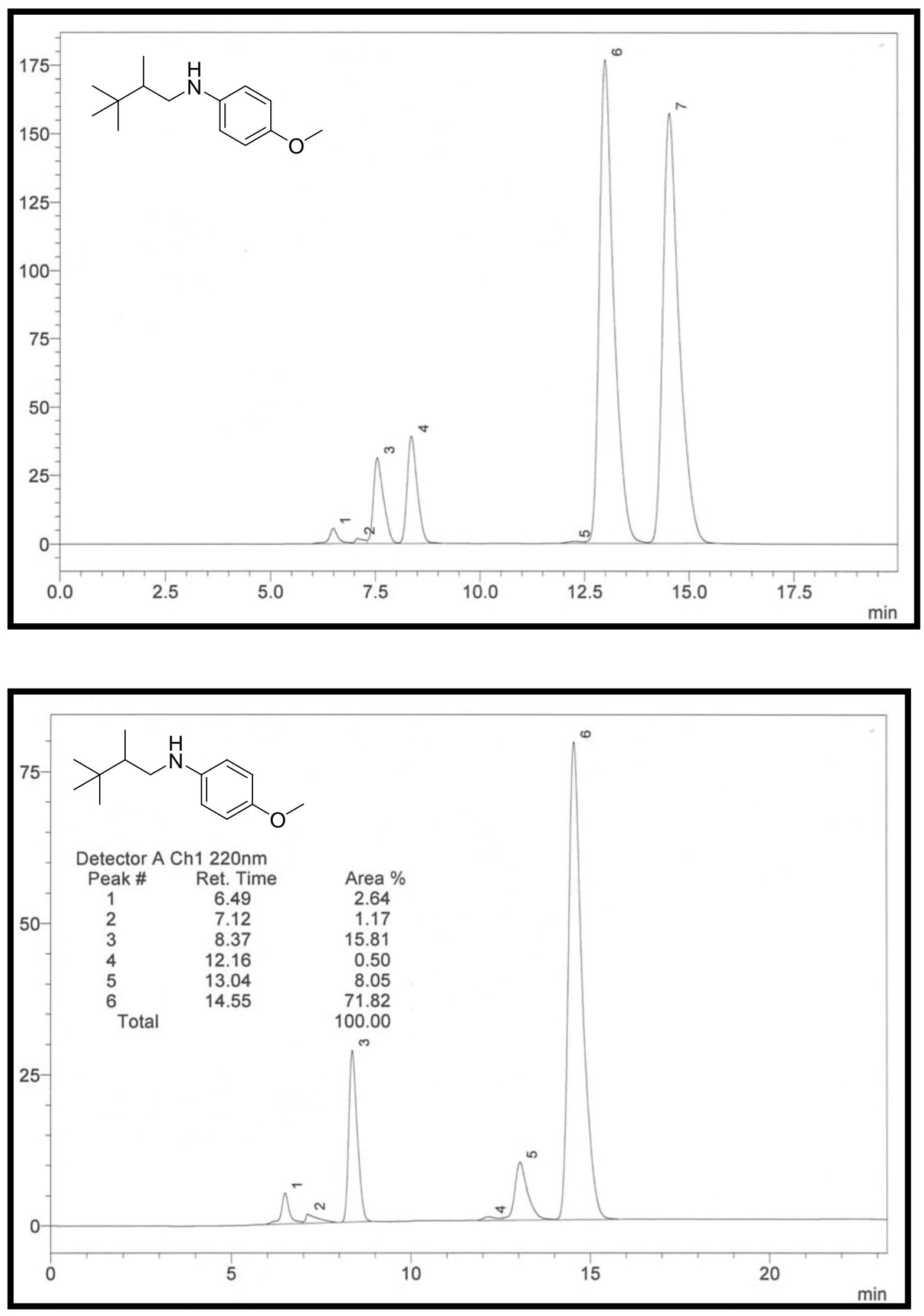

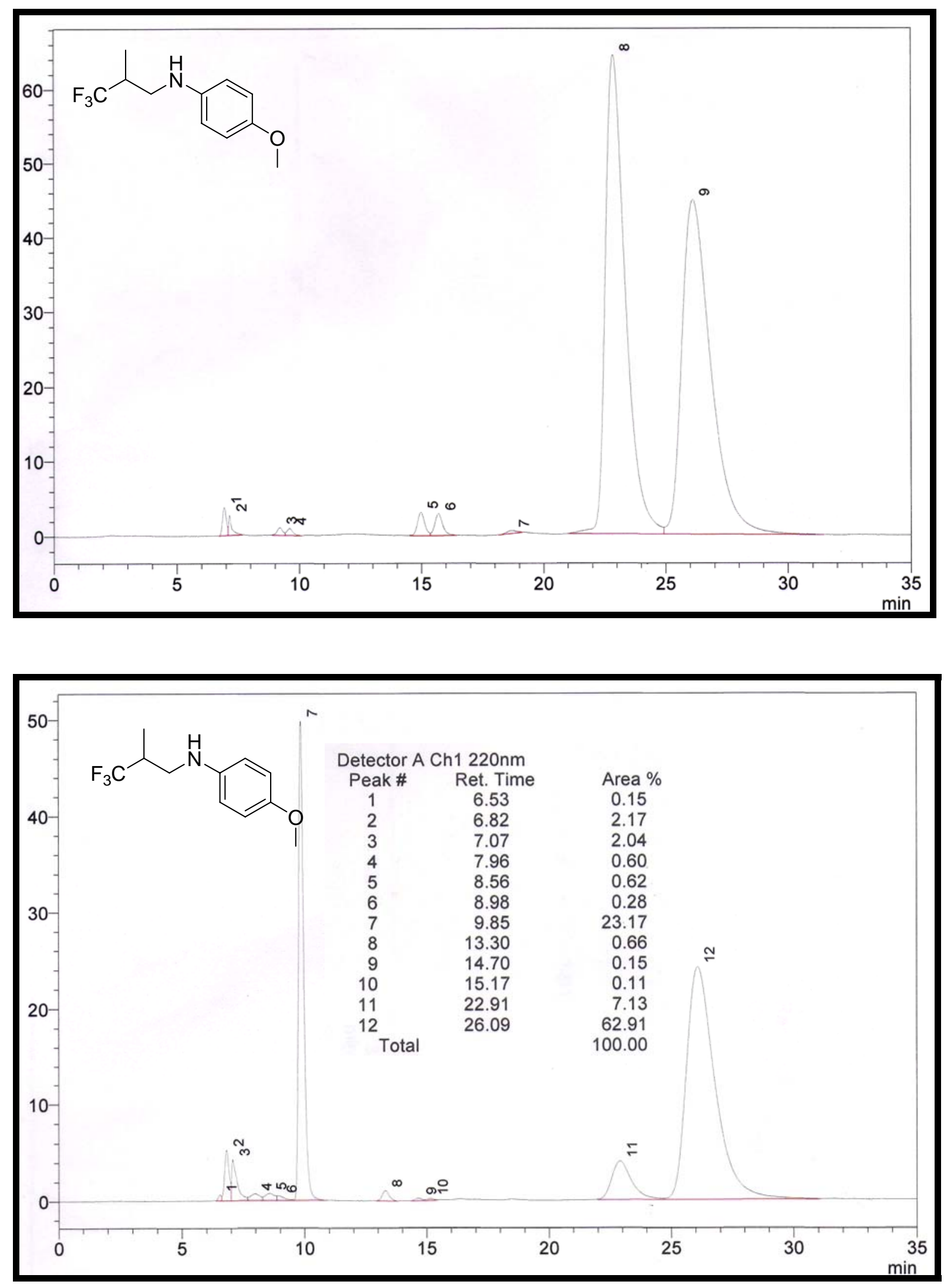

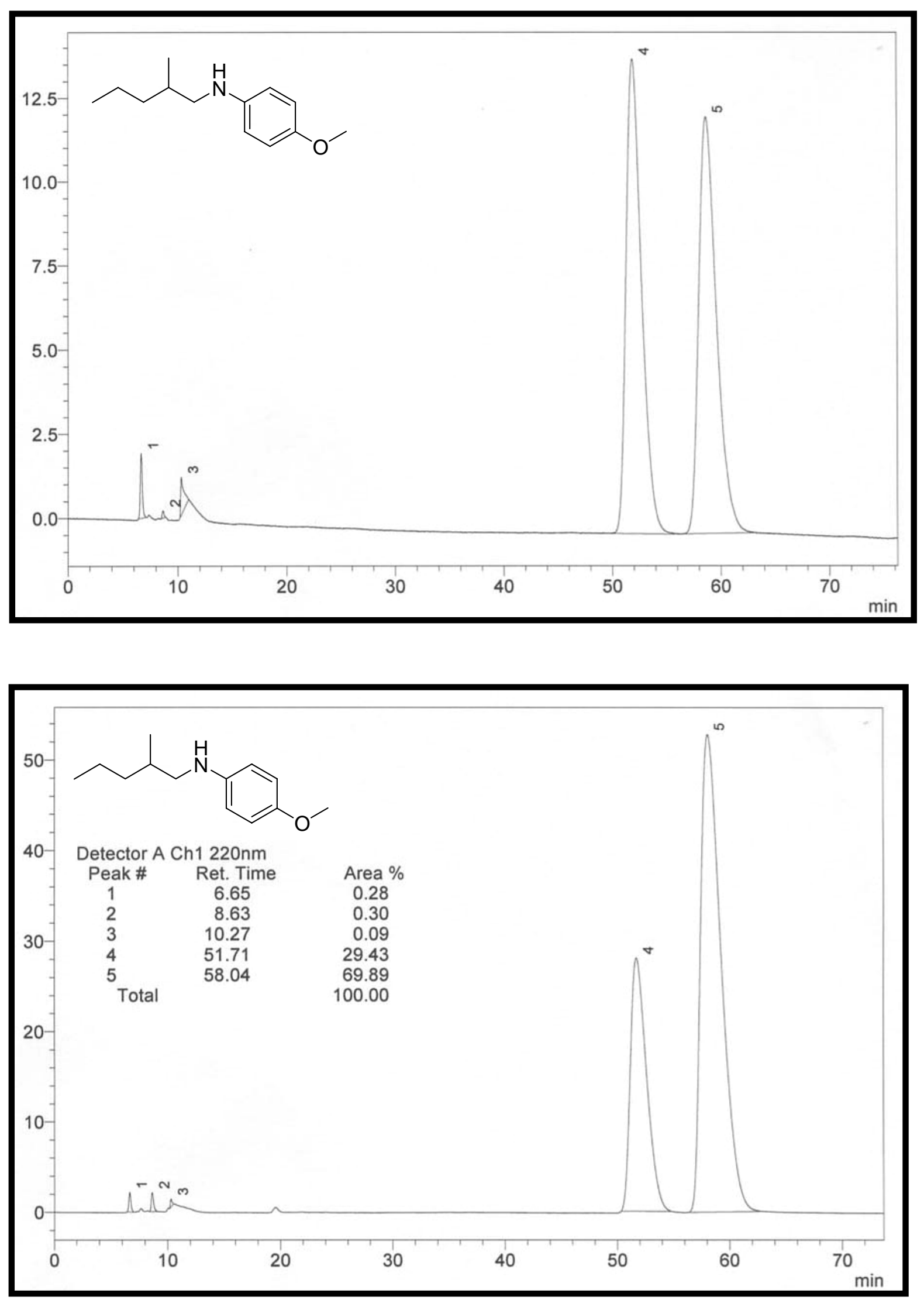

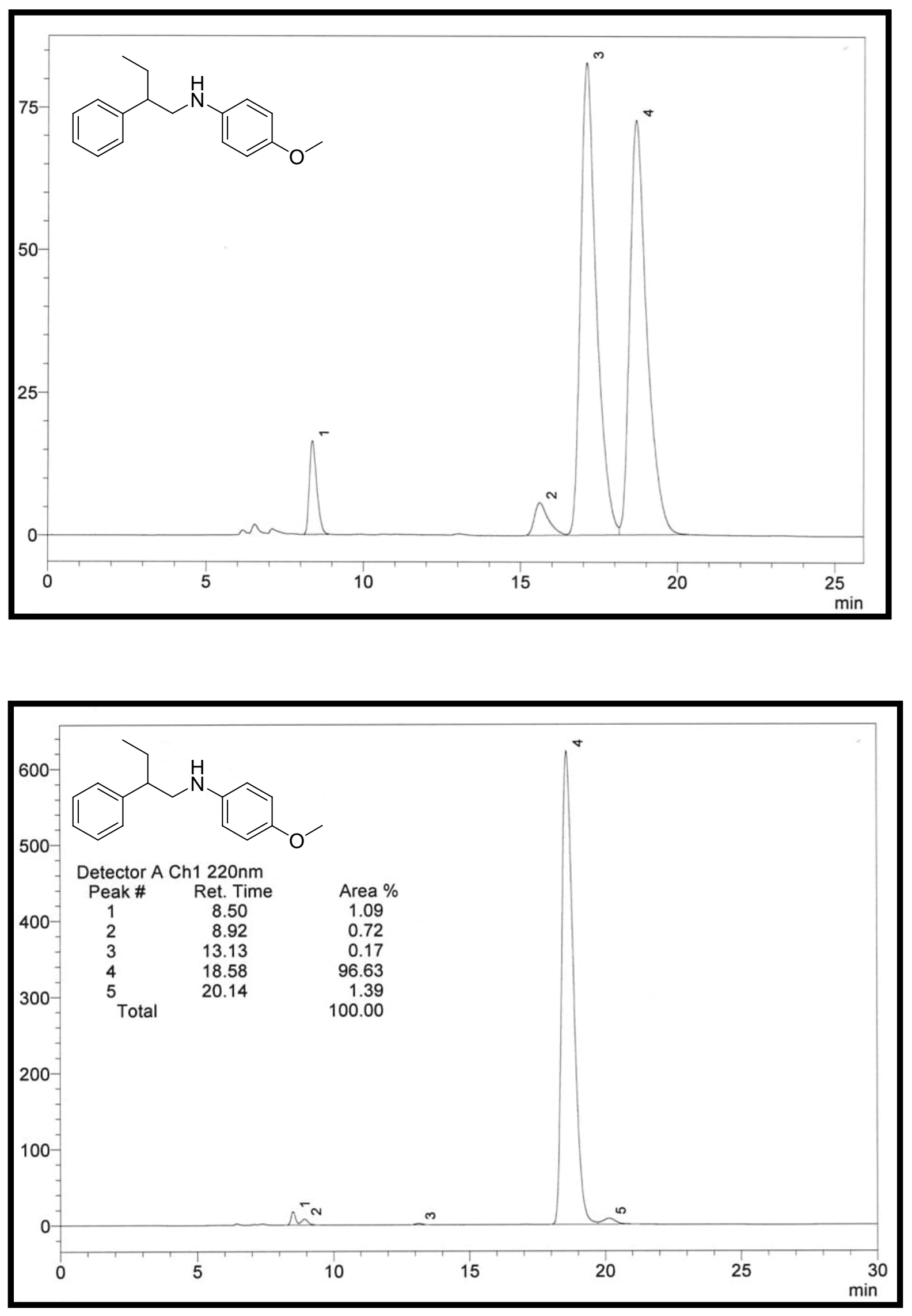

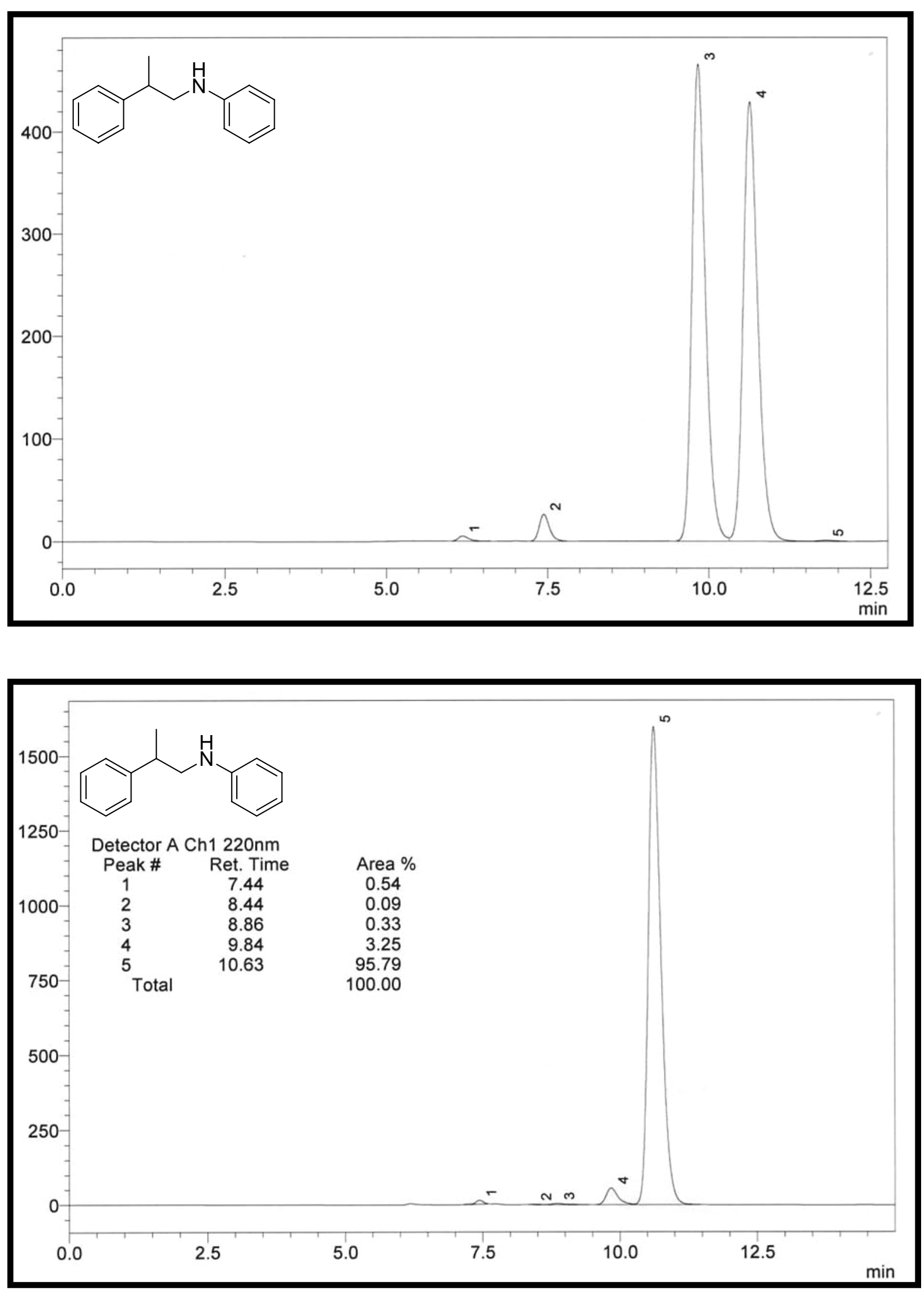

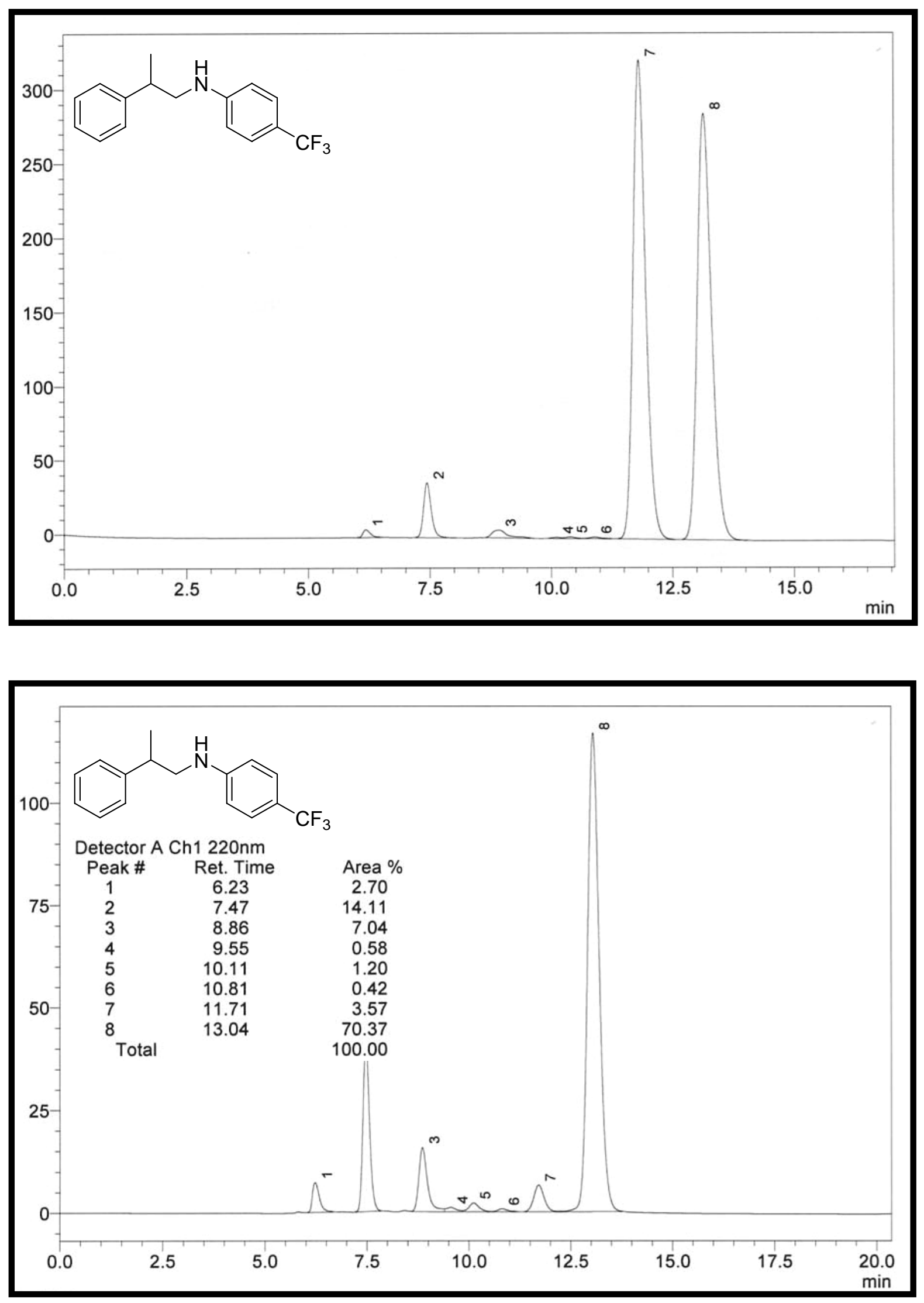

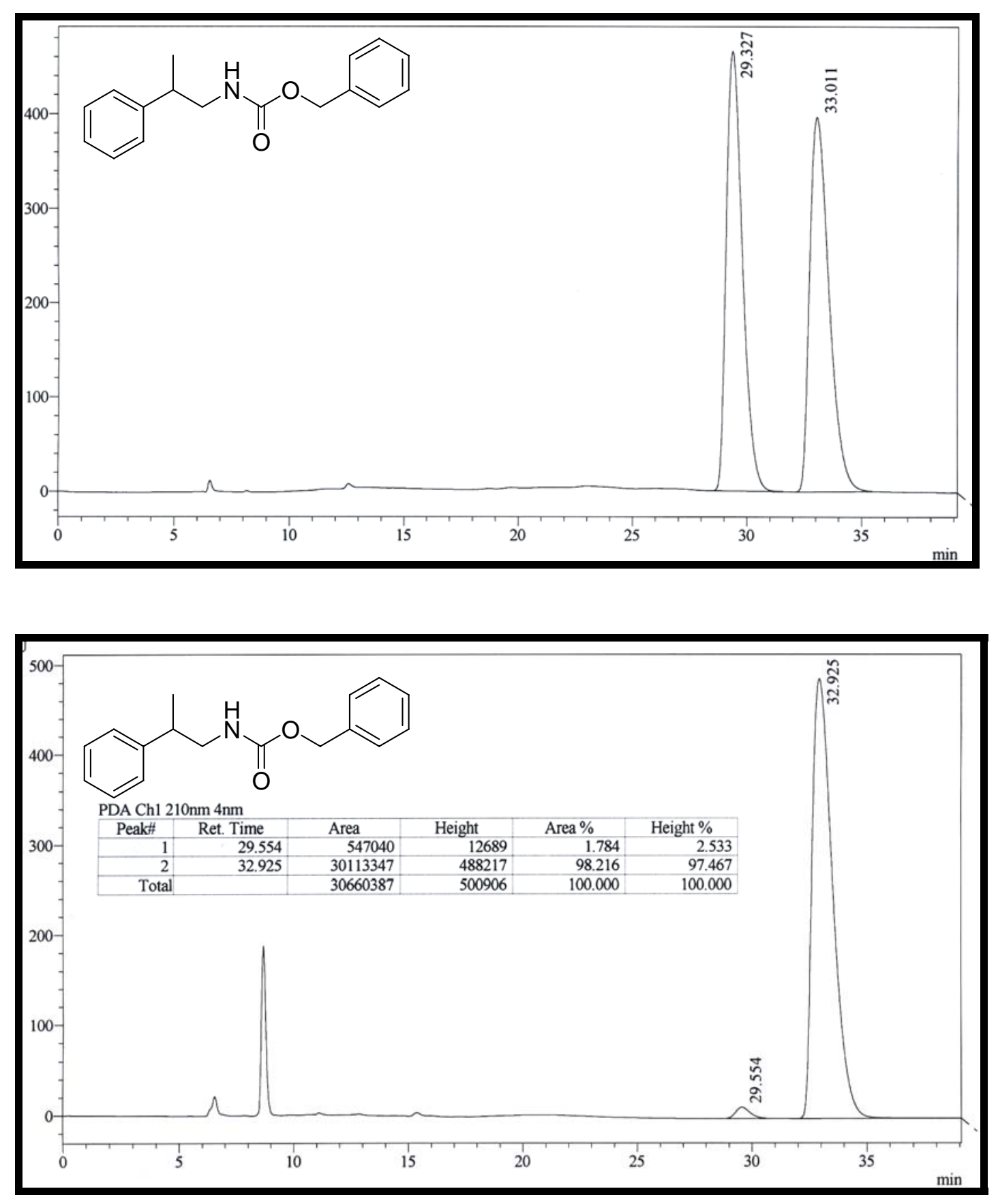

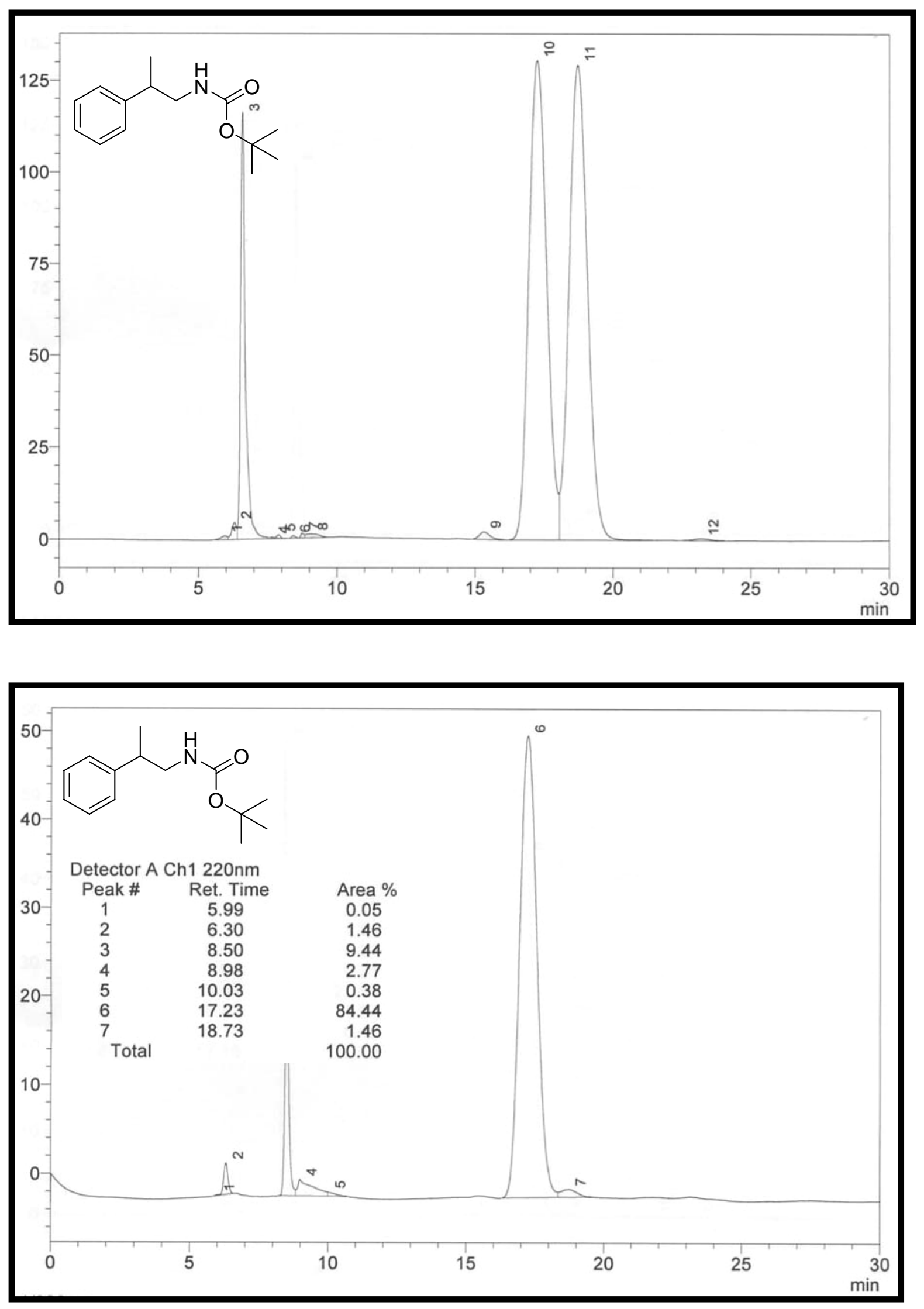

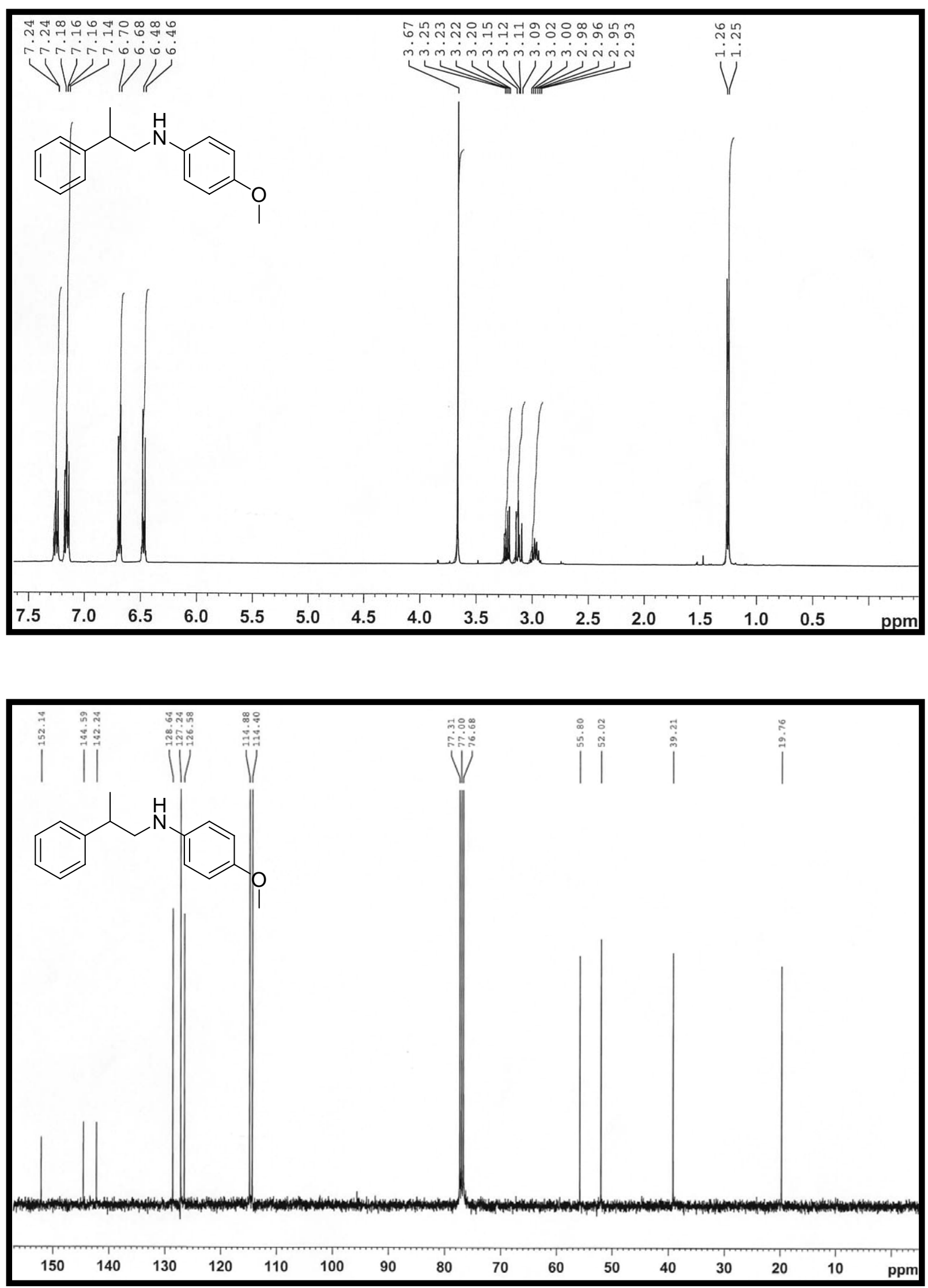

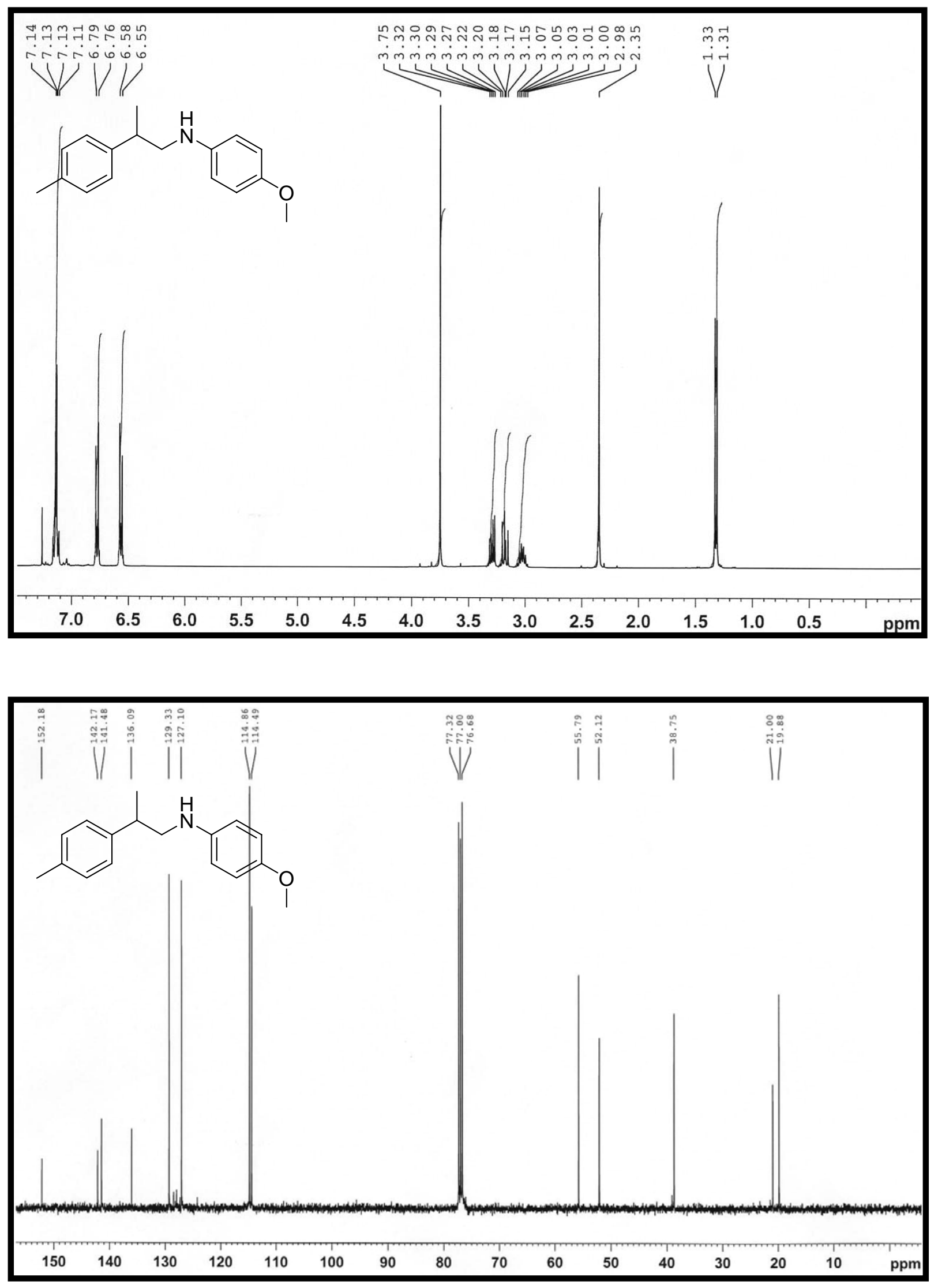

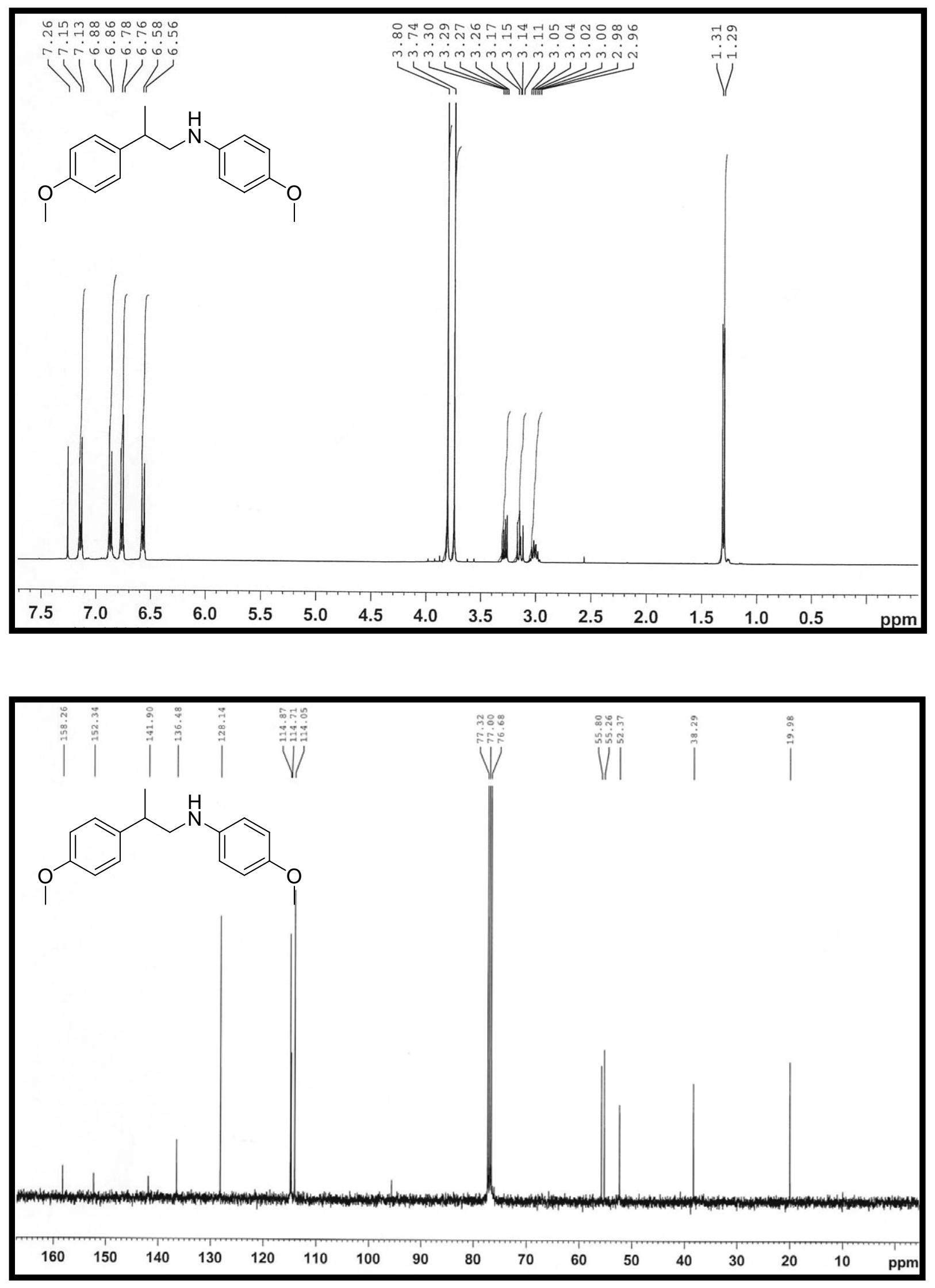

- S36 - 

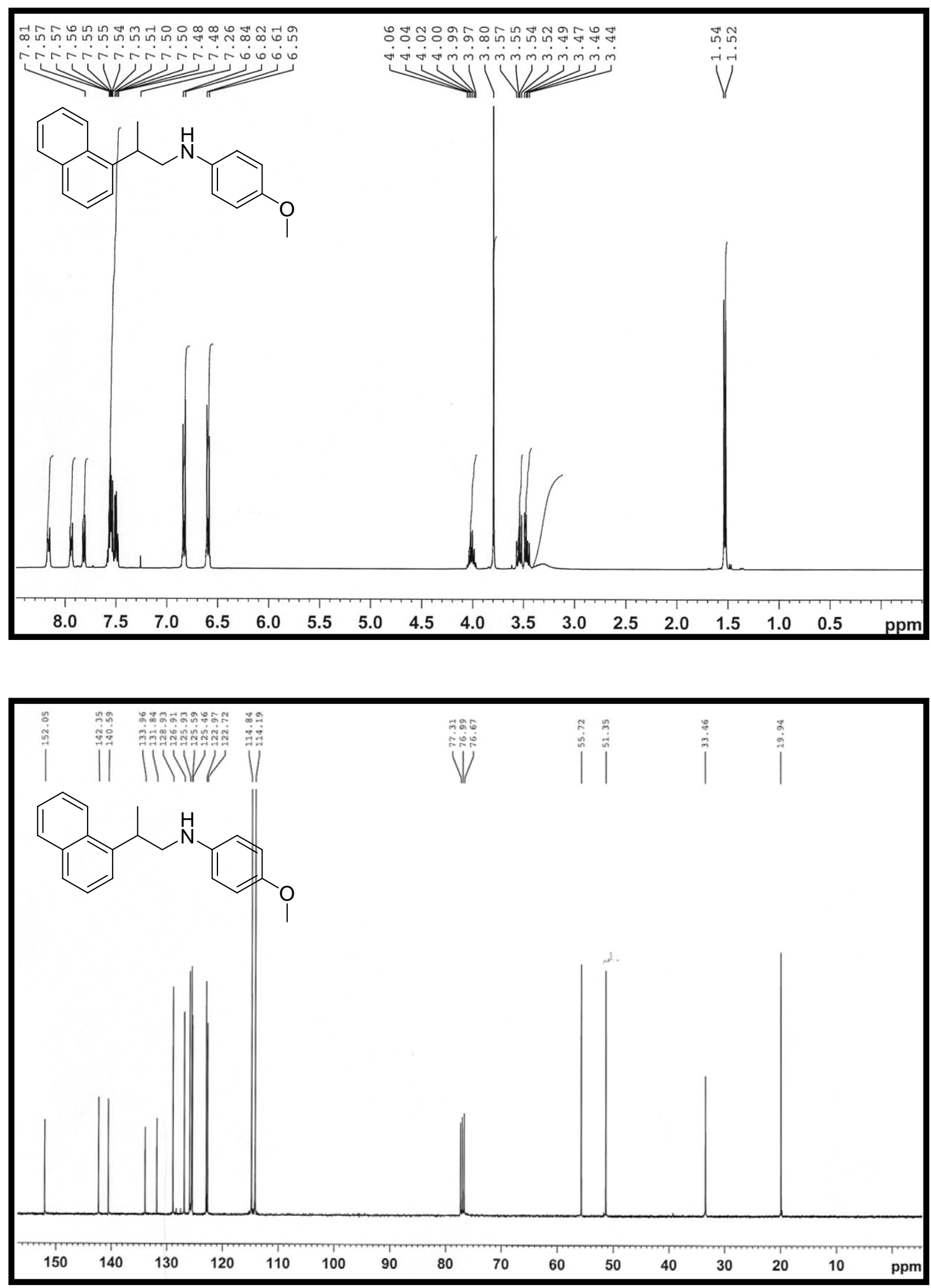

- S37 - 

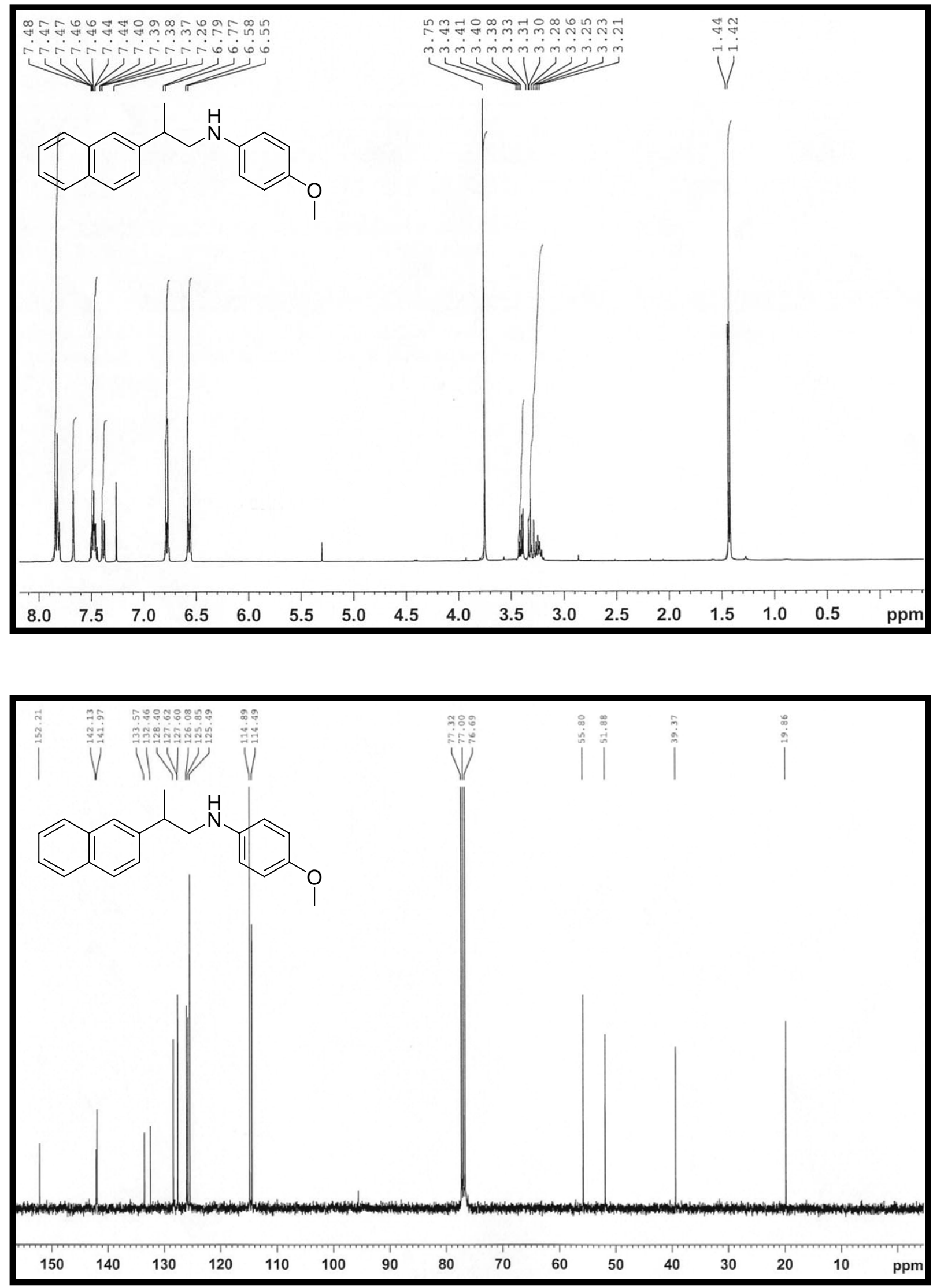

- S38 - 

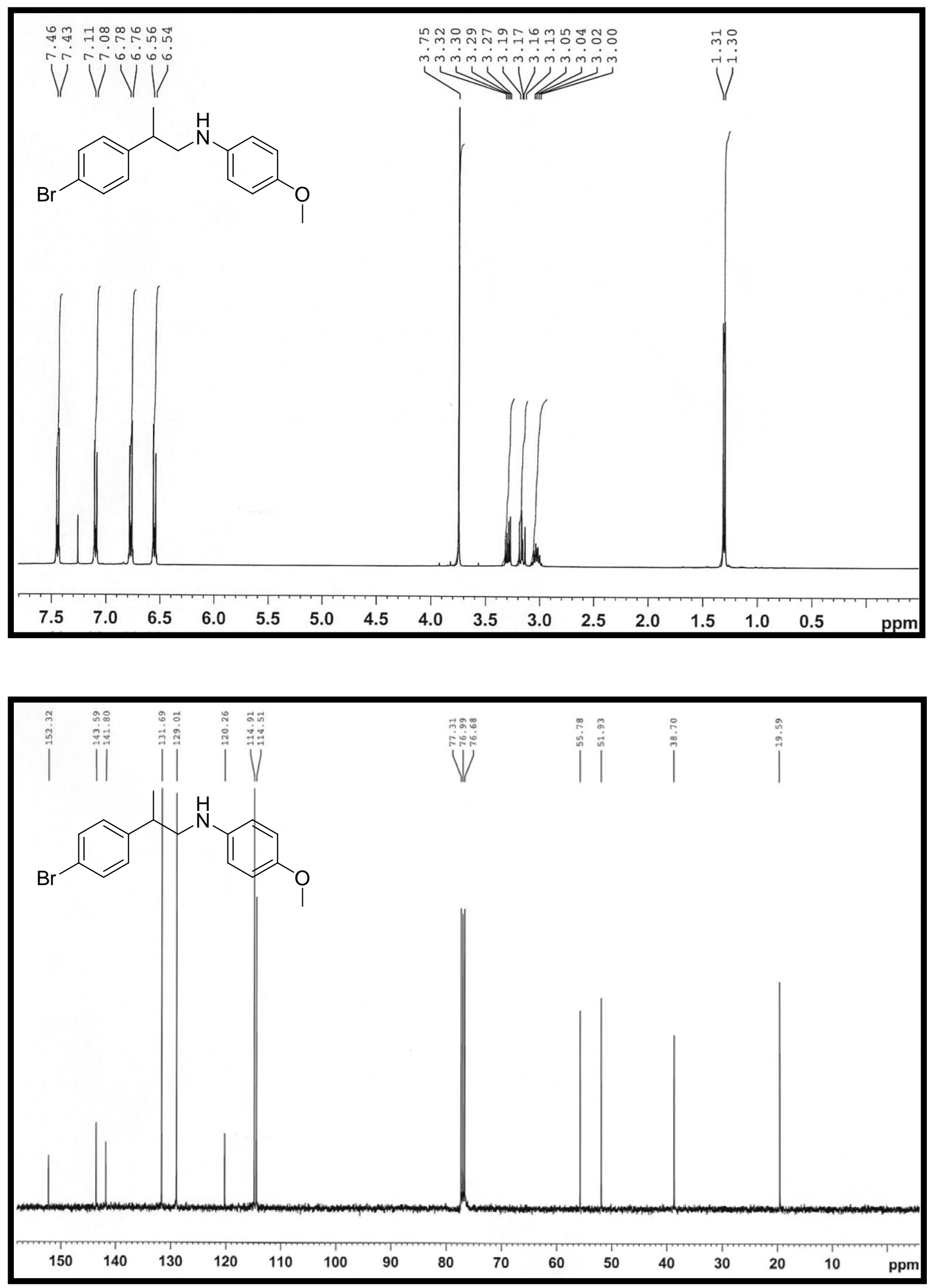

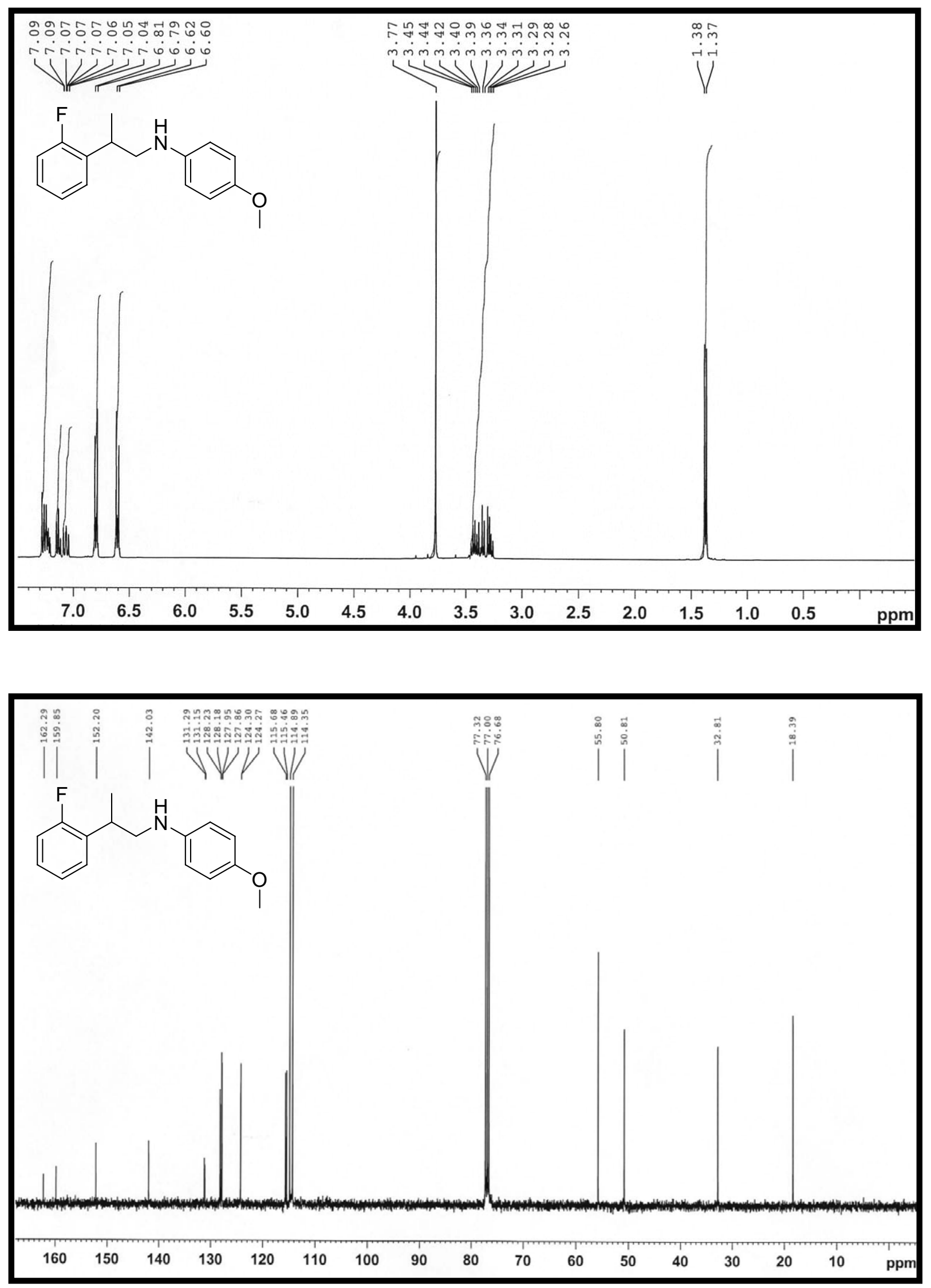

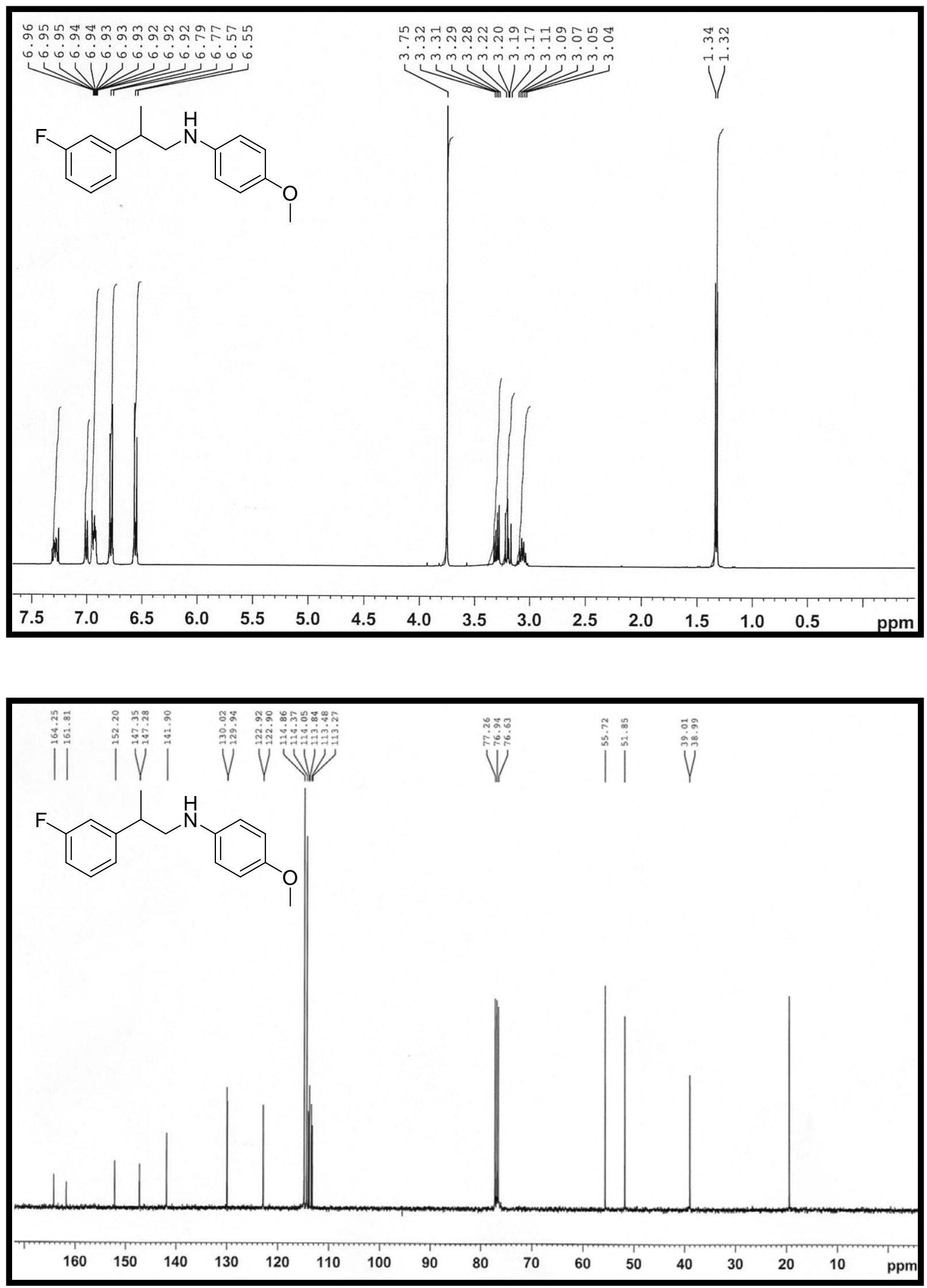

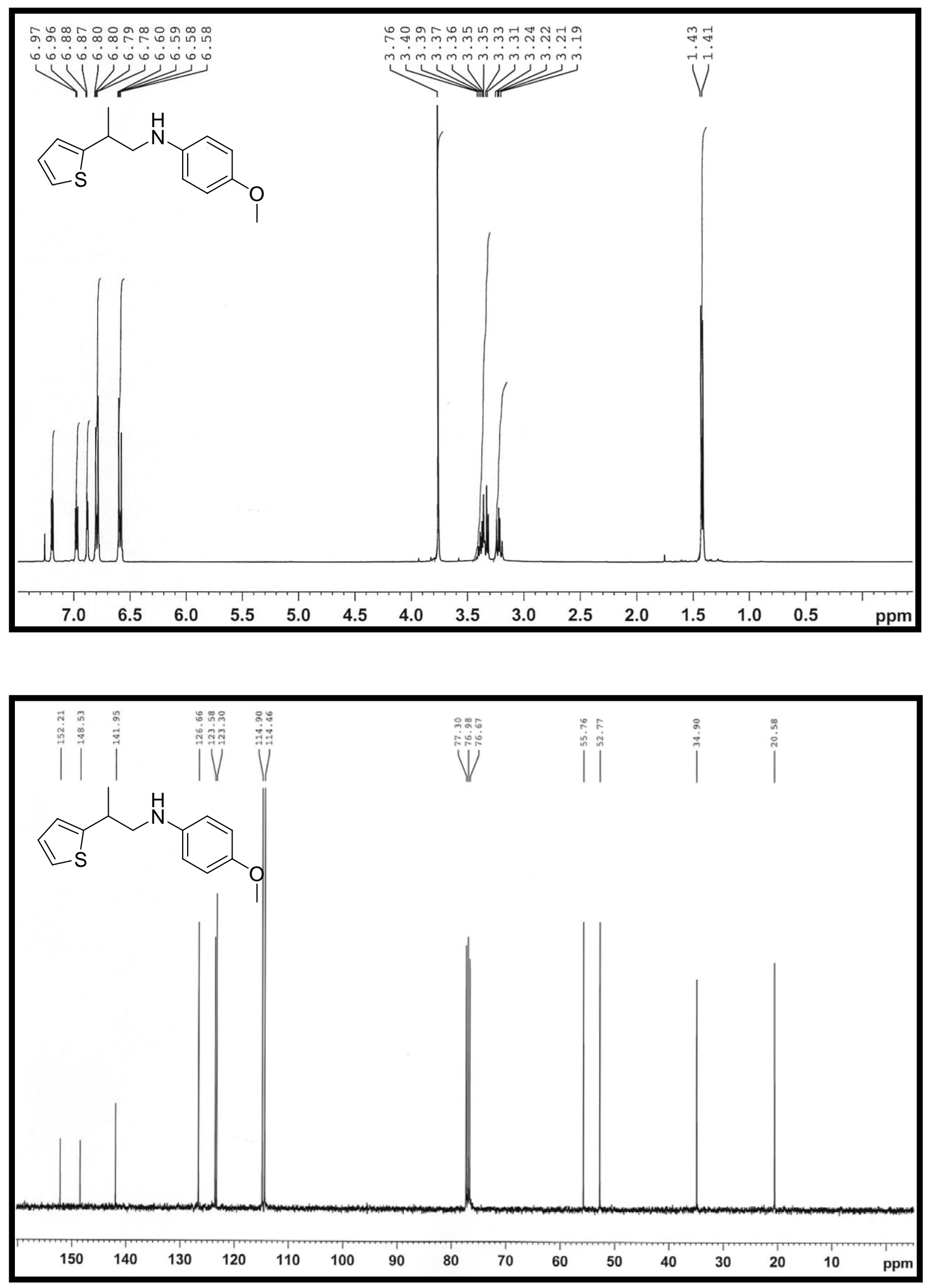

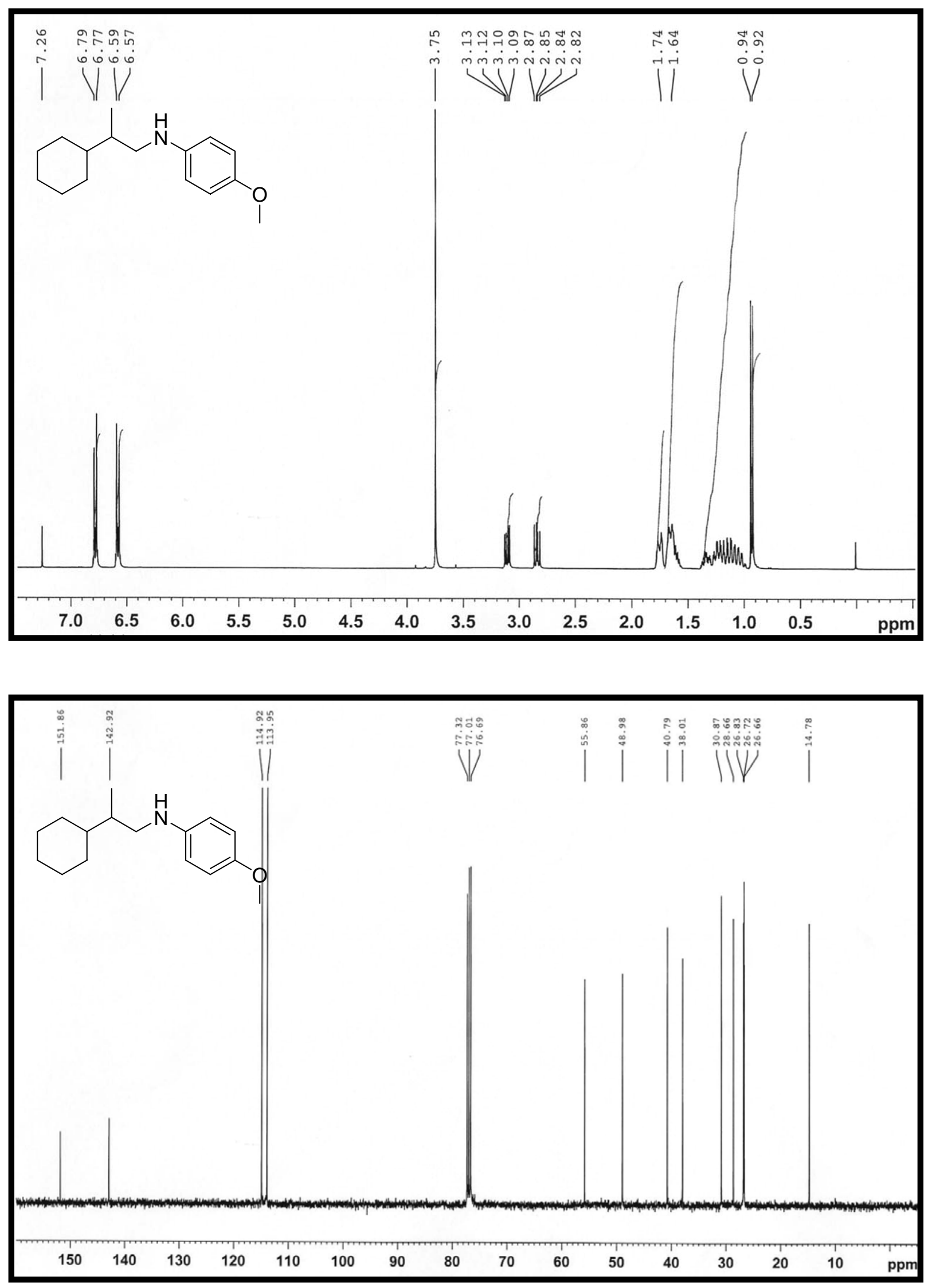

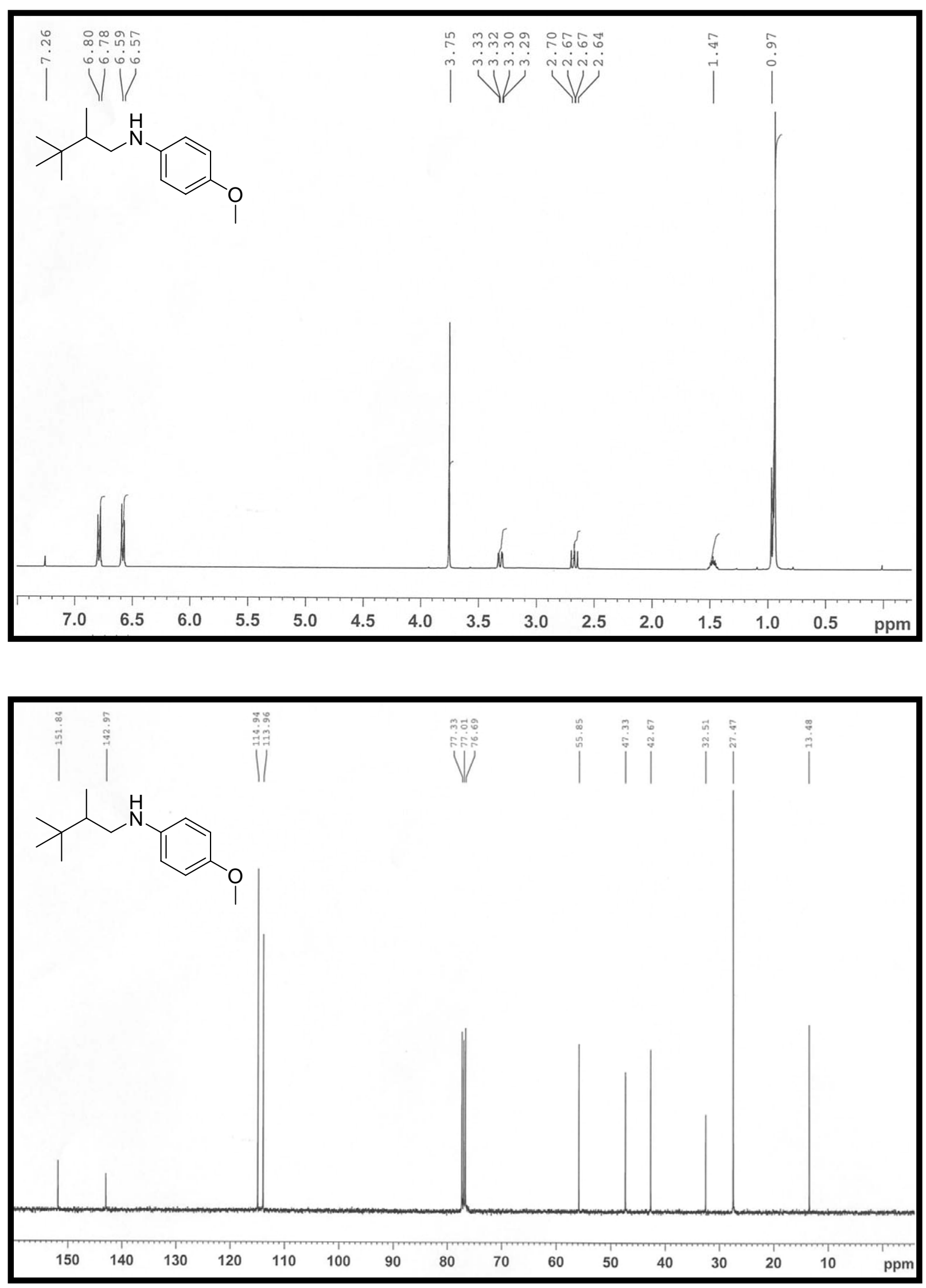

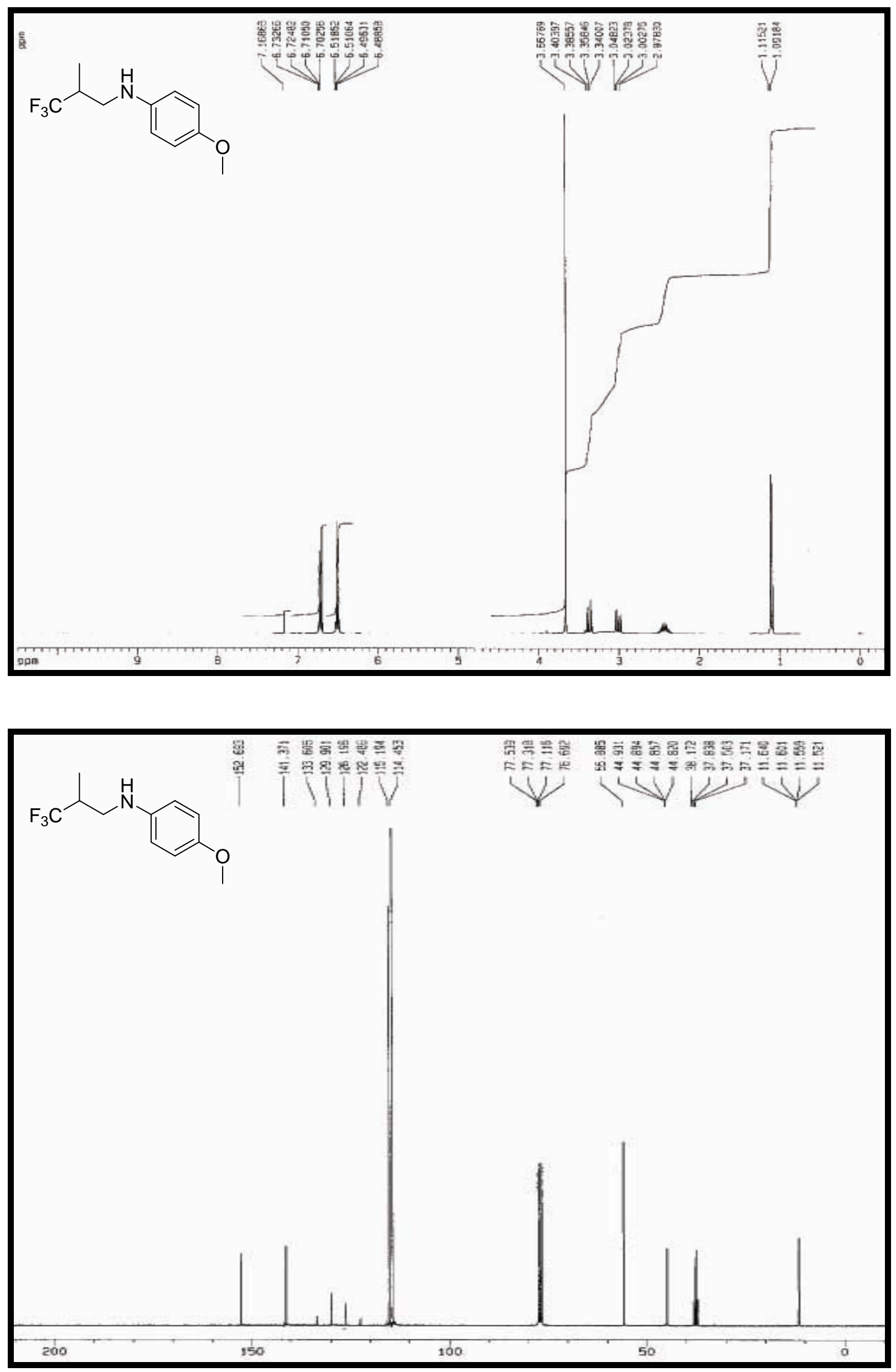

- S45 - 

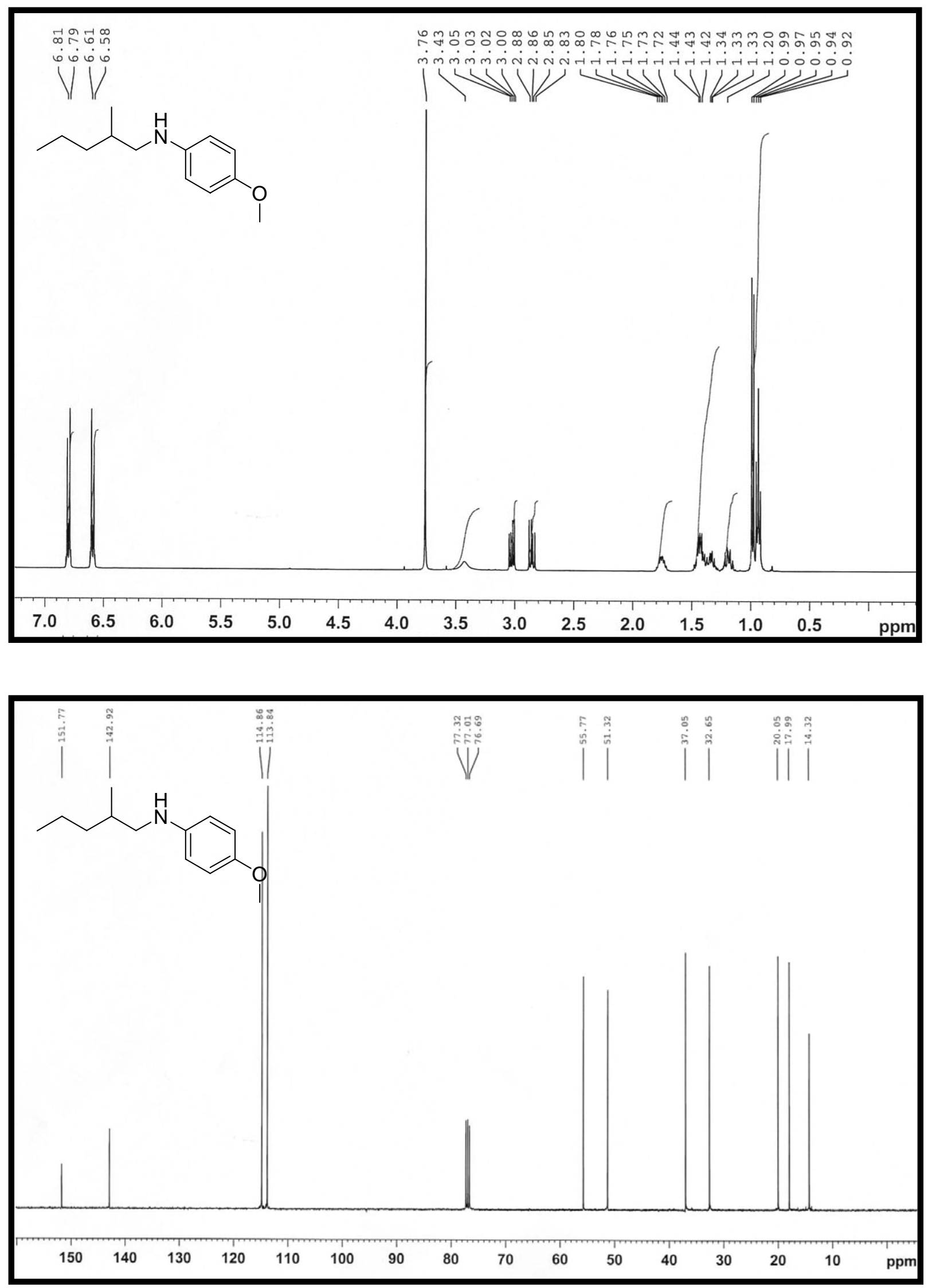

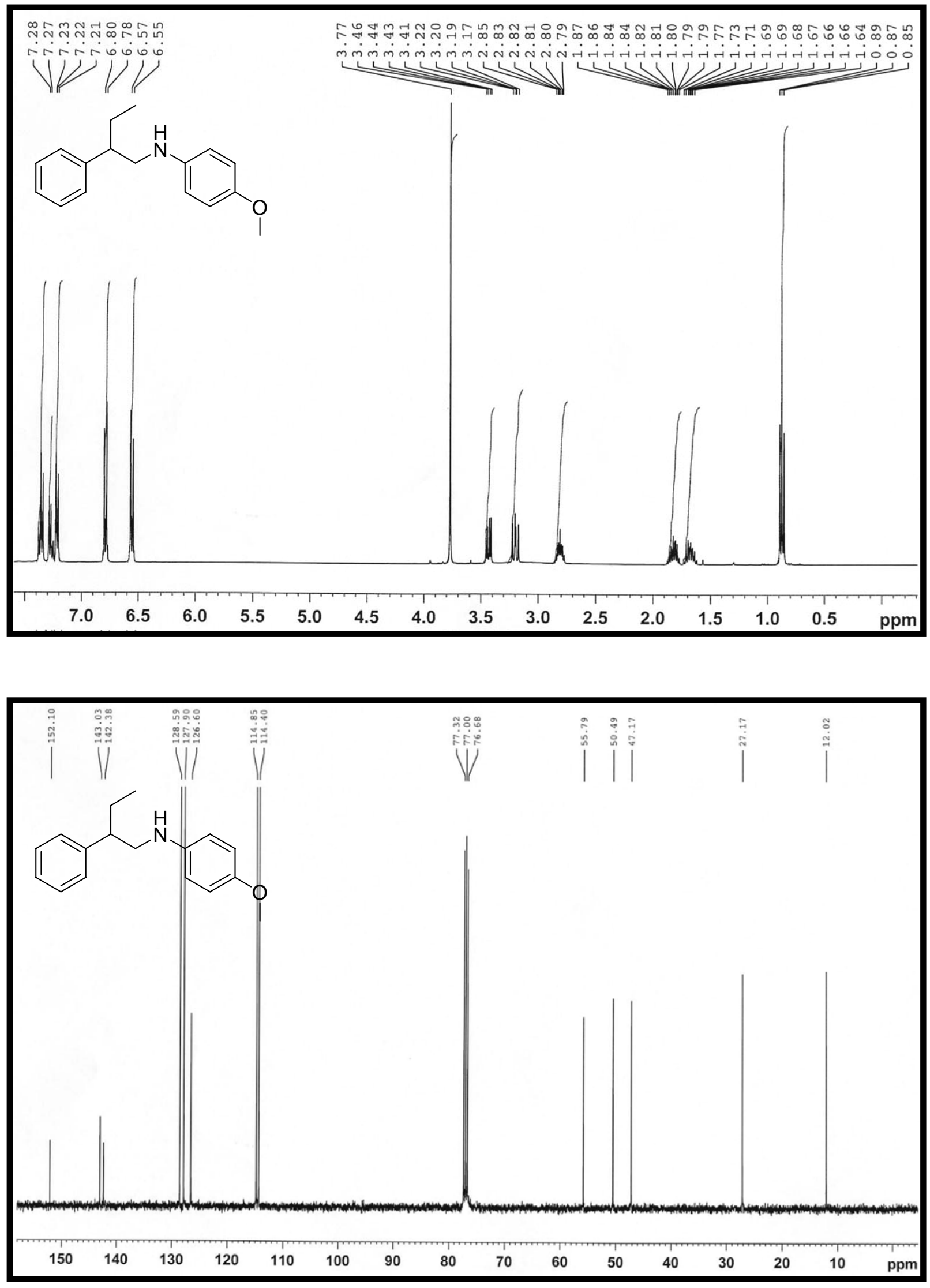

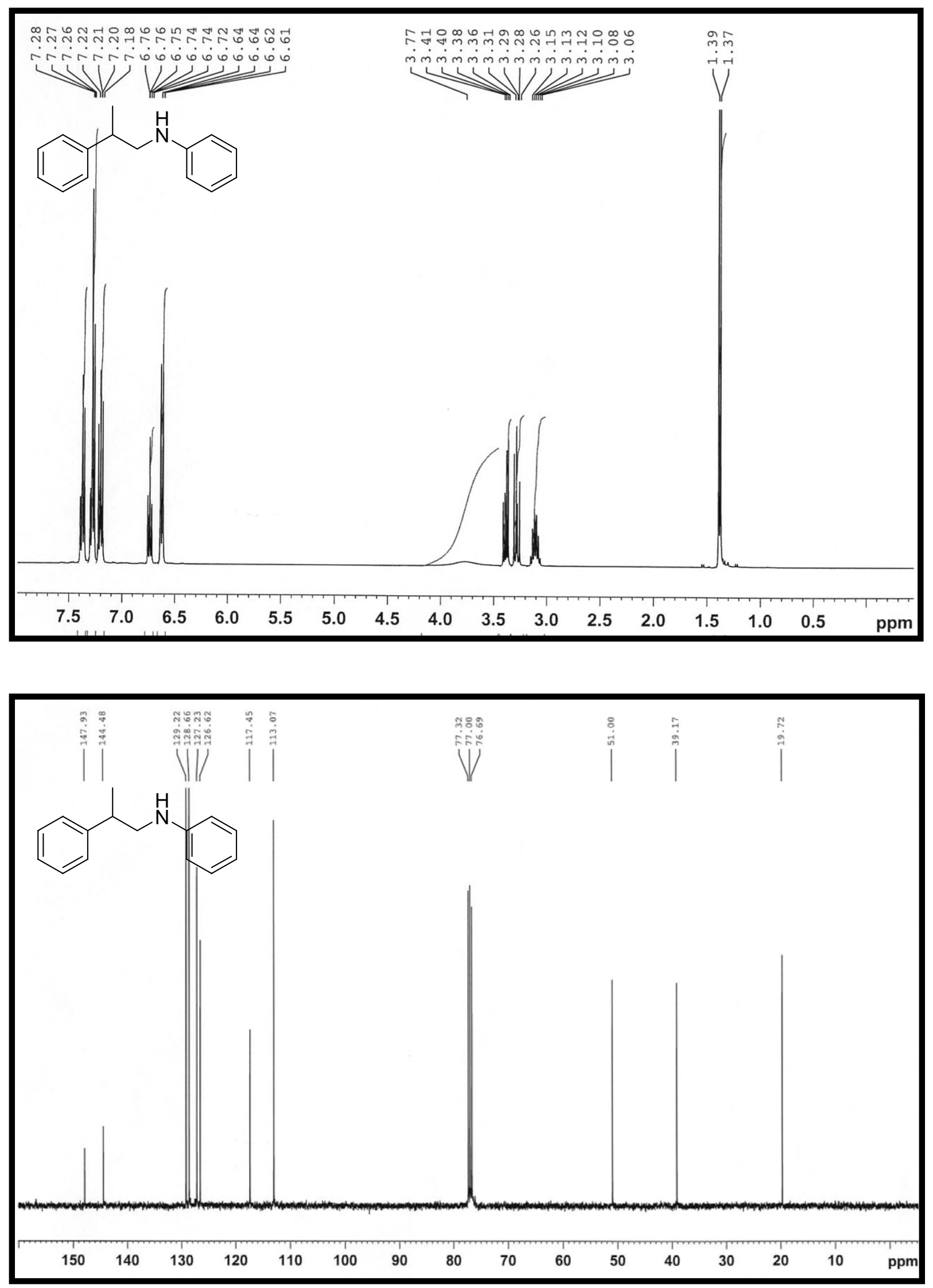

- S48 - 

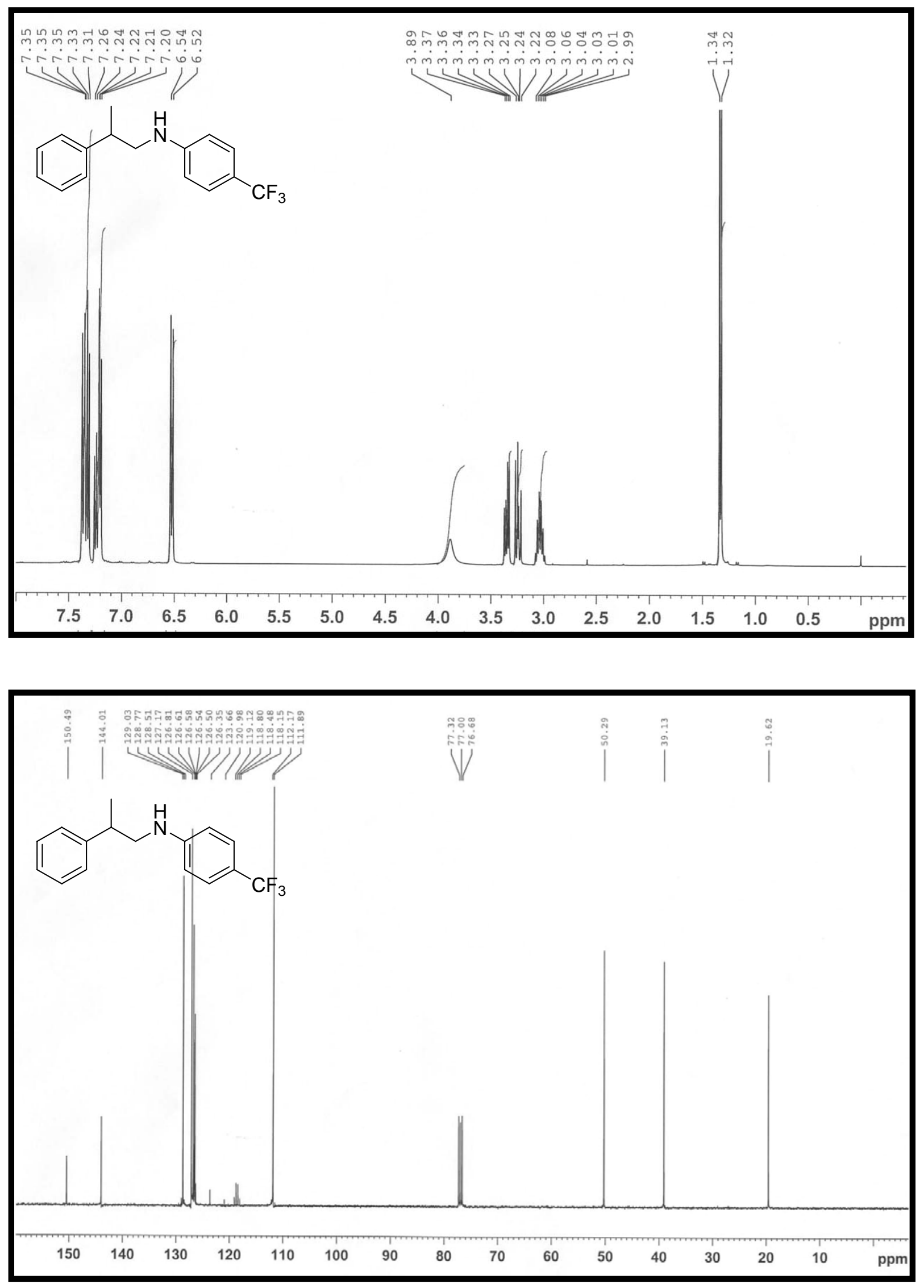


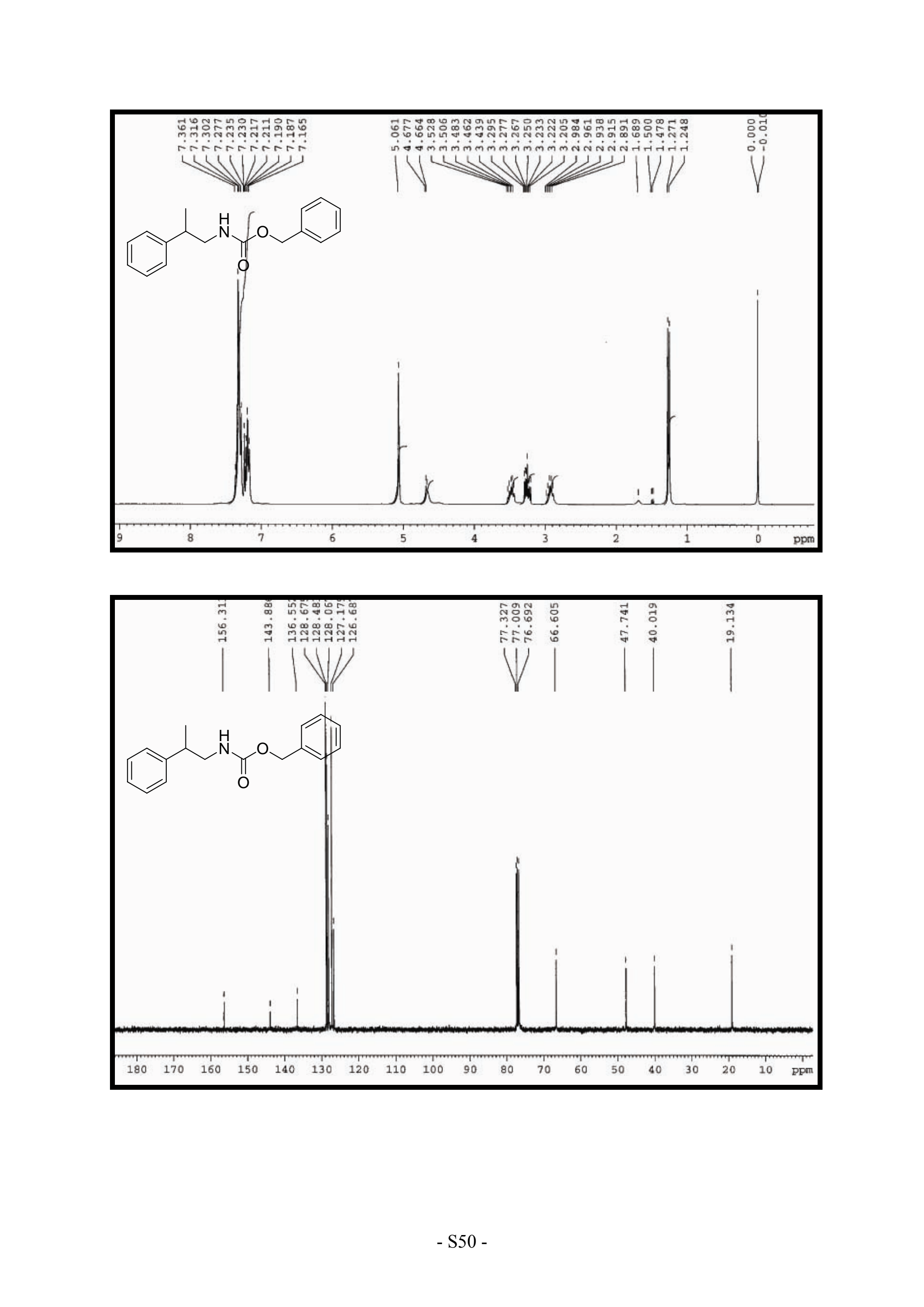



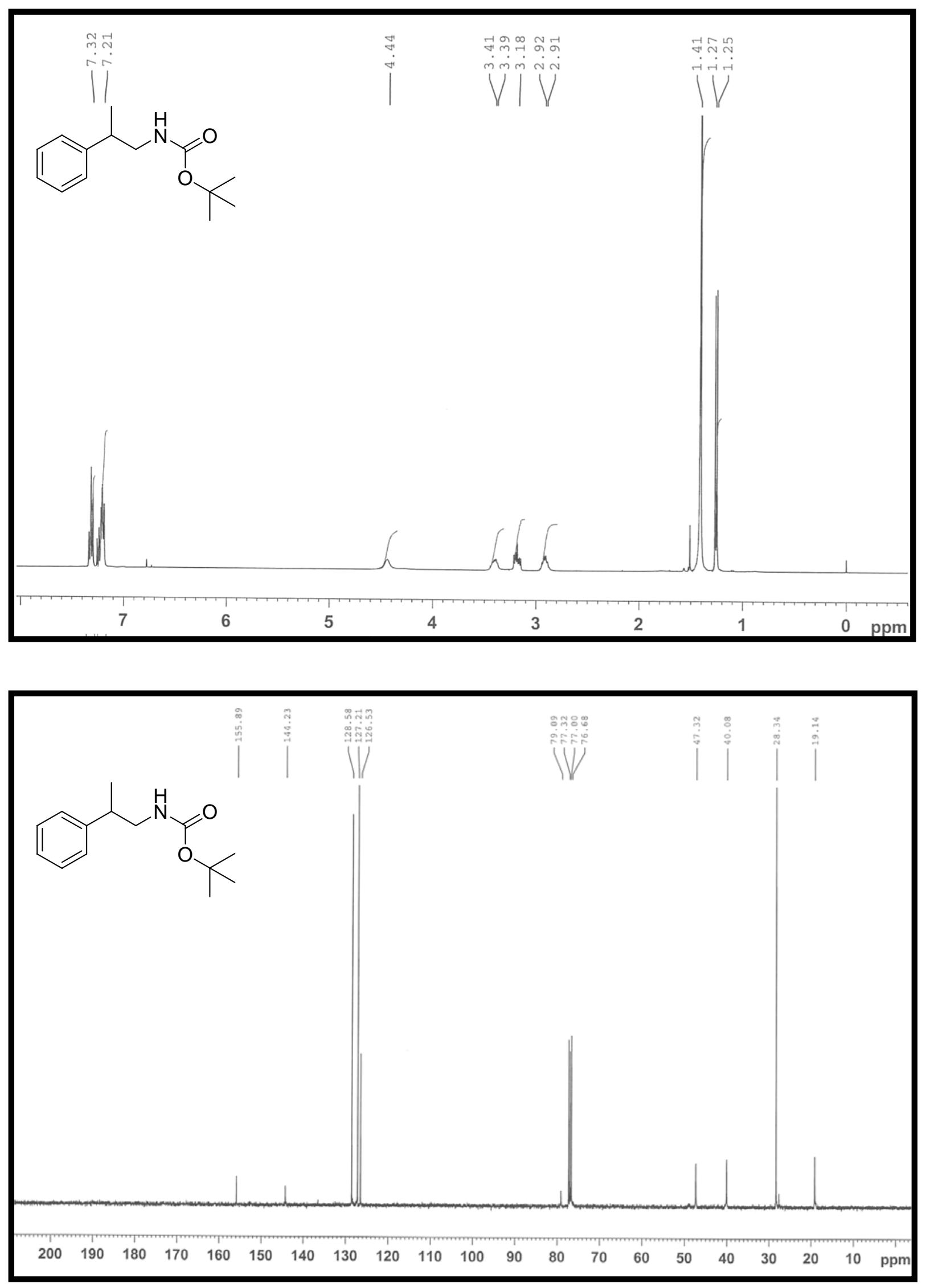

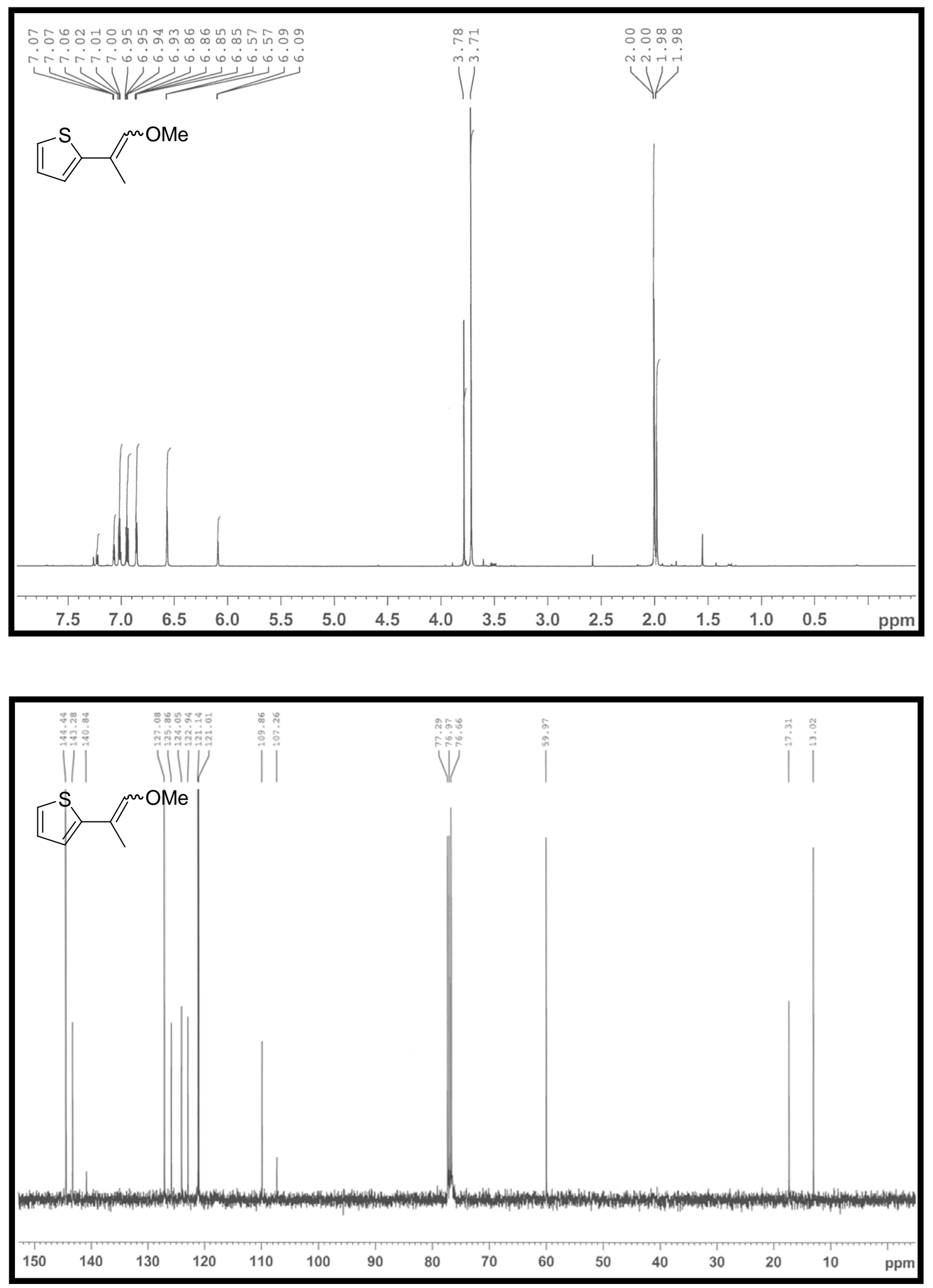